\title{
On the ability of RegCM4 regional climate model to simulate surface so- lar radiation patterns over Europe: an assessment using satellite-based observations
}

\author{
G. Alexandri et al. \\ Correspondence to: G. Alexandri (alexang@auth.gr)
}

The copyright of individual parts of the supplement might differ from the CC-BY 3.0 licence. 


\section{Statistical Metrics}

- Normalized standard deviation (NSD) expresses the amplitude of the variance in modeled values versus the observed values:

$$
N S D=\frac{\sigma_{\operatorname{Re} g C M}}{\sigma_{C M S A F}}
$$

where $\sigma_{\mathrm{RegCM}}$ and $\sigma_{\mathrm{CMSAF}}$ are the standard deviations of RegCM4 and CM SAF respectively.

- Modified Normalized Mean Bias (MNMB) is a normalization based on the observed (CM $\mathrm{SAF})$ and the modeled (RegCM) value and is limited between -2 and $+2(-2$ expresses underestimation $-200 \%$ and +2 expresses overestimation $+200 \%)$ :

$$
M N M B=\frac{2}{N} \sum_{i=1}^{N} \frac{\operatorname{Re} g C M_{i}-C M S A F_{i}}{\operatorname{Re} g C M_{i}+C M S A F_{i}}
$$

- RMSE expresses the standard deviation of the differences between two timeseries

(RegCM and CM SAF):

$$
R M S E=\sqrt{\frac{1}{n} \sum_{i=1}^{n}\left(\operatorname{Re} g C M_{i}-C M S A F_{i}\right)^{2}}
$$




\section{Surface Solar Radiation}

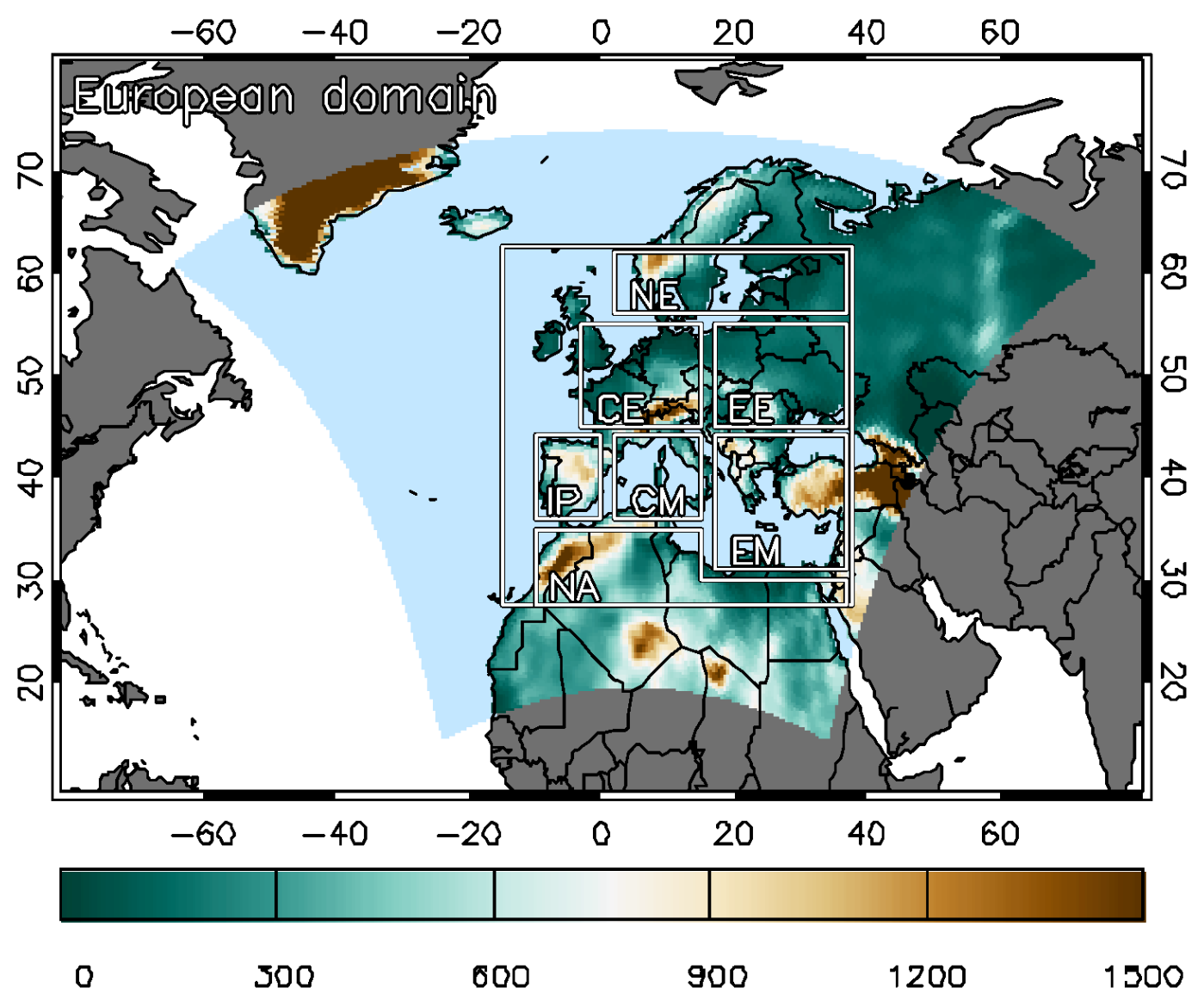

Figure S1. Topography of the RegCM4 simulation domain with the European box region and the 7 sub-regions used for the generalization of the results: Northern Europe (NE), Central Europe (CE), Eastern Europe (EE), Iberian Peninsula (IP), Central Mediterranean (CM), Eastern Mediterranean (EM) and Northern Africa (NA). 
a)

b)
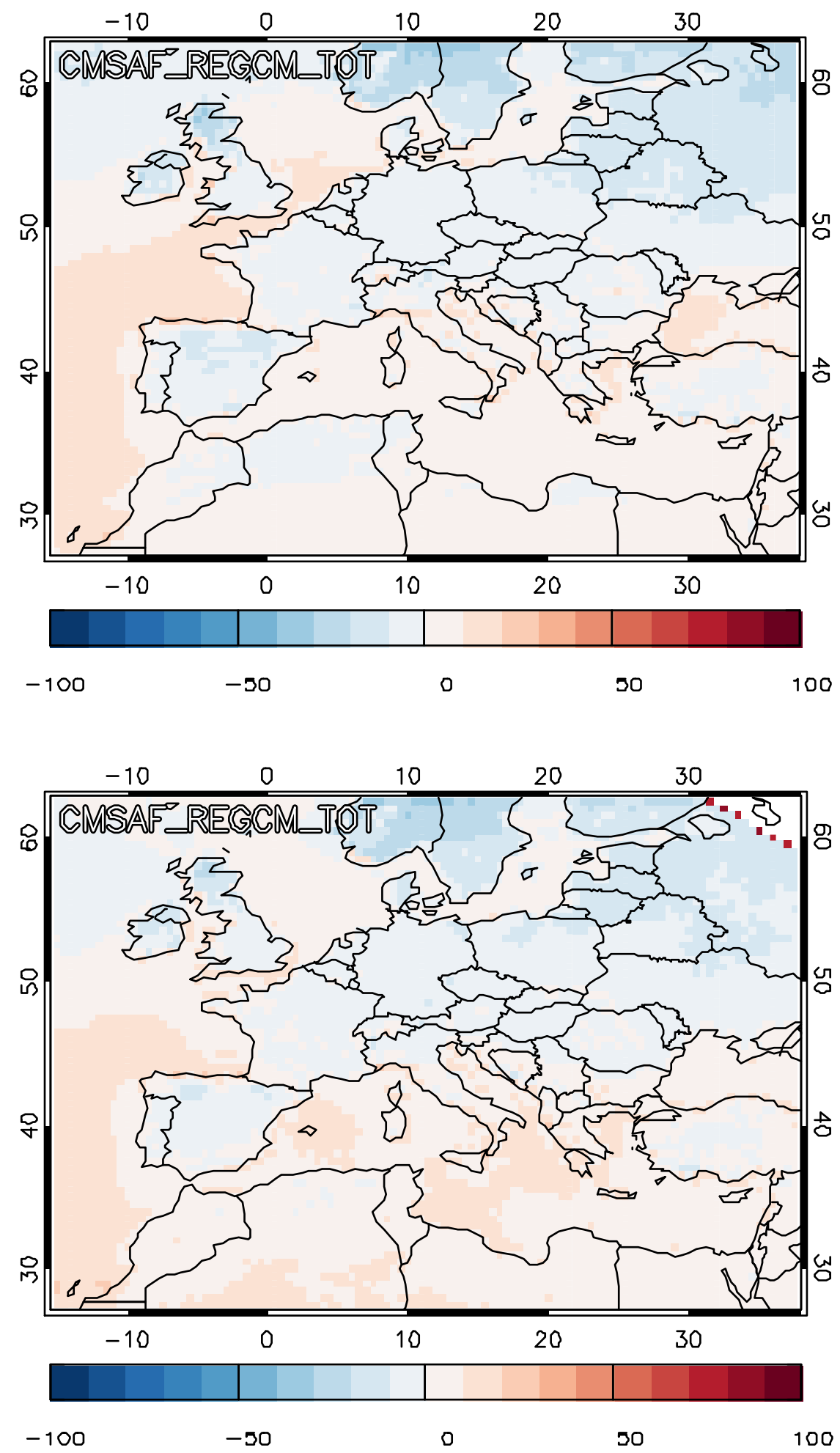

Figure S2. Normalized mean bias (NMB) patterns of RegCM4-CM SAF SSR over Europe from (a) MFG MVIRI (2000-2005) and (b) MSG SEVIRI (2006-2009) observations. 
a)

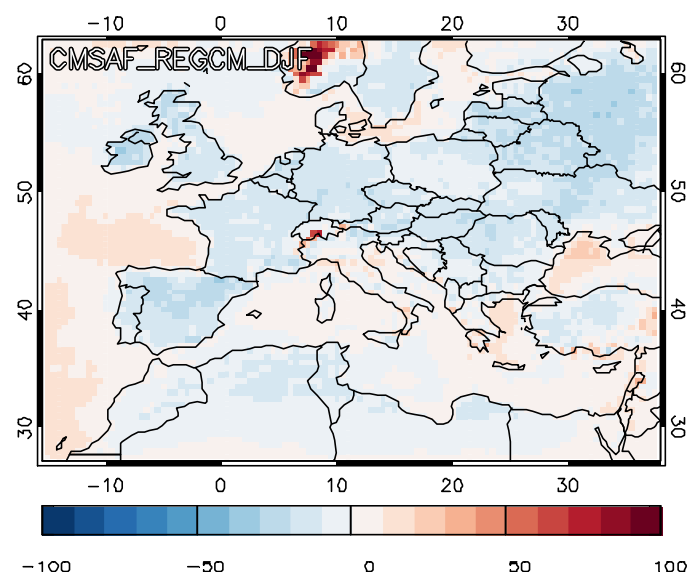

c)

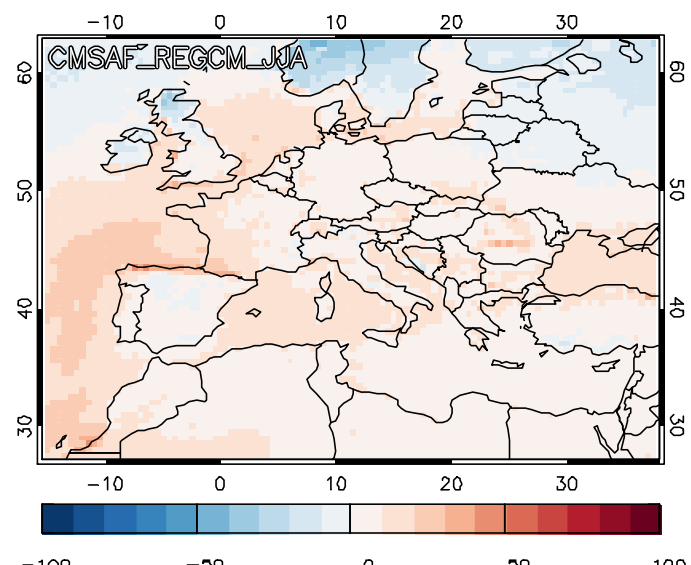

b)

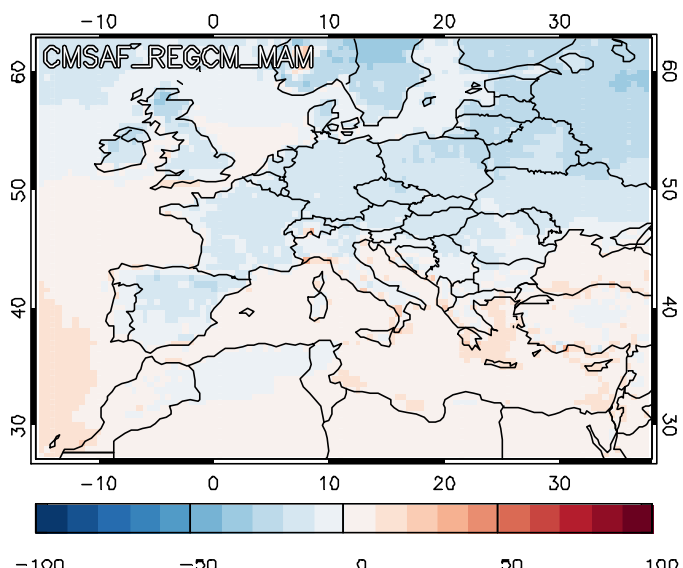

d)

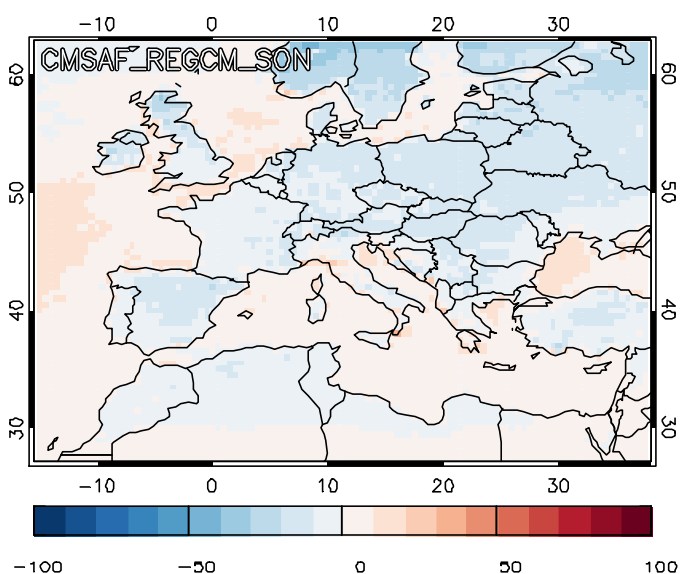

Figure S3. Seasonal Normalized mean bias (NMB) patterns of RegCM4-CM SAF SSR over Europe for (a) winter (DJF), (b) spring (MAM), (c) summer (JJA) and (d) autumn (SON) from MFG MVIRI observations. 
a)

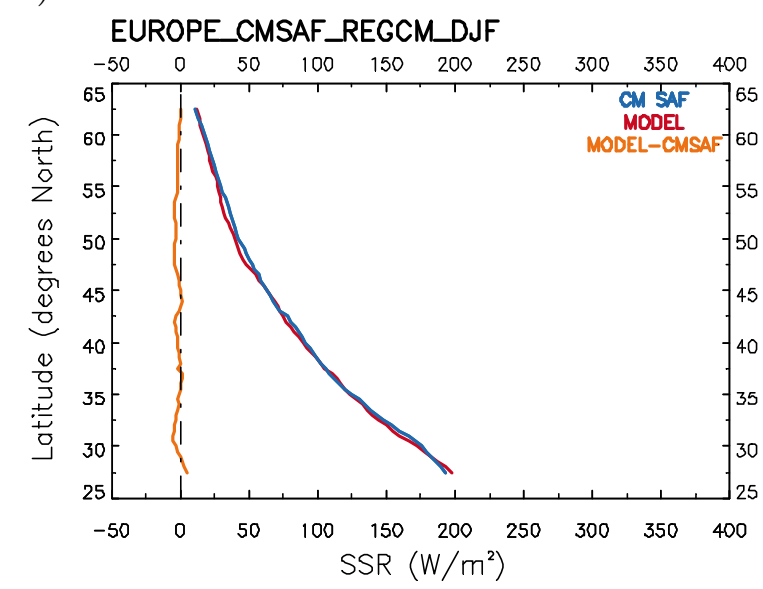

c)

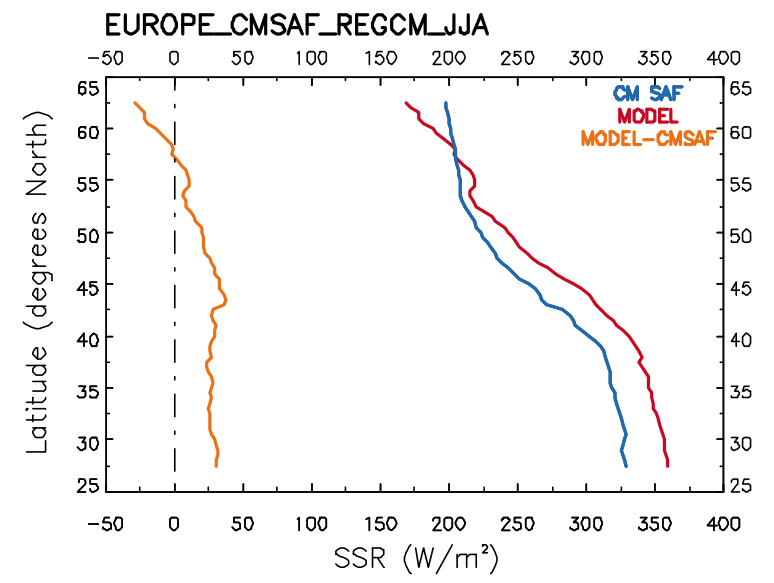

b)

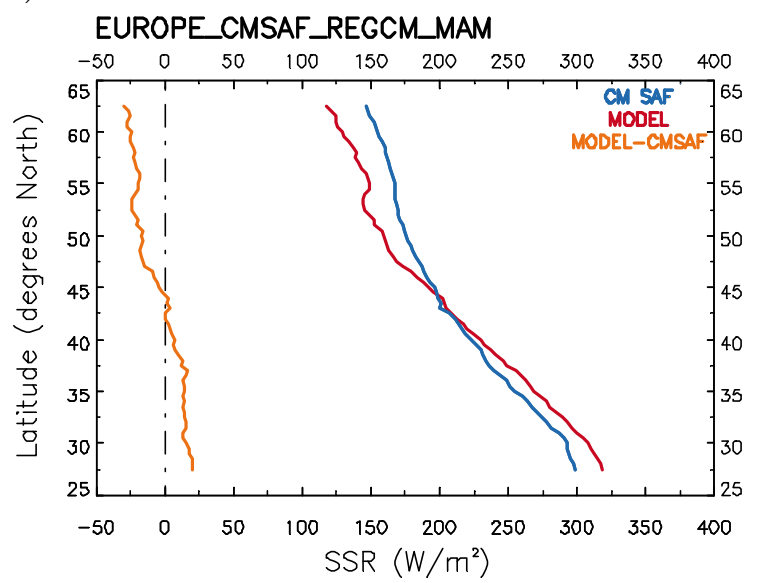

d)

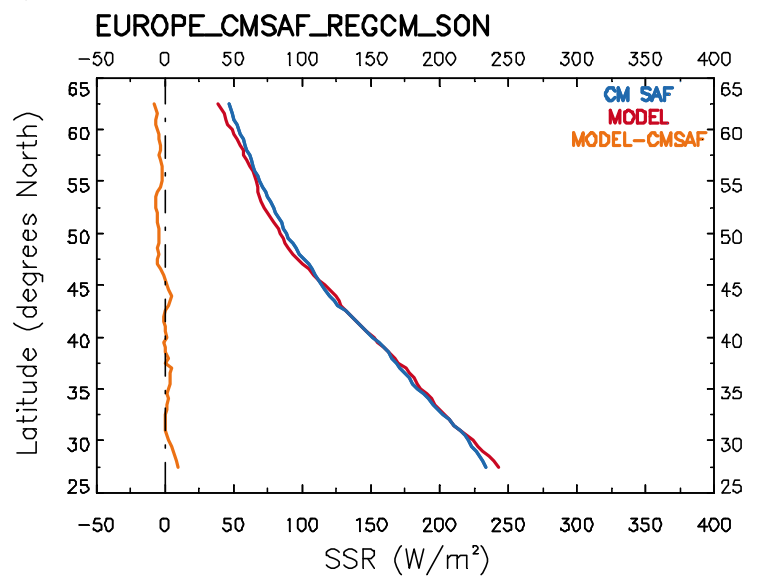

Figure S4. Latitudinal variability of RegCM4 SSR (red), CM SAF SSR (blue) and their difference (orange) over Europe for (a) winter (DJF), (b) spring (MAM), (c) summer (JJA) and (d) autumn (SON) from MFG MVIRI observations. 
a)

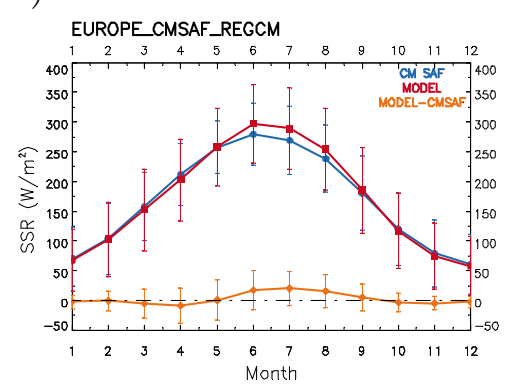

d)

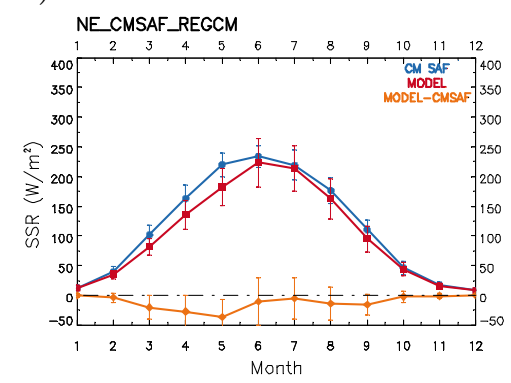

g)

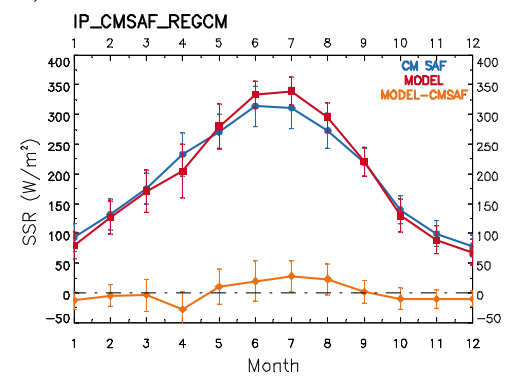

j)

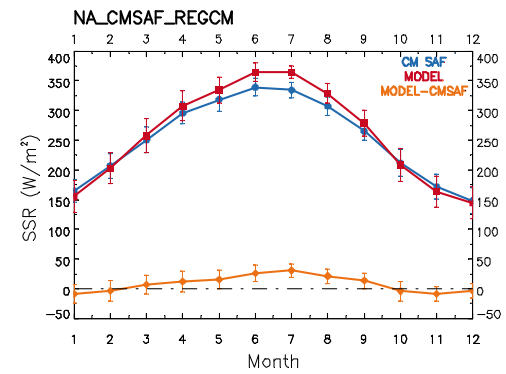

b)

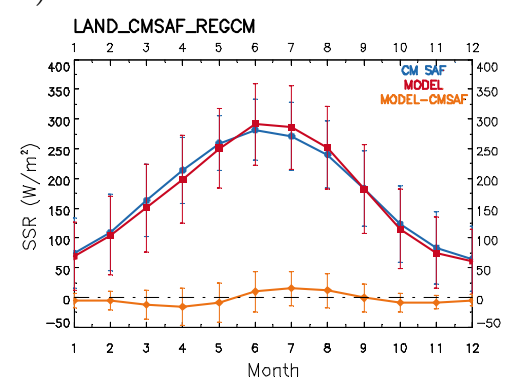

e)

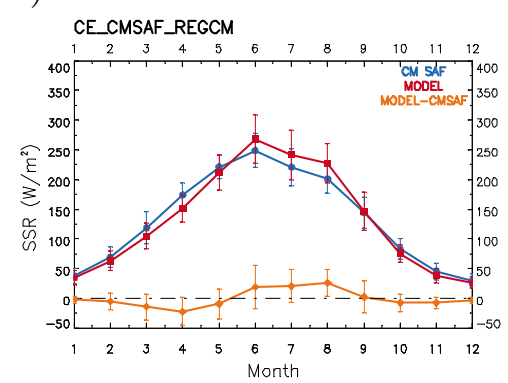

h)

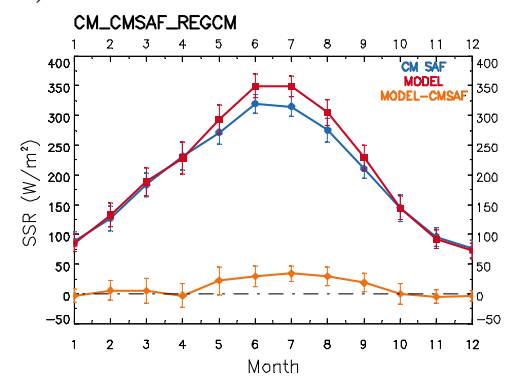

c)

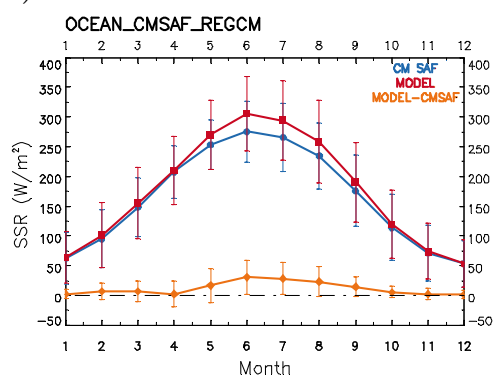

f)

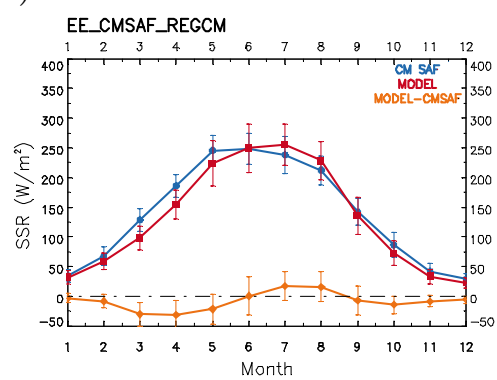

i)

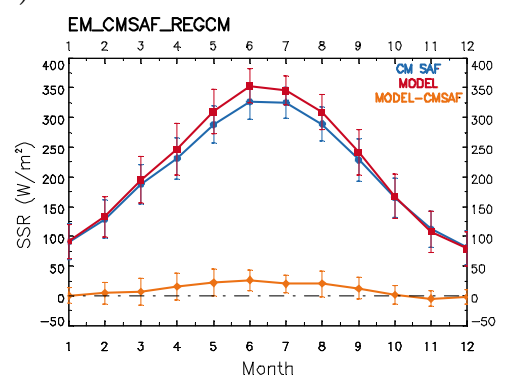

Figure S5. Seasonal variability of RegCM4 SSR (red), CM SAF SSR (blue) and their difference (orange) over (a) the whole Europe, (b) Land, (c) Ocean, (d) NE, (e) CE, (f) EE, (g) IP, (h) CM, (i) EM, (j) NA from MFG MVIRI observations. 
Table S1. Average RegCM4 SSR, CM SAF SSR (MFG MVIRI), their difference, the corresponding Normalized Mean Bias (NMB) and the statistical significance of the results at the $95 \%$ confidence level due to a two independent sample t-test per season ${ }^{1}$ and region. The NSD, MNMB and RMSE are also given along with Pearson's correlation coefficient $(\mathrm{R})$, the number of observations used and the coefficients a and $\mathrm{b}$ of the regression line $(\mathrm{RegCM} 4=\mathrm{a} \cdot \mathrm{CMSAF}+\mathrm{b})$. The region are listed in alphabetic order.

\begin{tabular}{|c|c|c|c|c|c|c|c|c|c|c|c|c|c|}
\hline Season & Region & REG & CMS & Diff. & NMB & Signif. & $\mathrm{R}$ & Obs. & $\mathrm{b}$ & $\mathrm{a}$ & NSD & MNMB & RMSE \\
\hline DJF & $\mathrm{CE}$ & 41.57 & 45.48 & -3.91 & -8.60 & 1 & 0.90 & 13986 & 3.48 & 0.84 & 0.93 & -8.70 & 10.53 \\
\hline JJA & $\mathrm{CE}$ & 245.32 & 223.63 & 21.69 & 9.70 & 1 & 0.72 & 13986 & 47.85 & 0.88 & 1.23 & 8.89 & 36.65 \\
\hline MAM & $\mathrm{CE}$ & 155.88 & 171.25 & -15.37 & -8.97 & 1 & 0.88 & 13986 & -4.02 & 0.93 & 1.06 & -10.43 & 28.55 \\
\hline SON & $\mathrm{CE}$ & 86.45 & 90.76 & -4.31 & -4.75 & 1 & 0.93 & 13986 & -6.13 & 1.02 & 1.10 & -8.82 & 19.43 \\
\hline TOT & $\mathrm{CE}$ & 132.31 & 132.78 & -0.48 & -0.36 & 0 & 0.96 & 55944 & -8.82 & 1.06 & 1.11 & -4.76 & 25.72 \\
\hline DJF & $\mathrm{CM}$ & 96.69 & 96.75 & -0.06 & -0.06 & 0 & 0.90 & 8262 & 0.57 & 0.99 & 1.10 & -0.90 & 13.38 \\
\hline JJA & $\mathrm{CM}$ & 334.50 & 303.32 & 31.18 & 10.28 & 1 & 0.84 & 8262 & 60.38 & 0.90 & 1.07 & 9.79 & 34.82 \\
\hline MAM & $\mathrm{CM}$ & 236.73 & 228.59 & 8.14 & 3.56 & 1 & 0.88 & 8262 & -3.93 & 1.05 & 1.20 & 2.95 & 25.17 \\
\hline SON & $\mathrm{CM}$ & 154.90 & 150.33 & 4.57 & 3.04 & 1 & 0.96 & 8262 & -15.63 & 1.13 & 1.18 & 1.15 & 18.51 \\
\hline TOT & $\mathrm{CM}$ & 205.70 & 194.75 & 10.96 & 5.63 & 1 & 0.98 & 33048 & -13.65 & 1.13 & 1.15 & 3.25 & 24.33 \\
\hline DJF & $\mathrm{EE}$ & 37.97 & 43.54 & -5.57 & -12.80 & 1 & 0.90 & 15498 & 1.95 & 0.83 & 0.91 & -15.38 & 10.51 \\
\hline JJA & $\mathrm{EE}$ & 244.18 & 233.06 & 11.12 & 4.77 & 1 & 0.68 & 15498 & 50.57 & 0.83 & 1.22 & 4.37 & 30.33 \\
\hline MAM & $\mathrm{EE}$ & 158.72 & 186.67 & -27.95 & -14.97 & 1 & 0.91 & 15498 & -32.53 & 1.02 & 1.12 & -18.94 & 36.93 \\
\hline SON & $\mathrm{EE}$ & 80.46 & 90.20 & -9.74 & -10.80 & 1 & 0.93 & 15498 & -7.34 & 0.97 & 1.05 & -15.74 & 20.03 \\
\hline TOT & $\mathrm{EE}$ & 130.33 & 138.37 & -8.03 & -5.81 & 1 & 0.96 & 61992 & -10.31 & 1.02 & 1.06 & -11.42 & 26.43 \\
\hline DJF & $\mathrm{EM}$ & 101.43 & 100.43 & 1.00 & 0.99 & 1 & 0.92 & 19926 & 4.25 & 0.97 & 1.05 & 0.64 & 14.84 \\
\hline JJA & $\mathrm{EM}$ & 335.73 & 313.39 & 22.34 & 7.13 & 1 & 0.85 & 19926 & 64.10 & 0.87 & 1.02 & 6.92 & 28.96 \\
\hline MAM & $\mathrm{EM}$ & 250.43 & 235.66 & 14.78 & 6.27 & 1 & 0.93 & 19926 & -5.74 & 1.09 & 1.17 & 5.43 & 27.72 \\
\hline SON & $\mathrm{EM}$ & 171.91 & 168.63 & 3.28 & 1.95 & 1 & 0.97 & 19926 & -12.50 & 1.09 & 1.13 & 0.05 & 17.78 \\
\hline TOT & $\mathrm{EM}$ & 214.87 & 204.53 & 10.35 & 5.06 & 1 & 0.98 & 79704 & -8.19 & 1.09 & 1.11 & 3.26 & 23.15 \\
\hline DJF & $\mathrm{EU}$ & 76.23 & 78.11 & -1.87 & -2.40 & 1 & 0.98 & 135468 & -0.97 & 0.99 & 1.01 & -3.69 & 12.64 \\
\hline JJA & $\mathrm{EU}$ & 280.00 & 262.24 & 17.76 & 6.77 & 1 & 0.91 & 135468 & -12.28 & 1.11 & 1.23 & 5.42 & 35.08 \\
\hline MAM & $\mathrm{EU}$ & 204.26 & 208.78 & -4.53 & -2.17 & 1 & 0.94 & 135468 & -33.21 & 1.14 & 1.22 & -5.20 & 30.05 \\
\hline SON & $\mathrm{EU}$ & 125.37 & 126.35 & -0.98 & -0.78 & 1 & 0.98 & 135468 & -7.76 & 1.05 & 1.08 & -4.17 & 17.33 \\
\hline TOT & $\mathrm{EU}$ & 171.47 & 168.87 & 2.59 & 1.54 & 1 & 0.97 & 541872 & -10.81 & 1.08 & 1.11 & -1.91 & 25.46 \\
\hline DJF & IP & 91.95 & 101.17 & -9.22 & -9.11 & 1 & 0.89 & 6426 & -5.72 & 0.97 & 1.09 & -11.54 & 18.46 \\
\hline JJA & IP & 322.84 & 299.11 & 23.73 & 7.94 & 1 & 0.65 & 6426 & 163.21 & 0.53 & 0.82 & 7.99 & 37.40 \\
\hline MAM & IP & 218.76 & 225.96 & -7.21 & -3.19 & 1 & 0.84 & 6426 & -9.72 & 1.01 & 1.20 & -4.45 & 33.36 \\
\hline SON & IP & 146.42 & 152.64 & -6.21 & -4.07 & 1 & 0.95 & 6426 & -13.92 & 1.05 & 1.10 & -6.36 & 19.59 \\
\hline TOT & IP & 194.99 & 194.72 & 0.27 & 0.14 & 0 & 0.96 & 25704 & -18.12 & 1.09 & 1.14 & -3.59 & 28.45 \\
\hline DJF & LA & 77.83 & 82.68 & -4.85 & -5.86 & 1 & 0.98 & 85518 & -2.91 & 0.98 & 1.00 & -8.59 & 14.17 \\
\hline JJA & LA & 276.53 & 264.43 & 12.10 & 4.58 & 1 & 0.91 & 85518 & -21.39 & 1.13 & 1.24 & 3.18 & 32.68 \\
\hline MAM & LA & 199.88 & 212.10 & -12.22 & -5.76 & 1 & 0.94 & 85518 & -44.55 & 1.15 & 1.23 & -9.66 & 32.35 \\
\hline SON & LA & 123.93 & 129.85 & -5.92 & -4.56 & 1 & 0.98 & 85518 & -12.76 & 1.05 & 1.08 & -9.85 & 18.42 \\
\hline TOT & LA & 169.55 & 172.30 & -2.75 & -1.59 & 1 & 0.97 & 342072 & -14.98 & 1.07 & 1.10 & -6.26 & 25.67 \\
\hline DJF & NA & 167.82 & 173.18 & -5.37 & -3.10 & 1 & 0.91 & 19440 & -14.23 & 1.05 & 1.15 & -3.88 & 16.08 \\
\hline JJA & $\mathrm{NA}$ & 352.74 & 326.82 & 25.92 & 7.93 & 1 & 0.82 & 19440 & 53.24 & 0.92 & 1.12 & 7.61 & 28.98 \\
\hline MAM & NA & 299.97 & 288.23 & 11.74 & 4.07 & 1 & 0.91 & 19440 & -5.50 & 1.06 & 1.17 & 3.77 & 20.59 \\
\hline SON & NA & 216.44 & 216.16 & 0.28 & 0.13 & 0 & 0.96 & 19440 & -45.33 & 1.21 & 1.26 & -1.09 & 17.13 \\
\hline TOT & NA & 259.24 & 251.10 & 8.14 & 3.24 & 1 & 0.98 & 77760 & -35.56 & 1.17 & 1.20 & 1.61 & 21.31 \\
\hline DJF & $\mathrm{NE}$ & 18.63 & 20.14 & -1.52 & -7.53 & 1 & 0.94 & 16614 & 2.12 & 0.82 & 0.87 & -3.67 & 5.40 \\
\hline JJA & $\mathrm{NE}$ & 199.86 & 209.66 & -9.80 & -4.67 & 1 & 0.67 & 16614 & -0.78 & 0.96 & 1.43 & -6.33 & 35.83 \\
\hline MAM & $\mathrm{NE}$ & 133.38 & 161.50 & -28.13 & -17.42 & 1 & 0.86 & 16614 & 5.84 & 0.79 & 0.92 & -19.97 & 39.08 \\
\hline SON & $\mathrm{NE}$ & 51.53 & 57.89 & -6.36 & -10.98 & 1 & 0.95 & 16614 & 3.51 & 0.83 & 0.87 & -9.81 & 14.73 \\
\hline TOT & $\mathrm{NE}$ & 100.85 & 112.30 & -11.45 & -10.20 & 1 & 0.96 & 66456 & -0.65 & 0.90 & 0.95 & -9.95 & 27.64 \\
\hline DJF & $\mathrm{OC}$ & 73.45 & 70.07 & 3.38 & 4.83 & 1 & 0.98 & 49950 & 0.14 & 1.05 & 1.07 & 4.88 & 11.00 \\
\hline JJA & $\mathrm{OC}$ & 285.95 & 258.49 & 27.46 & 10.62 & 1 & 0.92 & 49950 & -1.45 & 1.11 & 1.20 & 9.24 & 38.84 \\
\hline MAM & $\mathrm{OC}$ & 211.75 & 203.10 & 8.65 & 4.26 & 1 & 0.95 & 49950 & -19.73 & 1.14 & 1.19 & 2.45 & 25.63 \\
\hline SON & $\mathrm{OC}$ & 127.83 & 120.36 & 7.47 & 6.21 & 1 & 0.99 & 49950 & -1.32 & 1.07 & 1.09 & 5.56 & 15.28 \\
\hline TOT & $\mathrm{OC}$ & 174.74 & 163.00 & 11.74 & 7.20 & 1 & 0.98 & 199800 & -5.31 & 1.10 & 1.12 & 5.53 & 25.10 \\
\hline
\end{tabular}

${ }^{1}$ TOT stands for the whole dataset, DJF for December-January-February, MAM for March-April-May, JJA for June-July-August and SON for September-October-November. Regions are listed in alphabetical order. 
Table S2. Average RegCM4 SSR, CM SAF SSR (MSG SEVIRI), their difference, the corresponding NMB and the statistical significance of the results at the $95 \%$ confidence level due to a two independent sample t-test per season and region. The NSD, MNMB and RMSE are also given along with Pearson's correlation coefficient $(\mathrm{R})$, the number of observations used and the coefficients $a$ and $b$ of the regression line $(\mathrm{RegCM} 4=\mathrm{a} \cdot \mathrm{CMSAF}+\mathrm{b})$. The region are listed in alphabetic order.

\begin{tabular}{|c|c|c|c|c|c|c|c|c|c|c|c|c|c|}
\hline Season & Region & REG & CMS & Diff. & NMB & Signif. & $\mathrm{R}$ & Obs. & $\mathrm{b}$ & $\mathrm{a}$ & NSD & MNMB & RMSE \\
\hline DJF & $\mathrm{CE}$ & 42.30 & 42.75 & -0.45 & -1.05 & 0 & 0.86 & 8547 & 11.08 & 0.73 & 0.85 & 11.11 & 12.57 \\
\hline JJA & $\mathrm{CE}$ & 245.57 & 228.91 & 16.66 & 7.28 & 1 & 0.79 & 9324 & 17.85 & 0.99 & 1.25 & 6.42 & 33.59 \\
\hline MAM & $\mathrm{CE}$ & 158.12 & 174.03 & -15.91 & -9.14 & 1 & 0.84 & 9324 & -0.14 & 0.91 & 1.08 & -11.09 & 34.51 \\
\hline SON & $\mathrm{CE}$ & 84.38 & 90.92 & -6.54 & -7.19 & 1 & 0.94 & 9324 & 1.15 & 0.92 & 0.97 & -8.15 & 17.49 \\
\hline TOT & $\mathrm{CE}$ & 134.52 & 136.10 & -1.58 & -1.16 & 1 & 0.96 & 36519 & -5.04 & 1.03 & 1.07 & -0.67 & 26.59 \\
\hline DJF & $\mathrm{CM}$ & 97.29 & 96.68 & 0.61 & 0.63 & 0 & 0.92 & 5049 & 2.30 & 0.98 & 1.07 & 0.28 & 11.62 \\
\hline JJA & $\mathrm{CM}$ & 331.26 & 299.93 & 31.34 & 10.45 & 1 & 0.82 & 5508 & 62.57 & 0.90 & 1.09 & 9.93 & 35.08 \\
\hline MAM & $\mathrm{CM}$ & 243.68 & 225.86 & 17.82 & 7.89 & 1 & 0.91 & 5508 & -20.14 & 1.17 & 1.28 & 6.53 & 31.07 \\
\hline $\mathrm{SON}$ & $\mathrm{CM}$ & 157.71 & 149.80 & 7.90 & 5.28 & 1 & 0.95 & 5508 & -9.18 & 1.11 & 1.18 & 3.68 & 19.89 \\
\hline TOT & $\mathrm{CM}$ & 209.83 & 195.12 & 14.71 & 7.54 & 1 & 0.98 & 21573 & -12.36 & 1.14 & 1.16 & 5.21 & 26.33 \\
\hline DJF & $\mathrm{EE}$ & 37.53 & 38.84 & -1.31 & -3.38 & 1 & 0.90 & 9471 & 10.00 & 0.71 & 0.79 & 10.90 & 10.14 \\
\hline JJA & $\mathrm{EE}$ & 248.43 & 242.79 & 5.63 & 2.32 & 1 & 0.79 & 10332 & 11.72 & 0.97 & 1.23 & 1.75 & 27.91 \\
\hline MAM & $\mathrm{EE}$ & 155.22 & 179.35 & -24.13 & -13.45 & 1 & 0.89 & 10332 & -13.70 & 0.94 & 1.06 & -16.36 & 37.11 \\
\hline $\mathrm{SON}$ & $\mathrm{EE}$ & 80.07 & 88.77 & -8.70 & -9.80 & 1 & 0.96 & 10332 & -0.59 & 0.91 & 0.94 & -11.31 & 15.62 \\
\hline TOT & $\mathrm{EE}$ & 132.29 & 139.54 & -7.25 & -5.20 & 1 & 0.96 & 40467 & -5.52 & 0.99 & 1.02 & -4.07 & 25.24 \\
\hline DJF & EM & 105.08 & 101.76 & 3.31 & 3.25 & 1 & 0.92 & 12177 & 2.68 & 1.01 & 1.09 & 2.75 & 14.67 \\
\hline JJA & EM & 339.32 & 312.76 & 26.56 & 8.49 & 1 & 0.84 & 13284 & 68.89 & 0.86 & 1.03 & 8.18 & 31.21 \\
\hline MAM & EM & 251.44 & 235.60 & 15.85 & 6.73 & 1 & 0.94 & 13284 & -27.95 & 1.19 & 1.26 & 5.05 & 30.27 \\
\hline SON & EM & 171.81 & 163.66 & 8.16 & 4.98 & 1 & 0.96 & 13284 & -5.70 & 1.08 & 1.13 & 3.38 & 19.57 \\
\hline TOT & $\mathrm{EM}$ & 219.29 & 205.61 & 13.68 & 6.66 & 1 & 0.98 & 52029 & -8.06 & 1.11 & 1.13 & 4.89 & 25.12 \\
\hline DJF & $\mathrm{EU}$ & 77.08 & 74.17 & 2.91 & 3.93 & 1 & 0.98 & 82238 & 4.79 & 0.97 & 1.00 & 29.58 & 12.54 \\
\hline JJA & $\mathrm{EU}$ & 281.62 & 265.18 & 16.44 & 6.20 & 1 & 0.90 & 89760 & -25.13 & 1.16 & 1.28 & 4.73 & 35.38 \\
\hline MAM & $\mathrm{EU}$ & 206.76 & 206.67 & 0.09 & 0.04 & 0 & 0.93 & 89760 & -32.42 & 1.16 & 1.24 & -3.02 & 31.42 \\
\hline SON & $\mathrm{EU}$ & 126.27 & 123.31 & 2.96 & 2.40 & 1 & 0.97 & 89736 & -4.17 & 1.06 & 1.09 & 5.74 & 18.21 \\
\hline TOT & $\mathrm{EU}$ & 174.99 & 169.33 & 5.66 & 3.34 & 1 & 0.97 & 351494 & -6.32 & 1.07 & 1.10 & 8.82 & 26.33 \\
\hline DJF & IP & 91.71 & 98.58 & -6.87 & -6.97 & 1 & 0.87 & 3927 & 7.46 & 0.85 & 0.98 & -7.44 & 15.42 \\
\hline JJA & IP & 317.51 & 296.28 & 21.23 & 7.17 & 1 & 0.68 & 4284 & 136.21 & 0.61 & 0.90 & 7.11 & 32.57 \\
\hline MAM & IP & 224.84 & 223.98 & 0.87 & 0.39 & 0 & 0.87 & 4284 & -13.79 & 1.07 & 1.22 & -0.91 & 27.62 \\
\hline SON & IP & 148.61 & 151.77 & -3.17 & -2.09 & 1 & 0.94 & 4284 & -4.02 & 1.01 & 1.07 & -3.40 & 18.65 \\
\hline TOT & IP & 197.88 & 194.66 & 3.22 & 1.66 & 1 & 0.97 & 16779 & -14.73 & 1.09 & 1.13 & -1.03 & 24.70 \\
\hline DJF & LA & 78.12 & 78.01 & 0.11 & 0.14 & 0 & 0.98 & 51735 & 1.66 & 0.98 & 1.00 & 21.91 & 12.76 \\
\hline JJA & LA & 278.63 & 266.99 & 11.64 & 4.36 & 1 & 0.90 & 56484 & -34.83 & 1.17 & 1.30 & 2.77 & 34.69 \\
\hline MAM & LA & 202.65 & 208.71 & -6.06 & -2.90 & 1 & 0.93 & 56484 & -40.87 & 1.17 & 1.25 & -6.72 & 33.35 \\
\hline $\mathrm{SON}$ & LA & 124.87 & 126.07 & -1.20 & -0.95 & 1 & 0.98 & 56460 & -8.61 & 1.06 & 1.09 & 0.20 & 18.05 \\
\hline TOT & LA & 173.07 & 171.92 & 1.15 & 0.67 & 1 & 0.97 & 221163 & -10.40 & 1.07 & 1.10 & 4.17 & 26.70 \\
\hline DJF & NA & 164.74 & 161.80 & 2.94 & 1.81 & 1 & 0.93 & 11880 & -1.61 & 1.03 & 1.10 & 1.44 & 13.19 \\
\hline JJA & NA & 353.48 & 320.52 & 32.96 & 10.28 & 1 & 0.77 & 12960 & 118.57 & 0.73 & 0.95 & 9.83 & 35.89 \\
\hline MAM & NA & 303.81 & 280.20 & 23.61 & 8.43 & 1 & 0.90 & 12960 & -5.06 & 1.10 & 1.22 & 7.83 & 29.87 \\
\hline $\mathrm{SON}$ & NA & 217.20 & 205.79 & 11.41 & 5.54 & 1 & 0.95 & 12960 & -25.80 & 1.18 & 1.25 & 4.52 & 20.89 \\
\hline TOT & NA & 261.83 & 243.79 & 18.04 & 7.40 & 1 & 0.98 & 50760 & -21.07 & 1.16 & 1.18 & 6.00 & 26.63 \\
\hline DJF & $\mathrm{NE}$ & 19.31 & 12.67 & 6.64 & 52.45 & 1 & 0.91 & 9810 & 11.08 & 0.65 & 0.72 & 118.94 & 10.19 \\
\hline JJA & $\mathrm{NE}$ & 198.68 & 219.39 & -20.71 & -9.44 & 1 & 0.72 & 10740 & 32.98 & 0.76 & 1.05 & -10.51 & 39.29 \\
\hline MAM & $\mathrm{NE}$ & 137.62 & 160.43 & -22.81 & -14.22 & 1 & 0.91 & 10740 & 8.89 & 0.80 & 0.88 & -15.28 & 33.68 \\
\hline SON & $\mathrm{NE}$ & 52.88 & 53.42 & -0.53 & -1.00 & 0 & 0.94 & 10720 & 9.69 & 0.81 & 0.86 & 26.59 & 15.74 \\
\hline TOT & $\mathrm{NE}$ & 103.98 & 113.69 & -9.71 & -8.54 & 1 & 0.97 & 42010 & 8.60 & 0.84 & 0.87 & 27.97 & 27.79 \\
\hline DJF & $\mathrm{OC}$ & 75.32 & 67.66 & 7.66 & 11.32 & 1 & 0.98 & 30503 & 9.00 & 0.98 & 1.00 & 42.58 & 12.16 \\
\hline JJA & $\mathrm{OC}$ & 286.69 & 262.11 & 24.57 & 9.37 & 1 & 0.93 & 33276 & -12.49 & 1.14 & 1.23 & 8.05 & 36.52 \\
\hline MAM & $\mathrm{OC}$ & 213.75 & 203.22 & 10.53 & 5.18 & 1 & 0.95 & 33276 & -20.68 & 1.15 & 1.21 & 3.26 & 27.83 \\
\hline SON & $\mathrm{OC}$ & 128.65 & 118.63 & 10.02 & 8.45 & 1 & 0.98 & 33276 & 1.98 & 1.07 & 1.09 & 15.13 & 18.49 \\
\hline TOT & $\mathrm{OC}$ & 178.24 & 164.93 & 13.31 & 8.07 & 1 & 0.98 & 130331 & -0.40 & 1.08 & 1.10 & 16.72 & 25.69 \\
\hline
\end{tabular}




\section{Cloud Fractional Cover}

Table S3. Average RegCM4 SSR and CM SAF CFC (MSG SEVIRI) with their standard deviations $( \pm 1 \sigma)$ and the corresponding NMB per season and region. When the difference between RegCM4 and CM SAF CFC is statistically significant at the $95 \%$ confidence level due to a two independent sample t-test, the NMB values are marked with bold letters while in the opposite case they are marked with an asterisk. Positive NMBs are marked with red color while negative NMBs with blue. ANN corresponds to annual, DJF to winter, MAM to spring, JJA to summer and SON to autumn results while the regions are listed in alphabetical order.

\begin{tabular}{|c|c|c|c|c|c|c|c|c|c|c|c|c|c|c|c|}
\hline & \multicolumn{3}{|c|}{$\mathrm{ANN}$} & \multicolumn{3}{|c|}{ DJF } & \multicolumn{3}{|c|}{ MAM } & \multicolumn{3}{|c|}{ JJA } & \multicolumn{3}{|c|}{ SON } \\
\hline & MOD & SAT & bias & MOD & SAT & bias & MOD & SAT & bias & MOD & SAT & bias & MOD & SAT & bias \\
\hline $\mathrm{CE}$ & $0.55 \pm 0.11$ & $0.69 \pm 0.12$ & -19.73 & $0.62 \pm 0.06$ & $0.74 \pm 0.12$ & -16.00 & $0.58 \pm 0.07$ & $0.67 \pm 0.112$ & -13.95 & $0.42 \pm 0.11$ & $0.65 \pm 0.123$ & -34.80 & $0.58 \pm 0.08$ & $0.68 \pm 0.13$ & -14.96 \\
\hline $\mathrm{CM}$ & $0.35 \pm 0.16$ & $0.51 \pm 0.17$ & -31.19 & $0.47 \pm 0.08$ & $0.64 \pm 0.112$ & -25.92 & $0.41 \pm 0.1$ & $0.57 \pm 0.105$ & -28.05 & $0.14 \pm 0.11$ & $0.30 \pm 0.125$ & -54.86 & $0.39 \pm 0.11$ & $0.54 \pm 0.12$ & -27.26 \\
\hline $\mathrm{EE}$ & $0.56 \pm 0.14$ & $0.67 \pm 0.15$ & -15.99 & $0.67 \pm 0.05$ & $0.8 \pm 0.085$ & -16.84 & $0.59 \pm 0.09$ & $0.65 \pm 0.117$ & -9.20 & $0.4 \pm 0.12$ & $0.55 \pm 0.129$ & -27.46 & $0.58 \pm 0.1$ & $0.67 \pm 0.14$ & -12.21 \\
\hline EM & $0.32 \pm 0.2$ & $0.44 \pm 0.24$ & -28.83 & $0.49 \pm 0.13$ & $0.66 \pm 0.147$ & -25.67 & $0.36 \pm 0.16$ & $0.51 \pm 0.156$ & -28.60 & $0.1 \pm 0.11$ & $0.18 \pm 0.147$ & -45.93 & $0.33 \pm 0.17$ & $0.44 \pm 0.20$ & -26.57 \\
\hline $\mathrm{EU}$ & $0.44 \pm 0.2$ & $0.58 \pm 0.25$ & -24.34 & $0.53 \pm 0.15$ & $0.68 \pm 0.229$ & -21.81 & $0.47 \pm 0.17$ & $0.59 \pm 0.209$ & -20.53 & $0.3 \pm 0.21$ & $0.47 \pm 0.281$ & -36.57 & $0.47 \pm 0.18$ & $0.59 \pm 0.24$ & -21.14 \\
\hline IP & $0.41 \pm 0.16$ & $0.48 \pm 0.17$ & -13.65 & $0.5 \pm 0.10$ & $0.54 \pm 0.143$ & -7.68 & $0.48 \pm 0.11$ & $0.56 \pm 0.134$ & -14.37 & $0.23 \pm 0.13$ & $0.33 \pm 0.169$ & -30.11 & $0.45 \pm 0.12$ & $0.49 \pm 0.15$ & -7.95 \\
\hline LA & $0.45 \pm 0.2$ & $0.53 \pm 0.24$ & -13.74 & $0.54 \pm 0.17$ & $0.61 \pm 0.24$ & -11.53 & $0.48 \pm 0.17$ & $0.54 \pm 0.205$ & -9.82 & $0.31 \pm 0.20$ & $0.43 \pm 0.257$ & -26.92 & $0.48 \pm 0.18$ & $0.53 \pm 0.24$ & -9.44 \\
\hline NA & $0.23 \pm 0.14$ & $0.23 \pm 0.14$ & $0.36^{*}$ & $0.3 \pm 0.11$ & $0.27 \pm 0.137$ & 8.12 & $0.26 \pm 0.11$ & $0.26 \pm 0.122$ & -2.27 & $0.11 \pm 0.10$ & $0.15 \pm 0.128$ & -29.22 & $0.28 \pm 0.14$ & $0.25 \pm 0.14$ & 13.14 \\
\hline $\mathrm{NE}$ & $0.59 \pm 0.09$ & $0.75 \pm 0.12$ & -20.34 & $0.65 \pm 0.05$ & $0.83 \pm 0.092$ & -21.20 & $0.59 \pm 0.07$ & $0.68 \pm 0.106$ & -12.79 & $0.52 \pm 0.09$ & $0.7 \pm 0.107$ & -25.63 & $0.62 \pm 0.07$ & $0.78 \pm 0.11$ & -21.39 \\
\hline $\mathrm{OC}$ & $0.42 \pm 0.19$ & $0.68 \pm 0.23$ & -38.41 & $0.52 \pm 0.12$ & $0.8 \pm 0.142$ & -35.23 & $0.44 \pm 0.15$ & $0.68 \pm 0.184$ & -34.98 & $0.27 \pm 0.21$ & $0.54 \pm 0.306$ & -49.63 & $0.44 \pm 0.17$ & $0.7 \pm 0.27$ & -36.50 \\
\hline
\end{tabular}


Table S4. Average RegCM4 CFC, CM SAF CFC (MSG SEVIRI), their difference, the corresponding NMB and the statistical significance of the results at the $95 \%$ confidence level due to a two independent sample t-test per season and region. The NSD, MNMB and the RMSE are also given along with Pearson's correlation coefficient (R), the number of observations used and the coefficients $\mathrm{a}$ and $\mathrm{b}$ of the regression line $(\mathrm{RegCM} 4=\mathrm{a} \cdot \mathrm{CMSAF}+\mathrm{b})$. The region are listed in alphabetic order.

\begin{tabular}{|c|c|c|c|c|c|c|c|c|c|c|c|c|c|}
\hline Season & Region & REG & CMS & Diff. & NMB & Signif. & $\mathrm{R}$ & Obs. & $\mathrm{b}$ & $\mathrm{a}$ & NSD & MNMB & RMSE \\
\hline DJF & $\mathrm{CE}$ & 0.62 & 0.74 & -0.12 & -16.01 & 1 & 0.13 & 13209 & 0.57 & 0.07 & 0.52 & -16.33 & 0.17 \\
\hline JJA & $\mathrm{CE}$ & 0.42 & 0.65 & -0.23 & -34.80 & 1 & 0.53 & 13986 & 0.11 & 0.48 & 0.90 & -43.29 & 0.25 \\
\hline MAM & $\mathrm{CE}$ & 0.58 & 0.67 & -0.09 & -13.96 & 1 & 0.23 & 13986 & 0.48 & 0.15 & 0.66 & -14.14 & 0.15 \\
\hline SON & $\mathrm{CE}$ & 0.58 & 0.68 & -0.10 & -14.96 & 1 & 0.41 & 13986 & 0.40 & 0.26 & 0.63 & -15.20 & 0.16 \\
\hline TOT & $\mathrm{CE}$ & 0.55 & 0.69 & -0.14 & -19.73 & 1 & 0.40 & 55167 & 0.30 & 0.36 & 0.90 & -22.33 & 0.19 \\
\hline DJF & $\mathrm{CM}$ & 0.47 & 0.64 & -0.17 & -25.92 & 1 & 0.29 & 7803 & 0.34 & 0.20 & 0.69 & -29.29 & 0.20 \\
\hline JJA & $\mathrm{CM}$ & 0.14 & 0.30 & -0.16 & -54.86 & 1 & 0.68 & 8262 & -0.04 & 0.60 & 0.87 & -91.85 & 0.19 \\
\hline MAM & $\mathrm{CM}$ & 0.41 & 0.57 & -0.16 & -28.05 & 1 & 0.38 & 8262 & 0.20 & 0.37 & 0.96 & -33.47 & 0.20 \\
\hline SON & $\mathrm{CM}$ & 0.39 & 0.54 & -0.15 & -27.26 & 1 & 0.44 & 8262 & 0.18 & 0.39 & 0.89 & -32.43 & 0.19 \\
\hline TOT & $\mathrm{CM}$ & 0.35 & 0.51 & -0.16 & -31.19 & 1 & 0.78 & 32589 & -0.02 & 0.73 & 0.95 & -47.00 & 0.19 \\
\hline DJF & $\mathrm{EE}$ & 0.67 & 0.80 & -0.14 & -16.84 & 1 & 0.31 & 14637 & 0.53 & 0.17 & 0.54 & -17.99 & 0.16 \\
\hline JJA & $\mathrm{EE}$ & 0.40 & 0.55 & -0.15 & -27.46 & 1 & 0.74 & 15498 & 0.02 & 0.69 & 0.93 & -34.04 & 0.18 \\
\hline MAM & $\mathrm{EE}$ & 0.59 & 0.65 & -0.06 & -9.20 & 1 & 0.34 & 15498 & 0.43 & 0.25 & 0.74 & -9.03 & 0.13 \\
\hline SON & $\mathrm{EE}$ & 0.58 & 0.67 & -0.08 & -12.21 & 1 & 0.76 & 15498 & 0.21 & 0.56 & 0.74 & -12.39 & 0.12 \\
\hline TOT & $\mathrm{EE}$ & 0.56 & 0.67 & -0.11 & -16.00 & 1 & 0.74 & 61131 & 0.11 & 0.67 & 0.91 & -18.37 & 0.15 \\
\hline DJF & EM & 0.49 & 0.66 & -0.17 & -25.67 & 1 & 0.47 & 18819 & 0.22 & 0.40 & 0.86 & -29.59 & 0.22 \\
\hline JJA & $\mathrm{EM}$ & 0.10 & 0.18 & -0.08 & -45.93 & 1 & 0.76 & 19926 & -0.01 & 0.57 & 0.75 & -82.30 & 0.13 \\
\hline MAM & $\mathrm{EM}$ & 0.36 & 0.51 & -0.14 & -28.60 & 1 & 0.73 & 19926 & -0.02 & 0.75 & 1.02 & -38.06 & 0.19 \\
\hline SON & $\mathrm{EM}$ & 0.33 & 0.44 & -0.12 & -26.57 & 1 & 0.73 & 19926 & 0.05 & 0.62 & 0.85 & -33.17 & 0.18 \\
\hline TOT & $\mathrm{EM}$ & 0.32 & 0.44 & -0.13 & -28.83 & 1 & 0.84 & 78597 & 0.00 & 0.71 & 0.85 & -46.01 & 0.18 \\
\hline DJF & $\mathrm{EU}$ & 0.53 & 0.68 & -0.15 & -21.81 & 1 & 0.75 & 127942 & 0.19 & 0.50 & 0.67 & -20.99 & 0.21 \\
\hline JJA & $\mathrm{EU}$ & 0.30 & 0.47 & -0.17 & -36.57 & 1 & 0.87 & 135468 & 0.00 & 0.63 & 0.73 & -50.47 & 0.23 \\
\hline MAM & $\mathrm{EU}$ & 0.47 & 0.59 & -0.12 & -20.53 & 1 & 0.74 & 135468 & 0.12 & 0.59 & 0.80 & -22.29 & 0.19 \\
\hline SON & $\mathrm{EU}$ & 0.47 & 0.59 & -0.13 & -21.14 & 1 & 0.80 & 135468 & 0.13 & 0.57 & 0.72 & -19.80 & 0.19 \\
\hline TOT & $\mathrm{EU}$ & 0.44 & 0.58 & -0.14 & -24.34 & 1 & 0.81 & 534346 & 0.07 & 0.63 & 0.78 & -28.49 & 0.21 \\
\hline DJF & IP & 0.50 & 0.54 & -0.04 & -7.68 & 1 & 0.32 & 6069 & 0.38 & 0.22 & 0.69 & -6.24 & 0.15 \\
\hline JJA & IP & 0.23 & 0.33 & -0.10 & -30.11 & 1 & 0.52 & 6426 & 0.10 & 0.39 & 0.75 & -37.14 & 0.18 \\
\hline MAM & IP & 0.48 & 0.56 & -0.08 & -14.37 & 1 & 0.39 & 6426 & 0.30 & 0.32 & 0.81 & -15.05 & 0.16 \\
\hline SON & IP & 0.45 & 0.49 & -0.04 & -7.95 & 1 & 0.52 & 6426 & 0.25 & 0.41 & 0.79 & -7.08 & 0.14 \\
\hline TOT & IP & 0.41 & 0.48 & -0.07 & -13.65 & 1 & 0.63 & 25347 & 0.14 & 0.56 & 0.90 & -16.52 & 0.16 \\
\hline DJF & LA & 0.54 & 0.61 & -0.07 & -11.53 & 1 & 0.88 & 80767 & 0.16 & 0.62 & 0.71 & -7.73 & 0.14 \\
\hline JJA & LA & 0.31 & 0.43 & -0.12 & -26.92 & 1 & 0.91 & 85518 & 0.01 & 0.70 & 0.78 & -29.04 & 0.16 \\
\hline MAM & LA & 0.48 & 0.54 & -0.05 & -9.82 & 1 & 0.84 & 85518 & 0.10 & 0.71 & 0.85 & -9.07 & 0.12 \\
\hline SON & LA & 0.48 & 0.53 & -0.05 & -9.44 & 1 & 0.88 & 85518 & 0.13 & 0.66 & 0.75 & -3.57 & 0.13 \\
\hline TOT & LA & 0.45 & 0.53 & -0.07 & -13.74 & 1 & 0.88 & 337321 & 0.08 & 0.72 & 0.82 & -12.42 & 0.14 \\
\hline DJF & NA & 0.30 & 0.27 & 0.02 & 8.12 & 1 & 0.53 & 18360 & 0.18 & 0.42 & 0.78 & 11.55 & 0.12 \\
\hline JJA & NA & 0.11 & 0.15 & -0.04 & -29.22 & 1 & 0.71 & 19440 & 0.02 & 0.57 & 0.80 & -16.70 & 0.10 \\
\hline MAM & NA & 0.26 & 0.26 & -0.01 & -2.27 & 1 & 0.63 & 19440 & 0.10 & 0.58 & 0.91 & -1.23 & 0.10 \\
\hline SON & NA & 0.28 & 0.25 & 0.03 & 13.14 & 1 & 0.73 & 19440 & 0.10 & 0.71 & 0.98 & 20.29 & 0.11 \\
\hline TOT & NA & 0.23 & 0.23 & 0.00 & 0.37 & 0 & 0.70 & 76680 & 0.07 & 0.68 & 0.98 & 3.36 & 0.11 \\
\hline DJF & $\mathrm{NE}$ & 0.65 & 0.83 & -0.18 & -21.20 & 1 & 0.00 & 15691 & 0.65 & 0.00 & 0.54 & -23.25 & 0.20 \\
\hline JJA & $\mathrm{NE}$ & 0.52 & 0.70 & -0.18 & -25.63 & 1 & 0.33 & 16614 & 0.33 & 0.27 & 0.82 & -29.39 & 0.21 \\
\hline MAM & $\mathrm{NE}$ & 0.59 & 0.68 & -0.09 & -12.79 & 1 & 0.29 & 16614 & 0.46 & 0.20 & 0.69 & -13.12 & 0.14 \\
\hline SON & $\mathrm{NE}$ & 0.62 & 0.78 & -0.17 & -21.33 & 1 & 0.44 & 16614 & 0.40 & 0.28 & 0.65 & -23.40 & 0.20 \\
\hline TOT & $\mathrm{NE}$ & 0.59 & 0.75 & -0.15 & -20.34 & 1 & 0.43 & 65533 & 0.36 & 0.31 & 0.72 & -22.28 & 0.19 \\
\hline DJF & $\mathrm{OC}$ & 0.52 & 0.80 & -0.28 & -35.23 & 1 & 0.76 & 47175 & 0.00 & 0.64 & 0.85 & -43.70 & 0.30 \\
\hline JJA & $\mathrm{OC}$ & 0.27 & 0.54 & -0.27 & -49.63 & 1 & 0.91 & 49950 & -0.07 & 0.63 & 0.69 & -87.16 & 0.31 \\
\hline MAM & $\mathrm{OC}$ & 0.44 & 0.68 & -0.24 & -34.98 & 1 & 0.82 & 49950 & -0.02 & 0.68 & 0.82 & -44.91 & 0.26 \\
\hline SON & $\mathrm{OC}$ & 0.44 & 0.70 & -0.25 & -36.48 & 1 & 0.89 & 49950 & -0.03 & 0.68 & 0.77 & -47.60 & 0.27 \\
\hline TOT & $\mathrm{OC}$ & 0.42 & 0.68 & -0.26 & -38.42 & 1 & 0.88 & 197025 & -0.05 & 0.70 & 0.79 & -56.01 & 0.28 \\
\hline
\end{tabular}




\section{Cloud Optical Thickness}

Table S5. The same as Table S3 but for RegCM4 and CM SAF COT.

\begin{tabular}{|c|c|c|c|c|c|c|c|c|c|c|c|c|c|c|c|}
\hline & \multicolumn{3}{|c|}{$\mathrm{ANN}$} & \multicolumn{3}{|c|}{ DJF } & \multicolumn{3}{|c|}{ MAM } & \multicolumn{3}{|c|}{ JJA } & \multicolumn{3}{|c|}{$\mathrm{SON}$} \\
\hline & MOD & SAT & bias & MOD & SAT & bias & MOD & SAT & bias & MOD & SAT & bias & MOD & SAT & bias \\
\hline $\mathrm{CE}$ & $14.63 \pm 5.76$ & $11.79 \pm 3.80$ & 24.09 & $16.75 \pm 5.9$ & $13.45 \pm 4.87$ & 24.56 & $16.1 \pm 4.72$ & $10.41 \pm 2.97$ & 54.58 & $10.35 \pm 4.35$ & $10.87 \pm 2.65$ & -4.82 & $15.79 \pm 5.62$ & $12.79 \pm 3.76$ & 23.46 \\
\hline $\mathrm{CM}$ & $6.4 \pm 3.71$ & $9.24 \pm 3.83$ & -30.74 & $8.02 \pm 3.7$ & $11.29 \pm 3.96$ & -28.92 & $7.55 \pm 3.6$ & $9.3 \pm 3.16$ & -18.83 & $3.44 \pm 1.91$ & $6.69 \pm 3.22$ & -48.67 & $6.58 \pm 3.45$ & $9.71 \pm 3.462$ & -32.26 \\
\hline $\mathrm{EE}$ & $15.64 \pm 6.02$ & $11.95 \pm 4.01$ & 30.85 & $18.1 \pm 4.32$ & $14.23 \pm 4.89$ & 27.17 & $16.72 \pm 4.97$ & $10.01 \pm 2.70$ & 67.02 & $9.9 \pm 3.98$ & $10.61 \pm 2.69$ & -6.67 & $18.36 \pm 6.00$ & $13.44 \pm 3.972$ & 36.60 \\
\hline EM & $7.15 \pm 4.93$ & $9.17 \pm 4.701$ & -21.96 & $9.64 \pm 5.19$ & $11.51 \pm 4.82$ & -16.25 & $7.65 \pm 4.57$ & $8.23 \pm 3.41$ & -7.03 & $3.81 \pm 2.7$ & $7.02 \pm 4.47$ & -45.75 & $7.04 \pm 4.89$ & $9.64 \pm 4.812$ & -27.02 \\
\hline $\mathrm{EU}$ & $10.05 \pm 6.4$ & $9.63 \pm 4.22$ & 4.29 & $10.78 \pm 6.05$ & $11.04 \pm 4.89$ & -2.31 & $10.74 \pm 6.1$ & $8.67 \pm 3.38$ & 23.83 & $7.85 \pm 5.76$ & $8.72 \pm 3.48$ & -9.98 & $10.87 \pm 7.05$ & $10.38 \pm 4.581$ & 4.68 \\
\hline IP & $8.29 \pm 4.98$ & $9.63 \pm 3.59$ & -13.85 & $11.01 \pm 5.25$ & $11.13 \pm 3.90$ & $-1.08^{*}$ & $9.03 \pm 4.4$ & $9.4 \pm 2.94$ & -3.88 & $4.51 \pm 2.32$ & $8.01 \pm 3.09$ & -43.71 & $8.77 \pm 5.00$ & $10.06 \pm 3.634$ & -12.78 \\
\hline LA & $11.45 \pm 6.96$ & $10.67 \pm 4.40$ & 7.29 & $12.63 \pm 6.49$ & $12.01 \pm 5.28$ & 5.22 & $12.21 \pm 6.64$ & $9.62 \pm 3.58$ & 27.02 & $8.34 \pm 6.01$ & $9.81 \pm 3.42$ & -14.94 & $12.69 \pm 7.60$ & $11.49_{ \pm} .725$ & 10.41 \\
\hline NA & $4.77 \pm 2.93$ & $7.93 \pm 3.43$ & -39.81 & $6.22 \pm 3.2$ & $8.25 \pm 3.36$ & -24.68 & $4.81 \pm 2.48$ & $7.65 \pm 3.14$ & -37.11 & $2.88 \pm 1.8$ & $7.97 \pm 3.65$ & -63.83 & $4.92 \pm 2.98$ & $7.88 \pm 3.581$ & -37.57 \\
\hline $\mathrm{NE}$ & $15.24 \pm 5.6$ & $9.88 \pm 5.22$ & 54.33 & $12.81 \pm 3.78$ & $11.34 \pm 7.36$ & 12.97 & $15.11 \pm 4.87$ & $8.64 \pm 4.95$ & 74.84 & $14.57 \pm 5.68$ & $9.16 \pm 2.87$ & 59.04 & $17.34 \pm 6.30$ & $11.83 \pm 6.081$ & 46.63 \\
\hline $\mathrm{OC}$ & $7.71_{ \pm 4.43}$ & $7.9 \pm 3.23$ & -2.46 & $7.55 \pm 3.23$ & $9.35 \pm 3.54$ & -19.22 & $8.33 \pm 4.06$ & $7.13 \pm 2.32$ & 16.83 & $7.04 \pm 5.24$ & $6.93 \pm 2.78$ & 1.46 & $7.84 \pm 4.66$ & $8.54 \pm 3.646$ & -8.17 \\
\hline
\end{tabular}


Table S6. Average RegCM4 COT, CM SAF COT (MSG SEVIRI), their difference, the corresponding NMB and the statistical significance of the results at the $95 \%$ confidence level due to a two independent sample t-test per season and region. The NSD, MNMB and RMSE are also given along with Pearson's correlation coefficient $(\mathrm{R})$, the number of observations used and the coefficients $a$ and $b$ of the regression line $(\mathrm{RegCM} 4=\mathrm{a} \cdot \mathrm{CMSAF}+\mathrm{b})$. The region are listed in alphabetic order.

\begin{tabular}{|c|c|c|c|c|c|c|c|c|c|c|c|c|c|}
\hline Season & Region & REG & CMS & Diff. & NMB & Signif. & $\mathrm{R}$ & Obs. & $\mathrm{b}$ & $\mathrm{a}$ & NSD & MNMB & RMSE \\
\hline DJF & $\mathrm{CE}$ & 16.75 & 13.45 & 3.30 & 24.56 & 1 & 0.18 & 10956 & 13.83 & 0.22 & 1.21 & 20.51 & 7.69 \\
\hline JJA & $\mathrm{CE}$ & 10.35 & 10.87 & -0.52 & -4.82 & 1 & 0.37 & 13986 & 3.78 & 0.60 & 1.64 & -10.97 & 4.21 \\
\hline MAM & $\mathrm{CE}$ & 16.10 & 10.41 & 5.68 & 54.58 & 1 & 0.26 & 13986 & 11.80 & 0.41 & 1.59 & 41.43 & 7.49 \\
\hline SON & $\mathrm{CE}$ & 15.79 & 12.79 & 3.00 & 23.46 & 1 & 0.30 & 13986 & 10.00 & 0.45 & 1.50 & 17.56 & 6.48 \\
\hline TOT & $\mathrm{CE}$ & 14.63 & 11.79 & 2.84 & 24.09 & 1 & 0.29 & 52914 & 9.40 & 0.44 & 1.52 & 16.94 & 6.54 \\
\hline DJF & $\mathrm{CM}$ & 8.02 & 11.29 & -3.26 & -28.92 & 1 & 0.43 & 7803 & 3.46 & 0.40 & 0.93 & -35.88 & 5.23 \\
\hline JJA & $\mathrm{CM}$ & 3.44 & 6.69 & -3.26 & -48.67 & 1 & 0.31 & 7948 & 2.22 & 0.18 & 0.59 & -62.84 & 4.57 \\
\hline MAM & $\mathrm{CM}$ & 7.55 & 9.30 & -1.75 & -18.83 & 1 & 0.49 & 8262 & 2.39 & 0.55 & 1.14 & -24.17 & 3.86 \\
\hline $\mathrm{SON}$ & $\mathrm{CM}$ & 6.58 & 9.71 & -3.13 & -32.26 & 1 & 0.32 & 8262 & 3.46 & 0.32 & 1.00 & -41.64 & 5.10 \\
\hline TOT & $\mathrm{CM}$ & 6.40 & 9.24 & -2.84 & -30.74 & 1 & 0.50 & 32275 & 1.92 & 0.49 & 0.97 & -41.00 & 4.72 \\
\hline DJF & $\mathrm{EE}$ & 18.10 & 14.23 & 3.87 & 27.17 & 1 & 0.15 & 12139 & 16.18 & 0.13 & 0.88 & 25.69 & 7.14 \\
\hline JJA & $\mathrm{EE}$ & 9.90 & 10.61 & -0.71 & -6.67 & 1 & 0.22 & 15475 & 6.46 & 0.32 & 1.48 & -11.41 & 4.34 \\
\hline MAM & $\mathrm{EE}$ & 16.72 & 10.01 & 6.71 & 67.02 & 1 & 0.33 & 15470 & 10.70 & 0.60 & 1.84 & 47.56 & 8.26 \\
\hline SON & $\mathrm{EE}$ & 18.36 & 13.44 & 4.92 & 36.60 & 1 & 0.36 & 15477 & 11.04 & 0.54 & 1.51 & 28.42 & 7.67 \\
\hline TOT & $\mathrm{EE}$ & 15.64 & 11.95 & 3.69 & 30.85 & 1 & 0.35 & 58561 & 9.38 & 0.52 & 1.50 & 22.39 & 7.01 \\
\hline DJF & EM & 9.64 & 11.51 & -1.87 & -16.25 & 1 & 0.48 & 18819 & 3.74 & 0.51 & 1.08 & -22.96 & 5.46 \\
\hline JJA & EM & 3.81 & 7.02 & -3.21 & -45.75 & 1 & 0.52 & 16268 & 1.62 & 0.31 & 0.60 & -53.52 & 5.01 \\
\hline MAM & EM & 7.65 & 8.23 & -0.58 & -7.03 & 1 & 0.60 & 19926 & 1.08 & 0.80 & 1.34 & -14.00 & 3.78 \\
\hline SON & EM & 7.04 & 9.64 & -2.61 & -27.03 & 1 & 0.61 & 19842 & 1.11 & 0.61 & 1.02 & -37.93 & 5.04 \\
\hline TOT & $\mathrm{EM}$ & 7.15 & 9.17 & -2.01 & -21.96 & 1 & 0.58 & 74855 & 1.56 & 0.61 & 1.05 & -31.19 & 4.85 \\
\hline DJF & $\mathrm{EU}$ & 10.78 & 11.04 & -0.26 & -2.31 & 1 & 0.47 & 101916 & 4.31 & 0.59 & 1.24 & -9.40 & 5.70 \\
\hline JJA & $\mathrm{EU}$ & 7.85 & 8.72 & -0.87 & -9.98 & 1 & 0.40 & 121638 & 2.13 & 0.66 & 1.65 & -27.80 & 5.49 \\
\hline MAM & $\mathrm{EU}$ & 10.74 & 8.67 & 2.07 & 23.83 & 1 & 0.39 & 130152 & 4.64 & 0.70 & 1.80 & 9.07 & 6.07 \\
\hline SON & $\mathrm{EU}$ & 10.87 & 10.38 & 0.49 & 4.68 & 1 & 0.54 & 123815 & 2.28 & 0.83 & 1.54 & -9.27 & 6.02 \\
\hline TOT & $\mathrm{EU}$ & 10.05 & 9.63 & 0.41 & 4.29 & 1 & 0.46 & 477521 & 3.32 & 0.70 & 1.52 & -9.02 & 5.84 \\
\hline DJF & IP & 11.01 & 11.13 & -0.12 & -1.08 & 0 & 0.41 & 6069 & 4.82 & 0.56 & 1.35 & -5.86 & 5.09 \\
\hline JJA & IP & 4.51 & 8.01 & -3.50 & -43.71 & 1 & 0.44 & 6419 & 1.87 & 0.33 & 0.75 & -57.96 & 4.57 \\
\hline MAM & IP & 9.03 & 9.40 & -0.36 & -3.88 & 1 & 0.39 & 6426 & 3.57 & 0.58 & 1.50 & -9.86 & 4.26 \\
\hline SON & IP & 8.77 & 10.06 & -1.29 & -12.79 & 1 & 0.54 & 6426 & 1.37 & 0.74 & 1.37 & -22.29 & 4.51 \\
\hline TOT & IP & 8.29 & 9.63 & -1.33 & -13.85 & 1 & 0.51 & 25340 & 1.48 & 0.71 & 1.39 & -24.24 & 4.61 \\
\hline DJF & LA & 12.63 & 12.01 & 0.63 & 5.22 & 1 & 0.45 & 64836 & 6.01 & 0.55 & 1.23 & -1.69 & 6.30 \\
\hline JJA & LA & 8.34 & 9.81 & -1.47 & -14.94 & 1 & 0.33 & 75424 & 2.75 & 0.57 & 1.76 & -33.69 & 6.05 \\
\hline MAM & LA & 12.21 & 9.62 & 2.60 & 27.02 & 1 & 0.35 & 80657 & 5.97 & 0.65 & 1.86 & 10.77 & 6.86 \\
\hline $\mathrm{SON}$ & LA & 12.69 & 11.49 & 1.20 & 10.40 & 1 & 0.51 & 77414 & 3.29 & 0.82 & 1.61 & -4.01 & 6.71 \\
\hline TOT & LA & 11.45 & 10.67 & 0.78 & 7.29 & 1 & 0.43 & 298331 & 4.23 & 0.68 & 1.58 & -7.01 & 6.50 \\
\hline DJF & NA & 6.22 & 8.25 & -2.04 & -24.68 & 1 & 0.40 & 18240 & 3.07 & 0.38 & 0.95 & -31.11 & 4.13 \\
\hline JJA & NA & 2.88 & 7.97 & -5.09 & -63.83 & 1 & 0.32 & 15742 & 1.63 & 0.16 & 0.49 & -93.43 & 6.19 \\
\hline MAM & NA & 4.81 & 7.65 & -2.84 & -37.11 & 1 & 0.37 & 19429 & 2.57 & 0.29 & 0.79 & -46.61 & 4.28 \\
\hline SON & NA & 4.92 & 7.88 & -2.96 & -37.57 & 1 & 0.42 & 18637 & 2.16 & 0.35 & 0.83 & -49.00 & 4.63 \\
\hline TOT & NA & 4.77 & 7.93 & -3.16 & -39.82 & 1 & 0.36 & 72048 & 2.37 & 0.30 & 0.85 & -53.53 & 4.81 \\
\hline DJF & $\mathrm{NE}$ & 12.81 & 11.34 & 1.47 & 12.97 & 1 & 0.25 & 4014 & 11.34 & 0.13 & 0.51 & 21.54 & 7.52 \\
\hline JJA & $\mathrm{NE}$ & 14.57 & 9.16 & 5.41 & 59.04 & 1 & 0.32 & 12484 & 8.77 & 0.63 & 1.98 & 41.88 & 7.70 \\
\hline MAM & $\mathrm{NE}$ & 15.11 & 8.64 & 6.47 & 74.84 & 1 & 0.23 & 12453 & 13.18 & 0.22 & 0.98 & 56.75 & 8.89 \\
\hline SON & $\mathrm{NE}$ & 17.34 & 11.83 & 5.52 & 46.63 & 1 & 0.31 & 9471 & 13.58 & 0.32 & 1.04 & 40.13 & 9.14 \\
\hline TOT & $\mathrm{NE}$ & 15.24 & 9.88 & 5.37 & 54.34 & 1 & 0.27 & 38422 & 12.36 & 0.29 & 1.07 & 44.14 & 8.45 \\
\hline DJF & $\mathrm{OC}$ & 7.55 & 9.35 & -1.80 & -19.22 & 1 & 0.27 & 37080 & 5.25 & 0.25 & 0.91 & -22.89 & 4.47 \\
\hline JJA & $\mathrm{OC}$ & 7.04 & 6.93 & 0.10 & 1.47 & 1 & 0.53 & 46214 & 0.10 & 1.00 & 1.89 & -18.19 & 4.44 \\
\hline MAM & $\mathrm{OC}$ & 8.33 & 7.13 & 1.20 & 16.83 & 1 & 0.17 & 49495 & 6.19 & 0.30 & 1.75 & 6.30 & 4.48 \\
\hline SON & $\mathrm{OC}$ & 7.84 & 8.54 & -0.70 & -8.17 & 1 & 0.41 & 46401 & 3.42 & 0.52 & 1.28 & -18.06 & 4.66 \\
\hline TOT & $\mathrm{OC}$ & 7.71 & 7.90 & -0.19 & -2.46 & 1 & 0.34 & 179190 & 4.03 & 0.47 & 1.37 & -12.36 & 4.52 \\
\hline
\end{tabular}




\section{Effective Radius (liquid clouds)}

a)

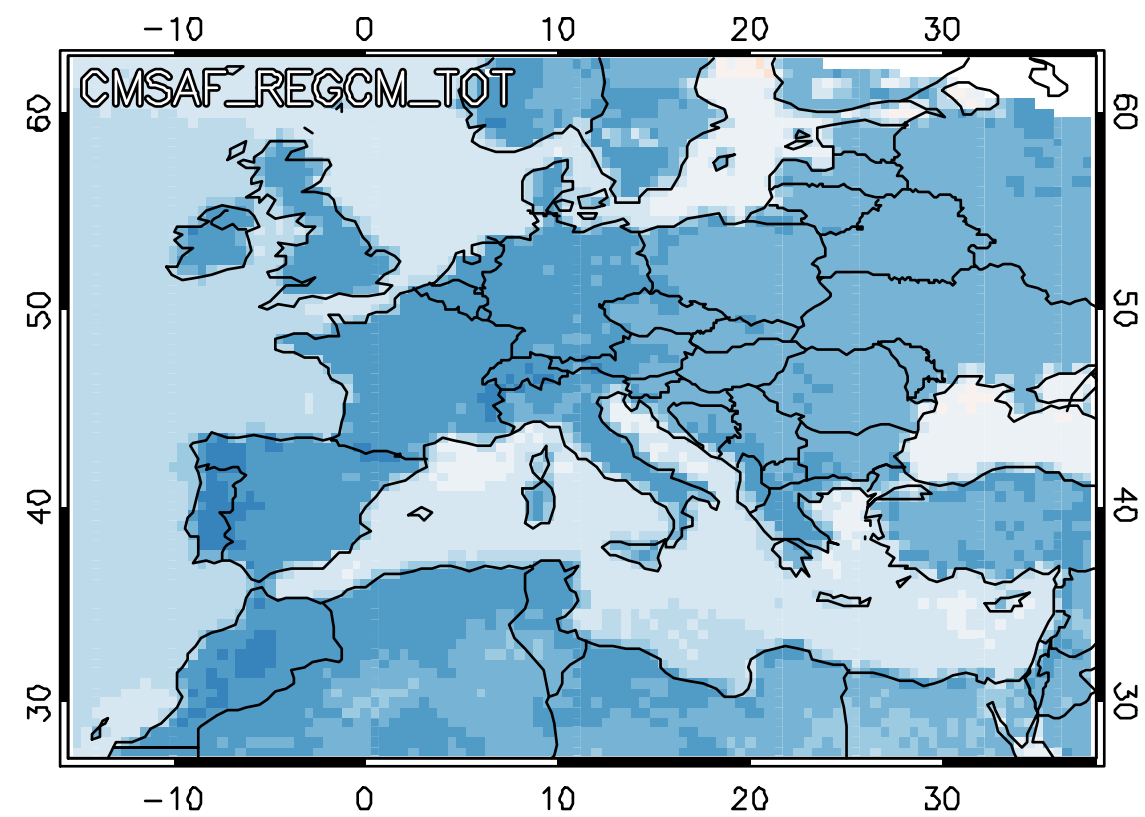

b)

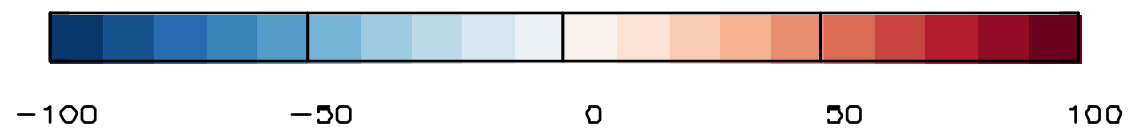

\section{EUROPE_CMSAF_REGCM_TOT}

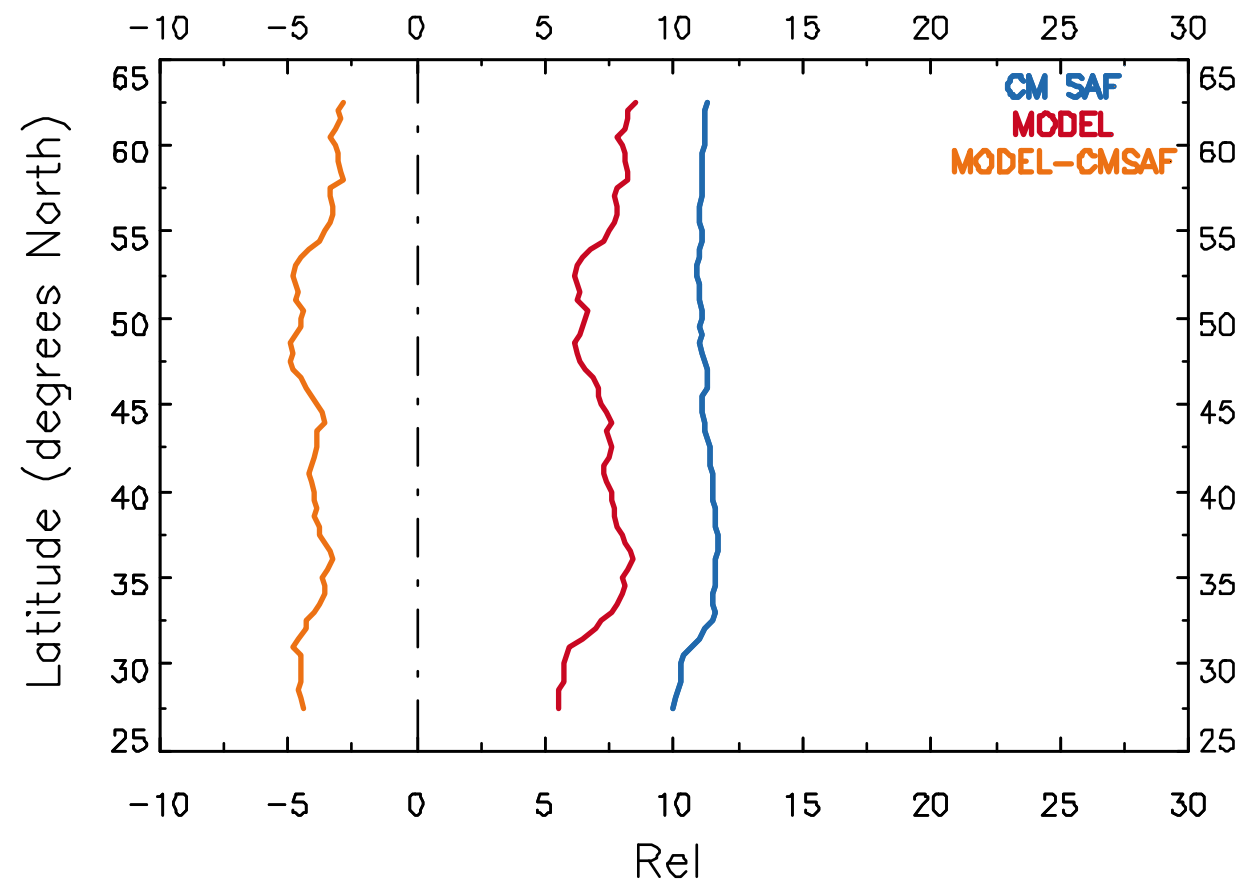

Figure S6. (a) NMB patterns of RegCM4-CM SAF Rel and (b) Latitudinal variability of RegCM4 Rel (red), CM SAF Rel (blue) and their difference (orange) over Europe from MSG SEVIRI observations. 
a)

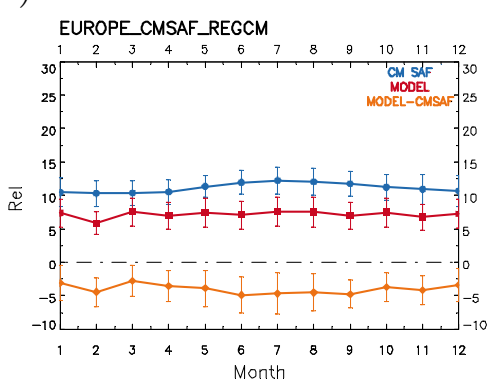

d)

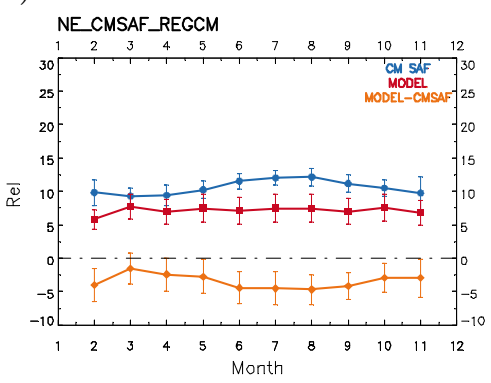

g)

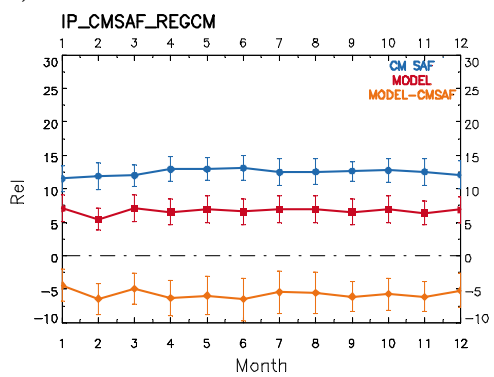

j)

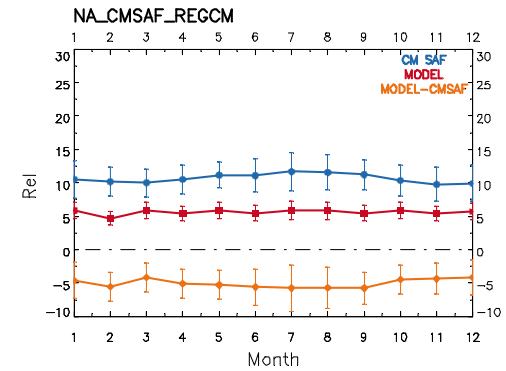

b)

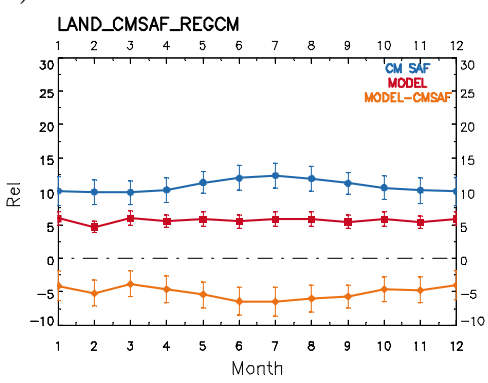

e)

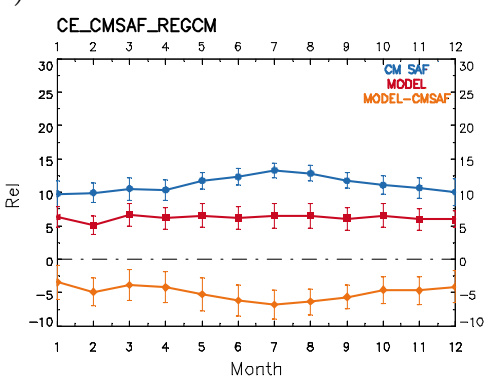

h)

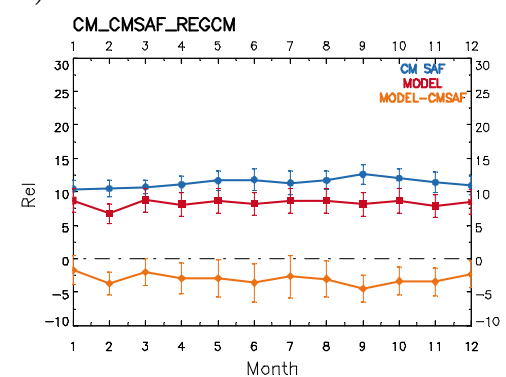

c)

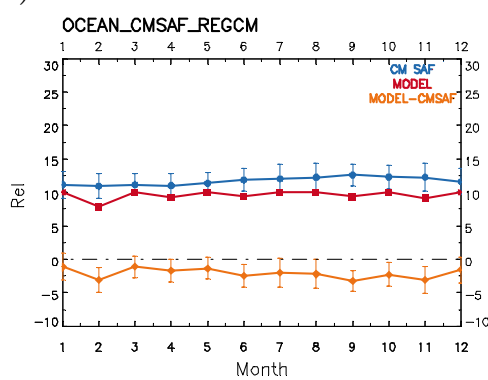

f)

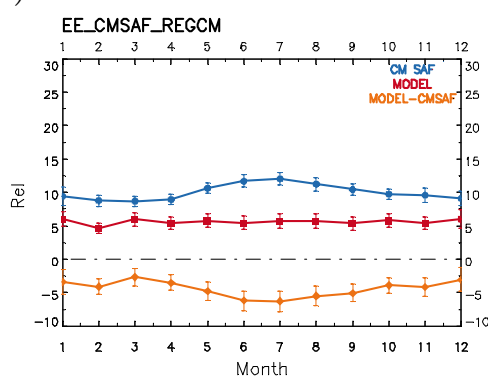

i)

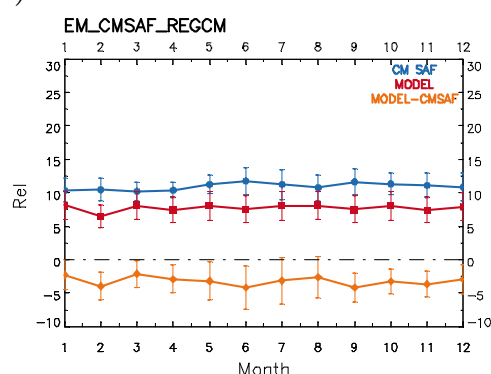

Figure S7. Seasonal variability of RegCM4 Rel (red), CM SAF Rel (blue) and their difference (orange) over (a) the whole Europe, (b) Land, (c) Ocean, (d) NE, (e) CE, (f) EE, (g) IP, (h) CM, (i) EM, (j) NA from MSG SEVIRI observations. 
Table S7. Average RegCM4 Rel, CM SAF Rel (MSG SEVIRI), their difference, the corresponding NMB and the statistical significance of the results at the $95 \%$ confidence level due to a two independent sample t-test for each region of interest. The NSD, MNMB and the RMSE are also given along with Pearson's correlation coefficient (R), the number of observations used and the coefficients $a$ and $b$ of the regression line $(\mathrm{RegCM} 4=\mathrm{a} \cdot \mathrm{CMSAF}+\mathrm{b})$. The region are listed in alphabetic order.

\begin{tabular}{|c|c|c|c|c|c|c|c|c|c|c|c|c|c|}
\hline Season & Region & REG & CMS & Diff. & NMB & Signif. & $\mathrm{R}$ & Obs. & $\mathrm{b}$ & $\mathrm{a}$ & NSD & MNMB & RMSE \\
\hline TOT & $\mathrm{CE}$ & 6.19 & 11.27 & -5.08 & -45.05 & 1 & 0.07 & 35197 & 5.45 & 0.07 & 0.93 & -58.89 & 5.62 \\
\hline TOT & $\mathrm{CM}$ & 8.31 & 11.34 & -3.03 & -26.71 & 1 & -0.03 & 22030 & 8.68 & -0.03 & 1.18 & -32.03 & 3.91 \\
\hline TOT & $\mathrm{EE}$ & 5.58 & 10.06 & -4.48 & -44.55 & 1 & 0.01 & 38829 & 5.54 & 0.00 & 0.74 & -57.15 & 4.83 \\
\hline TOT & EM & 7.73 & 10.93 & -3.20 & -29.25 & 1 & 0.15 & 51998 & 5.91 & 0.17 & 1.13 & -35.82 & 4.10 \\
\hline TOT & $\mathrm{EU}$ & 7.13 & 11.15 & -4.02 & -36.06 & 1 & 0.24 & 321501 & 4.34 & 0.25 & 1.03 & -45.45 & 4.77 \\
\hline TOT & IP & 6.70 & 12.43 & -5.73 & -46.10 & 1 & 0.05 & 17136 & 6.09 & 0.05 & 1.04 & -61.25 & 6.33 \\
\hline TOT & LA & 5.65 & 10.82 & -5.16 & -47.73 & 1 & 0.13 & 196563 & 4.95 & 0.07 & 0.52 & -61.61 & 5.60 \\
\hline TOT & NA & 5.58 & 10.59 & -5.01 & -47.31 & 1 & 0.18 & 48288 & 4.65 & 0.09 & 0.49 & -59.90 & 5.63 \\
\hline TOT & $\mathrm{NE}$ & 7.17 & 10.66 & -3.49 & -32.76 & 1 & 0.06 & 25573 & 6.47 & 0.07 & 1.15 & -40.71 & 4.35 \\
\hline TOT & $\mathrm{OC}$ & 9.56 & 11.71 & -2.15 & -18.33 & 1 & 0.10 & 120242 & 9.17 & 0.03 & 0.34 & -18.93 & 2.92 \\
\hline
\end{tabular}




\section{Effective Radius (ice clouds)}

a)
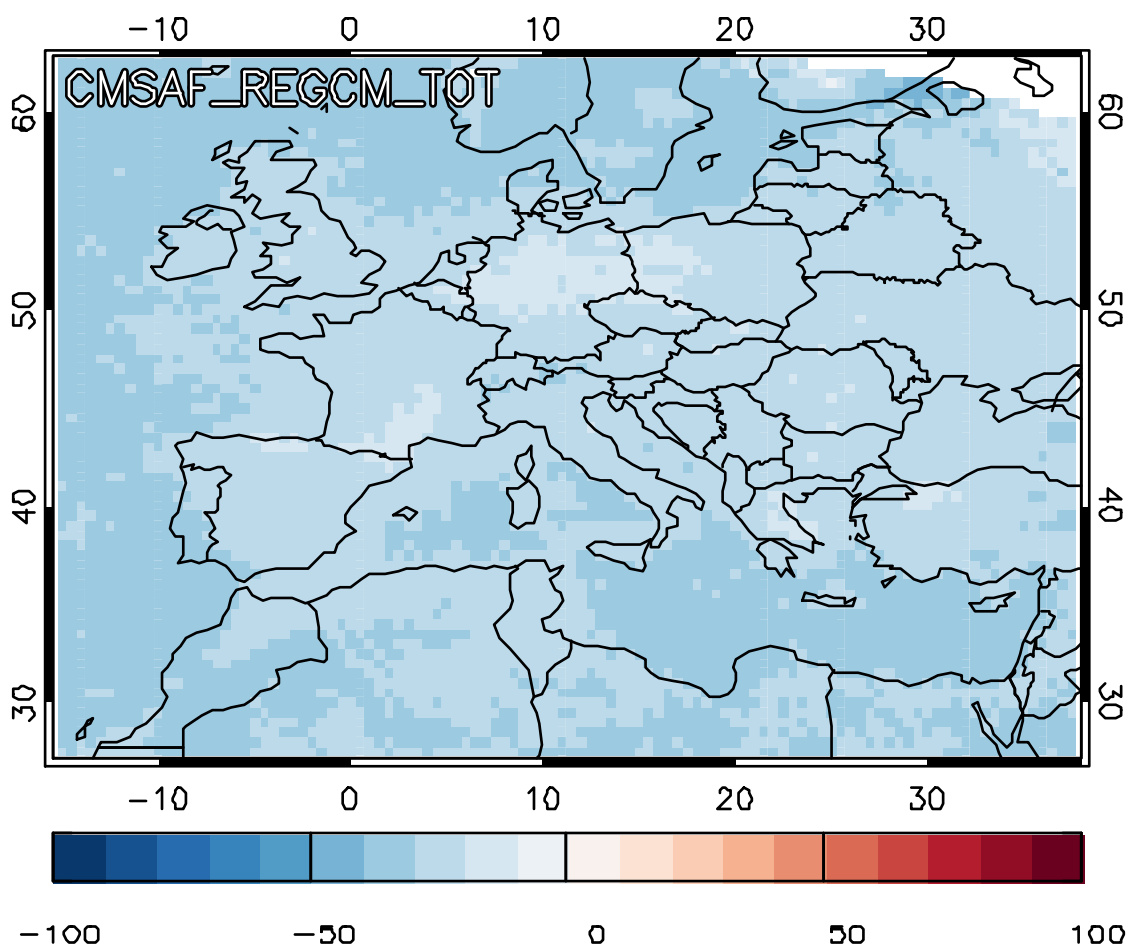

b)

\section{EUROPE_CMSAF_REGCM_TOT}

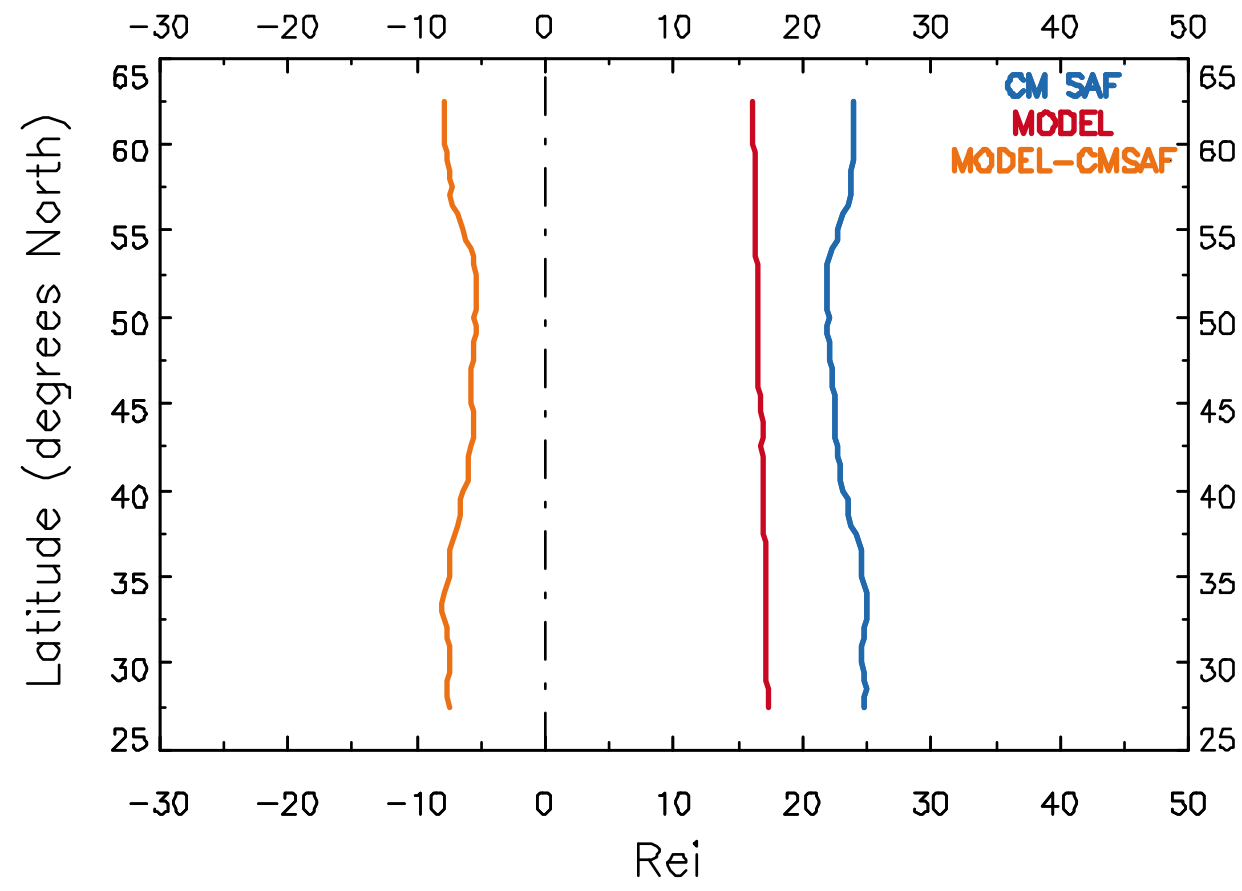

Figure S8. (a) NMB patterns of RegCM4-CM SAF Rei and (b) Latitudinal variability of RegCM4 Rei (red), CM SAF Rei (blue) and their difference (orange) over Europe from MSG SEVIRI observations. 
a)

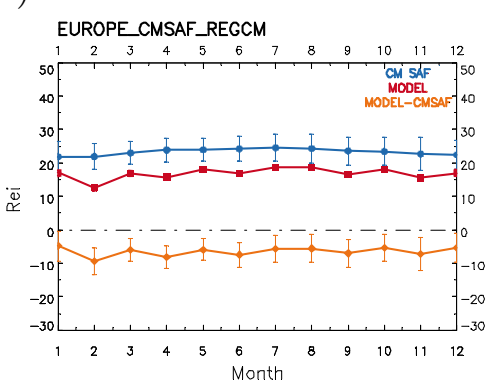

d)

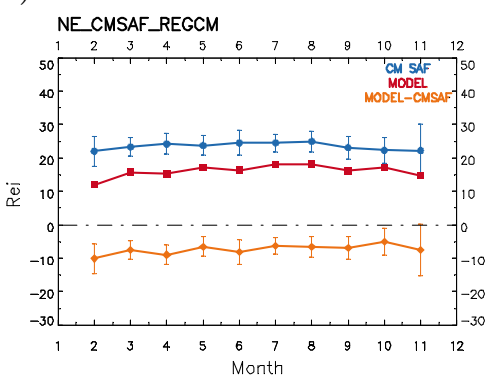

g)

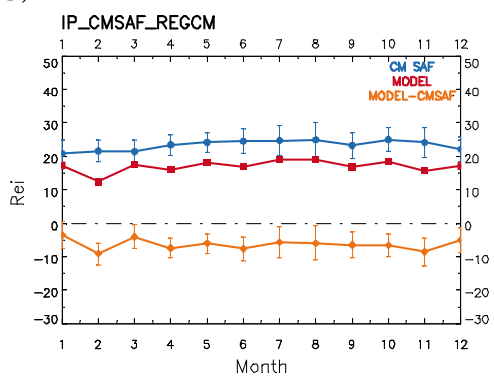

j)

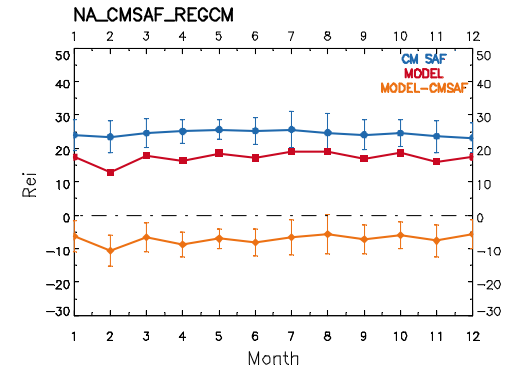

b)

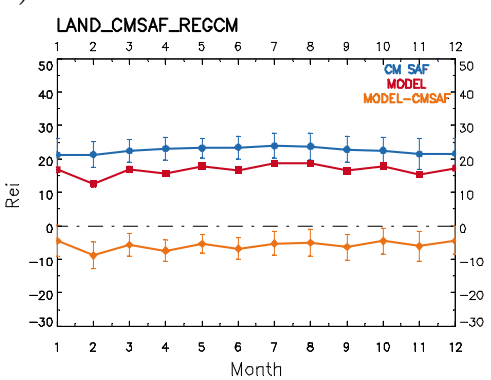

e)

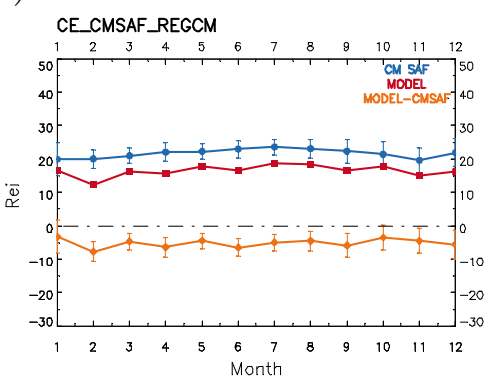

h)

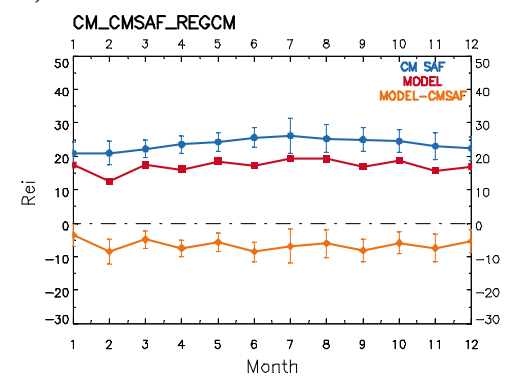

c)

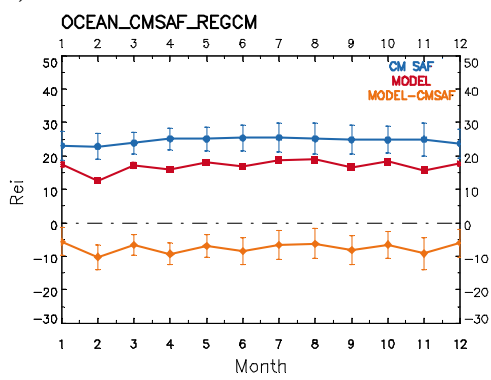

f)

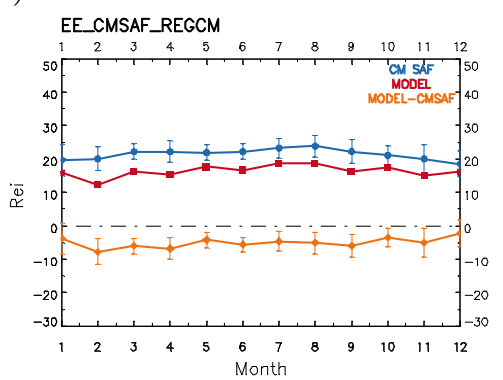

i)

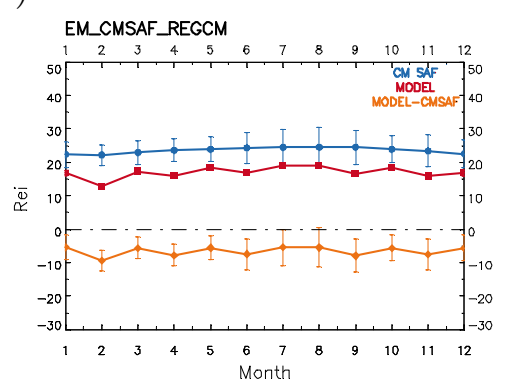

Figure S9. Seasonal variability of RegCM4 Rei (red), CM SAF Rei (blue) and their difference (orange) over (a) the whole Europe, (b) Land, (c) Ocean, (d) NE, (e) CE, (f) EE, (g) IP, (h) CM, (i) EM, (j) NA from MSG SEVIRI observations. 
Table S8. Average RegCM4 Rei, CM SAF Rei (MSG SEVIRI), their difference, the corresponding NMB and the statistical significance of the results at the $95 \%$ confidence level due to a two independent sample t-test for each region of interest. The NSD, MNMB and the RMSE are also given along with Pearson's correlation coefficient (R), the number of observations used and the coefficients $a$ and $b$ of the regression line $(\mathrm{RegCM} 4=\mathrm{a} \cdot \mathrm{CMSAF}+\mathrm{b})$. The region are listed in alphabetic order.

\begin{tabular}{|c|c|c|c|c|c|c|c|c|c|c|c|c|c|}
\hline Season & Region & REG & CMS & Diff. & NMB & Signif. & $\mathrm{R}$ & Obs. & $\mathrm{b}$ & $\mathrm{a}$ & NSD & MNMB & RMSE \\
\hline TOT & $\mathrm{CE}$ & 16.48 & 21.68 & -5.20 & -23.97 & 1 & 0.25 & 35118 & 13.73 & 0.13 & 0.51 & -26.34 & 6.24 \\
\hline TOT & $\mathrm{CM}$ & 17.11 & 23.63 & -6.51 & -27.57 & 1 & 0.29 & 21923 & 13.94 & 0.13 & 0.46 & -30.99 & 7.55 \\
\hline TOT & $\mathrm{EE}$ & 16.36 & 21.57 & -5.22 & -24.18 & 1 & 0.23 & 38976 & 13.86 & 0.12 & 0.49 & -26.49 & 6.33 \\
\hline TOT & EM & 16.77 & 23.42 & -6.65 & -28.40 & 1 & 0.21 & 46496 & 14.83 & 0.08 & 0.40 & -31.73 & 7.89 \\
\hline TOT & $\mathrm{EU}$ & 16.73 & 23.31 & -6.59 & -28.25 & 1 & 0.20 & 311416 & 14.68 & 0.09 & 0.44 & -31.64 & 7.78 \\
\hline TOT & IP & 16.95 & 23.31 & -6.36 & -27.29 & 1 & 0.22 & 16968 & 14.71 & 0.10 & 0.44 & -30.40 & 7.58 \\
\hline TOT & LA & 16.63 & 22.59 & -5.96 & -26.38 & 1 & 0.21 & 191686 & 14.53 & 0.09 & 0.45 & -29.19 & 7.18 \\
\hline TOT & NA & 17.08 & 24.39 & -7.31 & -29.97 & 1 & 0.12 & 45444 & 15.92 & 0.05 & 0.39 & -33.72 & 8.63 \\
\hline TOT & $\mathrm{NE}$ & 16.21 & 23.58 & -7.37 & -31.26 & 1 & 0.17 & 25607 & 14.22 & 0.08 & 0.51 & -36.27 & 8.28 \\
\hline TOT & $\mathrm{OC}$ & 16.93 & 24.55 & -7.62 & -31.05 & 1 & 0.16 & 115049 & 15.21 & 0.07 & 0.45 & -35.68 & 8.70 \\
\hline
\end{tabular}




\section{Aerosol Optical Depth}

a)

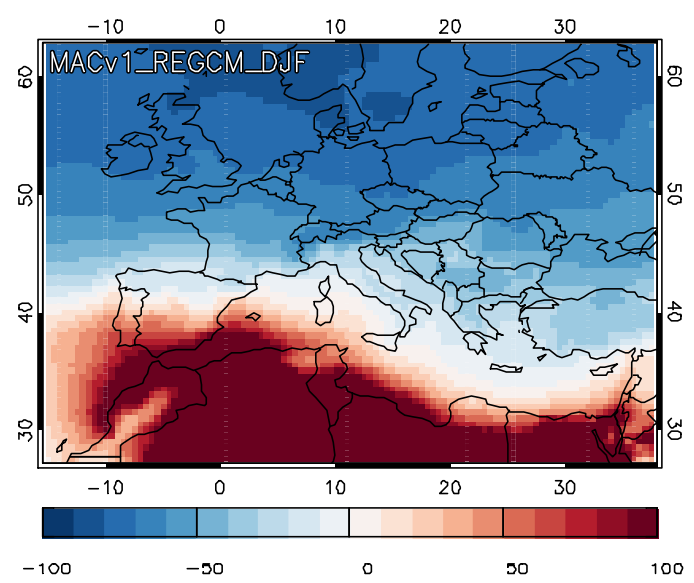

c)

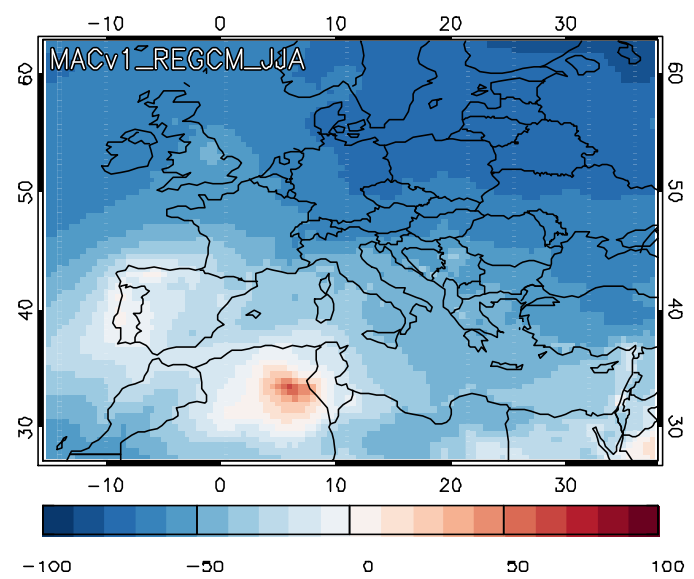

b)

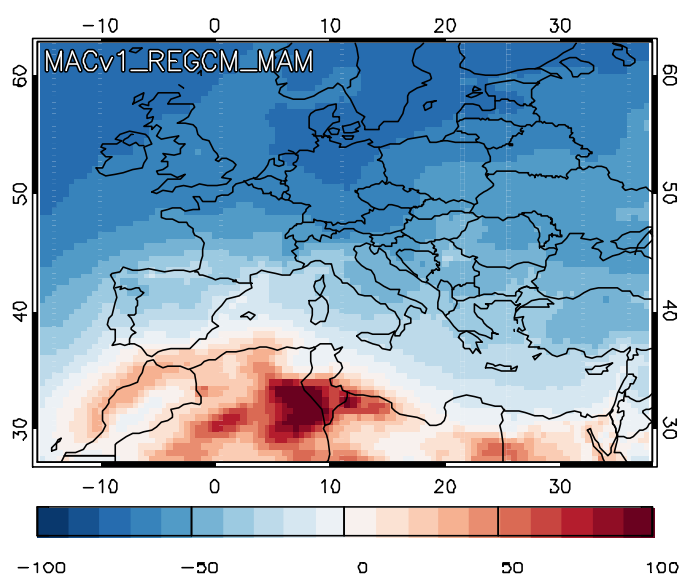

d)

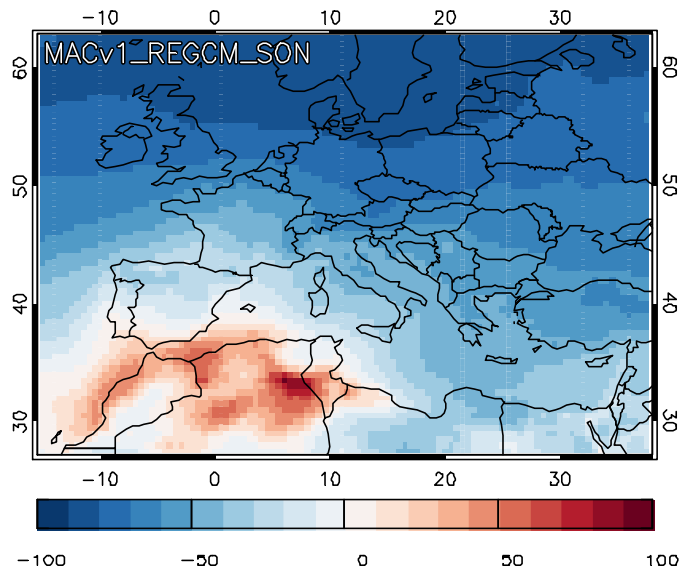

Figure S10. Seasonal Normalized mean bias (NMB) patterns of RegCM4-MACv1 AOD f50 over Europe for (a) winter (DJF), (b) spring (MAM), (c) summer (JJA) and (d) autumn $(\mathrm{SON})$. 
a)

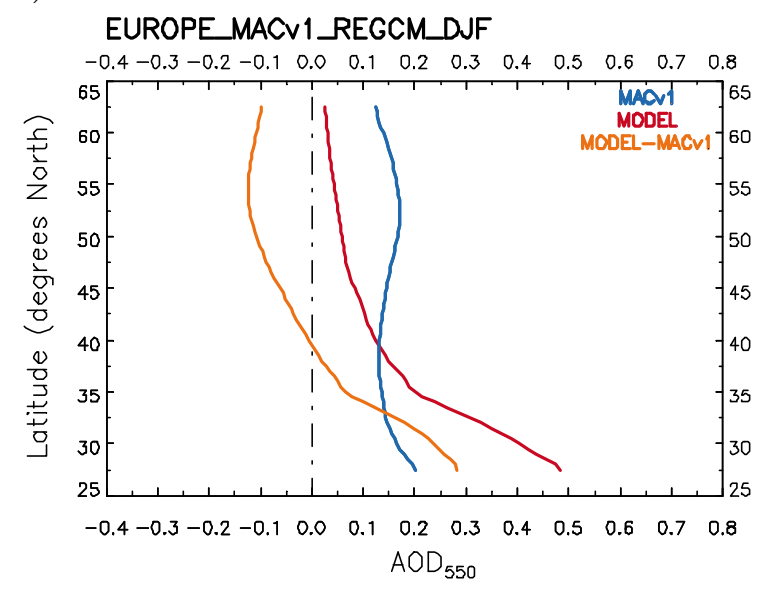

c)

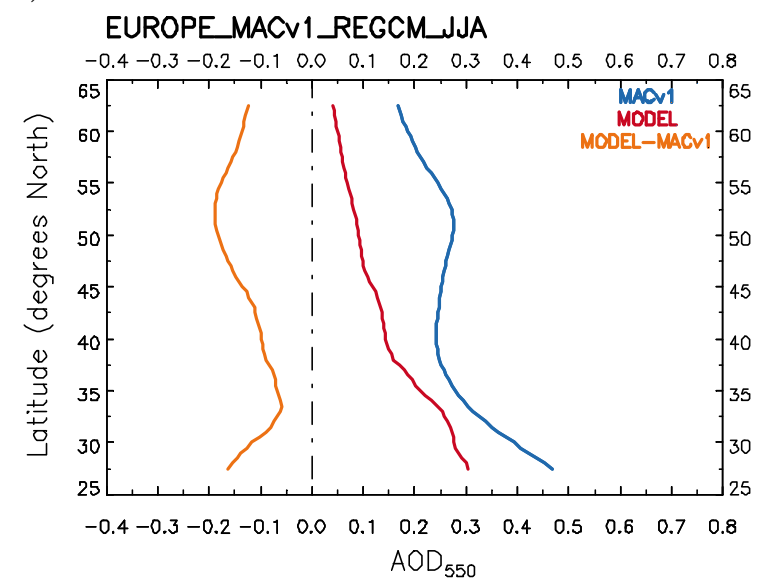

b)

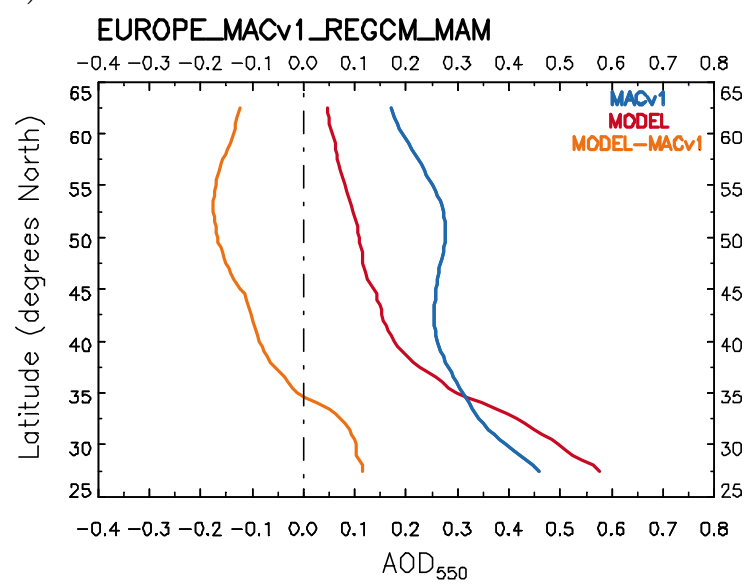

d)

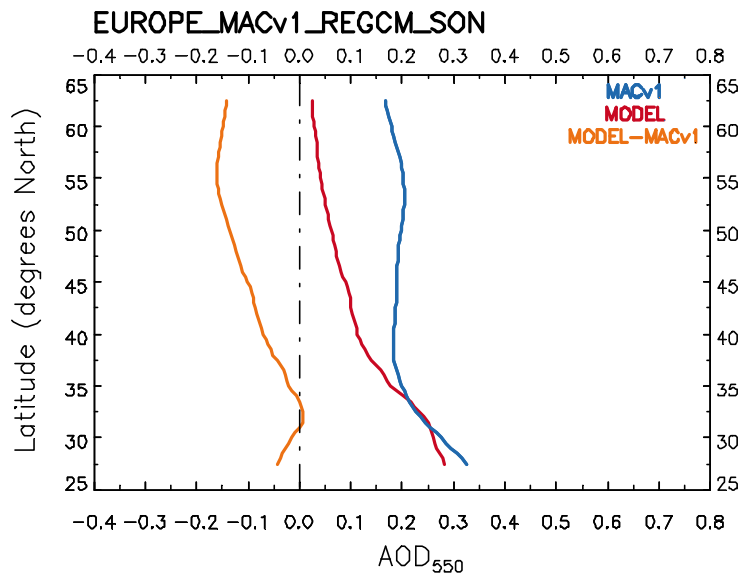

Figure S11. (a) NMB patterns of RegCM4-MACv1 $\mathrm{AOD}_{550}$ and (b) Latitudinal variability of RegCM4 $\mathrm{AOD}_{550}$ (red), MACv1 $\mathrm{AOD}_{550}$ (blue) and their difference (orange) over Europe. 
a)

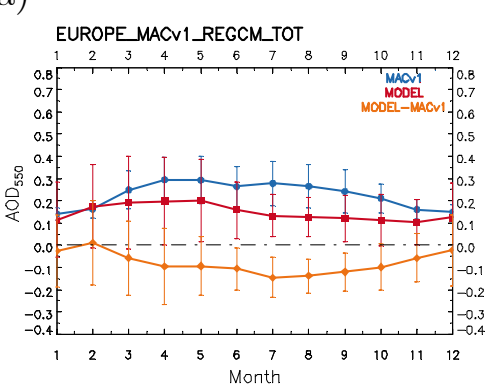

d)

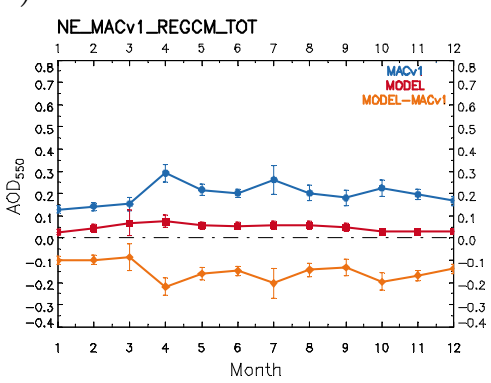

g)

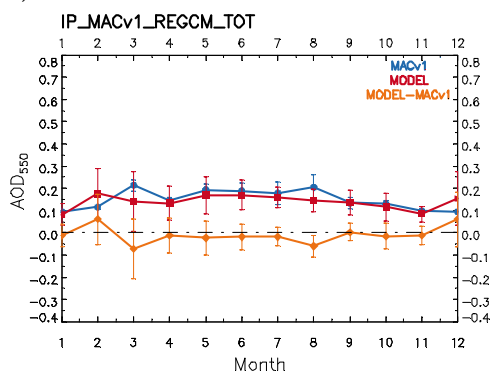

j)

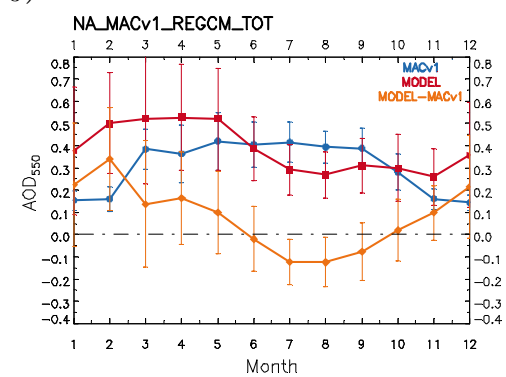

b)

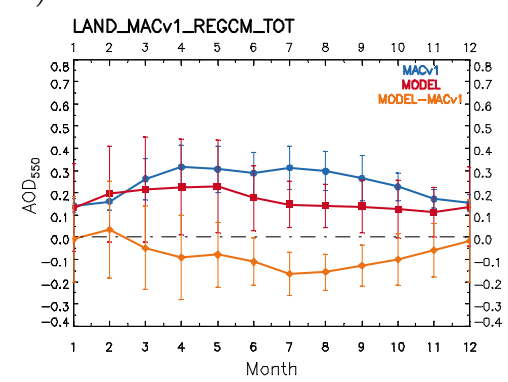

e)

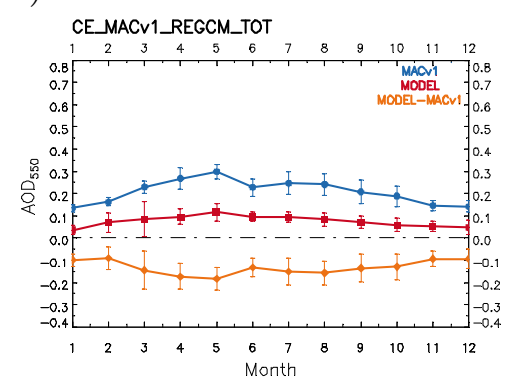

h)

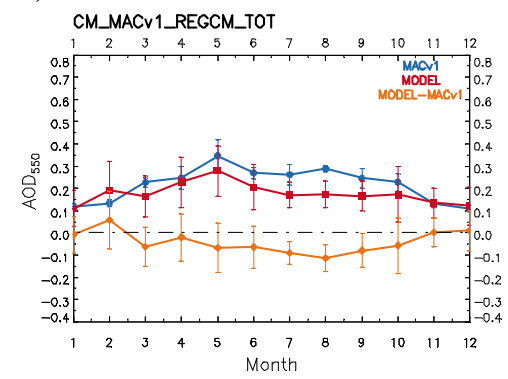

c)

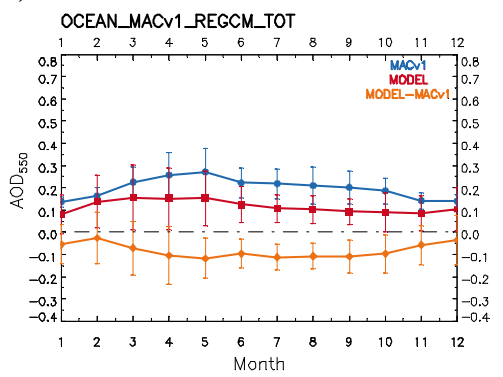

f)

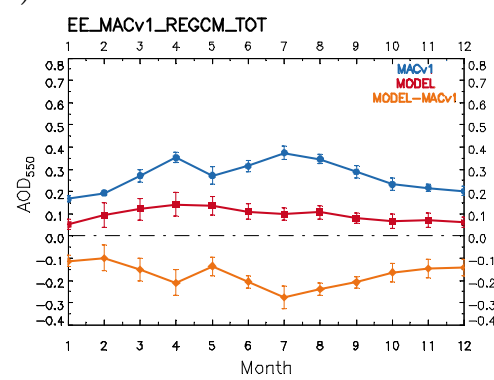

i)

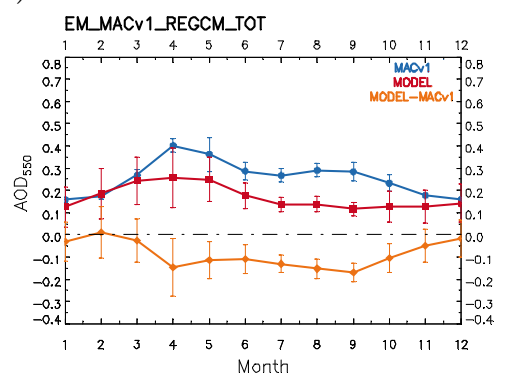

Figure S12. Seasonal variability of RegCM4 $\mathrm{AOD}_{550}$ (red), CM SAF AOD $\mathrm{AO}_{550}$ (blue) and their difference (orange) over (a) the whole Europe, (b) Land, (c) Ocean, (d) NE, (e) CE, (f) EE, (g) IP, (h) CM, (i) EM, (j) NA. 
Table S9. The same as Table S3 but for RegCM4 and MACv1 AOD $\mathrm{A}_{550}$.

\begin{tabular}{|c|c|c|c|c|c|c|c|c|c|c|c|c|c|c|c|}
\hline & \multicolumn{3}{|c|}{ ANN } & \multicolumn{3}{|c|}{ DJF } & \multicolumn{3}{|c|}{ MAM } & \multicolumn{3}{|c|}{ JJA } & \multicolumn{3}{|c|}{ SON } \\
\hline & MOD & SAT & bias & MOD & SAT & bias & MOD & SAT & bias & MOD & SAT & bias & MOD & SAT & bias \\
\hline $\mathrm{CE}$ & $0.08 \pm 0.04$ & $0.21 \pm 0.06$ & -63.59 & $0.05 \pm 0.04$ & $0.15 \pm 0.02$ & -64.92 & $0.1 \pm 0.06$ & $0.27 \pm 0.05$ & -62.94 & $0.09 \pm 0.03$ & $0.24 \pm 0.05$ & -61.58 & $0.06 \pm 0.03$ & $0.18 \pm 0.05$ & $\begin{array}{l}-66.18 \\
\end{array}$ \\
\hline $\mathrm{CM}$ & $0.18 \pm 0.1$ & $0.22 \pm 0.08$ & -19.33 & $0.14 \pm 0.11$ & $0.12 \pm 0.02$ & 16.18 & $0.22 \pm 0.12$ & $0.27 \pm 0.07$ & -18.45 & $0.18 \pm 0.08$ & $0.27 \pm 0.03$ & -32.93 & $0.16 \pm 0.09$ & $0.2 \pm 0.06$ & -22.36 \\
\hline $\mathrm{EE}$ & $0.1 \pm 0.05$ & $0.27 \pm 0.07$ & -64.58 & $0.07 \pm 0.04$ & $0.19 \pm 0.02$ & -62.44 & $0.13 \pm 0.05$ & $0.3 \pm 0.05$ & -55.47 & $0.1 \pm 0.03$ & $0.34 \pm 0.04$ & -69.58 & $0.07 \pm 0.03$ & $0.25 \pm 0.04$ & -70.17 \\
\hline EM & $0.17 \pm 0.1$ & $0.26 \pm 0.08$ & -34.19 & $0.15 \pm 0.1$ & $0.16 \pm 0.02$ & -7.73 & $0.25 \pm 0.11$ & $0.34 \pm 0.07$ & -27.7 & $0.15 \pm 0.05$ & $0.28 \pm 0.03$ & -46.68 & $0.12 \pm 0.06$ & $0.23 \pm 0.06$ & -46.39 \\
\hline $\mathrm{EU}$ & $0.15 \pm 0.15$ & $0.23 \pm 0.1$ & -35.27 & $0.14 \pm 0.17$ & $0.15 \pm 0.04$ & -7.91 & $0.20 \pm 0.20$ & $0.28 \pm 0.1$ & -29.36 & $0.14 \pm 0.11$ & $0.27 \pm 0.1$ & -48.38 & $0.11 \pm 0.11$ & $0.2 \pm 0.08$ & -45.12 \\
\hline IP & $0.14 \pm 0.09$ & $0.15 \pm 0.05$ & -7.43 & $0.14 \pm 0.11$ & $0.1 \pm 0.01$ & 33.79 & $0.15 \pm 0.1$ & $0.18 \pm 0.04$ & -19.27 & $0.16 \pm 0.06$ & $0.19 \pm 0.05$ & -17.09 & $0.11 \pm 0.06$ & $0.12 \pm 0.02$ & -7.11 \\
\hline LA & $0.17 \pm 0.17$ & $0.24 \pm 0.1$ & -32.09 & $0.16 \pm 0.2$ & $0.15 \pm 0.04$ & 2.66 & $0.22 \pm 0.22$ & $0.29 \pm 0.1$ & -24.56 & $0.15 \pm 0.12$ & $0.3 \pm 0.1$ & -48.22 & $0.13 \pm 0.12$ & $0.22 \pm 0.08$ & -42.97 \\
\hline NA & $0.39 \pm 0.22$ & $0.31 \pm 0.14$ & 25.01 & $0.41 \pm 0.26$ & $0.15 \pm 0.05$ & 171.38 & $0.52 \pm 0.26$ & $0.39 \pm 0.12$ & 34.17 & $0.32 \pm 0.13$ & $0.4 \pm 0.09$ & -22.08 & $0.29 \pm 0.14$ & $0.28 \pm 0.12$ & 4.62 \\
\hline $\mathrm{NE}$ & $0.05 \pm 0.03$ & $0.2 \pm 0.06$ & -75.92 & $0.03 \pm 0.02$ & $0.15 \pm 0.02$ & -76.94 & $0.07 \pm 0.04$ & $0.22 \pm 0.07$ & -70.23 & $0.06 \pm 0.02$ & $0.22 \pm 0.05$ & -74.61 & $0.03 \pm 0.02$ & $0.2 \pm 0.04$ & -82.95 \\
\hline $\mathrm{OC}$ & $0.12 \pm 0.1$ & $0.2 \pm 0.08$ & -41.95 & $0.11 \pm 0.11$ & $0.15 \pm 0.03$ & -26.75 & $0.15 \pm 0.14$ & $0.25 \pm 0.09$ & -39 & $0.11 \pm 0.07$ & $0.22 \pm 0.07$ & -48.76 & $0.09 \pm 0.08$ & $0.18 \pm 0.06$ & -49.72 \\
\hline
\end{tabular}


Table S10. Average RegCM4 AOD 550 , MACv1 AOD 550 , their difference, the corresponding NMB and the statistical significance of the results at the $95 \%$ confidence level due to a two independent sample t-test per season and region. The NSD, MNMB and the RMSE are also given along with Pearson's correlation coefficient (R), the number of observations used and the coefficients $\mathrm{a}$ and $\mathrm{b}$ of the regression line (RegCM4=a-MACv1+b). The region are listed in alphabetic order.

\begin{tabular}{|c|c|c|c|c|c|c|c|c|c|c|c|c|c|}
\hline Season & Region & REG & MACv1 & Diff. & NMB & Signif. & $\mathrm{R}$ & Obs. & $\mathrm{b}$ & $\mathrm{a}$ & NSD & MNMB & RMSE \\
\hline DJF & $\mathrm{CE}$ & 0.05 & 0.15 & -0.09 & -64.92 & 1 & 0.07 & 13209 & 0.04 & 0.10 & 1.55 & -102.94 & 0.10 \\
\hline JJA & $\mathrm{CE}$ & 0.09 & 0.24 & -0.15 & -61.58 & 1 & 0.00 & 13986 & 0.09 & 0.00 & 0.54 & -88.55 & 0.16 \\
\hline MAM & $\mathrm{CE}$ & 0.10 & 0.27 & -0.17 & -62.94 & 1 & 0.11 & 13986 & 0.06 & 0.13 & 1.21 & -97.02 & 0.18 \\
\hline SON & $\mathrm{CE}$ & 0.06 & 0.18 & -0.12 & -66.18 & 1 & 0.05 & 13986 & 0.06 & 0.03 & 0.57 & -99.14 & 0.13 \\
\hline TOT & $\mathrm{CE}$ & 0.08 & 0.21 & -0.13 & -63.59 & 1 & 0.37 & 55167 & 0.02 & 0.25 & 0.68 & -96.83 & 0.15 \\
\hline DJF & $\mathrm{CM}$ & 0.14 & 0.12 & 0.02 & 16.18 & 1 & 0.12 & 7803 & 0.05 & 0.73 & 6.02 & -10.77 & 0.11 \\
\hline JJA & $\mathrm{CM}$ & 0.18 & 0.27 & -0.09 & -32.93 & 1 & 0.34 & 8262 & -0.04 & 0.82 & 2.41 & -44.66 & 0.12 \\
\hline MAM & $\mathrm{CM}$ & 0.22 & 0.27 & -0.05 & -18.45 & 1 & 0.47 & 8262 & 0.02 & 0.75 & 1.59 & -29.27 & 0.12 \\
\hline $\mathrm{SON}$ & $\mathrm{CM}$ & 0.16 & 0.20 & -0.05 & -22.36 & 1 & 0.22 & 8262 & 0.09 & 0.33 & 1.52 & -31.87 & 0.11 \\
\hline TOT & $\mathrm{CM}$ & 0.18 & 0.22 & -0.04 & -19.33 & 1 & 0.38 & 32589 & 0.07 & 0.50 & 1.30 & -29.40 & 0.11 \\
\hline DJF & $\mathrm{EE}$ & 0.07 & 0.19 & -0.12 & -62.44 & 1 & 0.10 & 14637 & 0.03 & 0.21 & 1.97 & -96.30 & 0.12 \\
\hline JJA & $\mathrm{EE}$ & 0.10 & 0.34 & -0.24 & -69.58 & 1 & 0.00 & 15498 & 0.11 & 0.00 & 0.87 & -107.59 & 0.24 \\
\hline MAM & $\mathrm{EE}$ & 0.13 & 0.30 & -0.17 & -55.47 & 1 & 0.25 & 15498 & 0.06 & 0.26 & 1.01 & -79.22 & 0.18 \\
\hline $\mathrm{SON}$ & $\mathrm{EE}$ & 0.07 & 0.25 & -0.17 & -70.17 & 1 & 0.16 & 15498 & 0.04 & 0.12 & 0.74 & -109.66 & 0.18 \\
\hline TOT & $\mathrm{EE}$ & 0.10 & 0.27 & -0.17 & -64.58 & 1 & 0.41 & 61131 & 0.02 & 0.27 & 0.67 & -98.22 & 0.19 \\
\hline DJF & EM & 0.15 & 0.16 & -0.01 & -7.73 & 1 & 0.19 & 18819 & -0.04 & 1.17 & 6.14 & -25.40 & 0.10 \\
\hline JJA & EM & 0.15 & 0.28 & -0.13 & -46.68 & 1 & 0.19 & 19926 & 0.08 & 0.27 & 1.40 & -63.17 & 0.14 \\
\hline MAM & EM & 0.25 & 0.34 & -0.10 & -27.70 & 1 & 0.31 & 19926 & 0.09 & 0.47 & 1.53 & -37.28 & 0.15 \\
\hline SON & EM & 0.12 & 0.23 & -0.11 & -46.39 & 1 & 0.07 & 19926 & 0.10 & 0.08 & 1.12 & -64.82 & 0.13 \\
\hline TOT & $\mathrm{EM}$ & 0.17 & 0.26 & -0.09 & -34.19 & 1 & 0.40 & 78597 & 0.05 & 0.47 & 1.18 & -47.98 & 0.13 \\
\hline DJF & $\mathrm{EU}$ & 0.14 & 0.15 & -0.01 & -7.91 & 1 & 0.11 & 127942 & 0.06 & 0.52 & 4.92 & -50.42 & 0.17 \\
\hline JJA & $\mathrm{EU}$ & 0.14 & 0.27 & -0.13 & -48.38 & 1 & 0.62 & 135468 & -0.04 & 0.68 & 1.10 & -74.45 & 0.16 \\
\hline MAM & $\mathrm{EU}$ & 0.20 & 0.28 & -0.08 & -29.36 & 1 & 0.60 & 135468 & -0.13 & 1.18 & 1.95 & -60.14 & 0.18 \\
\hline SON & $\mathrm{EU}$ & 0.11 & 0.20 & -0.09 & -45.12 & 1 & 0.42 & 135468 & -0.01 & 0.57 & 1.37 & -75.24 & 0.14 \\
\hline TOT & $\mathrm{EU}$ & 0.15 & 0.23 & -0.08 & -35.27 & 1 & 0.44 & 534346 & -0.01 & 0.70 & 1.59 & -65.27 & 0.16 \\
\hline DJF & IP & 0.14 & 0.10 & 0.03 & 33.79 & 1 & 0.12 & 6069 & 0.04 & 0.97 & 8.30 & 2.17 & 0.11 \\
\hline JJA & IP & 0.16 & 0.19 & -0.03 & -17.09 & 1 & 0.49 & 6426 & 0.05 & 0.57 & 1.16 & -21.32 & 0.06 \\
\hline MAM & IP & 0.15 & 0.18 & -0.04 & -19.27 & 1 & 0.17 & 6426 & 0.06 & 0.47 & 2.84 & -36.02 & 0.11 \\
\hline SON & IP & 0.11 & 0.12 & -0.01 & -7.11 & 1 & 0.56 & 6426 & -0.04 & 1.28 & 2.28 & -15.87 & 0.05 \\
\hline TOT & IP & 0.14 & 0.15 & -0.01 & -7.43 & 1 & 0.29 & 25347 & 0.07 & 0.48 & 1.67 & -18.04 & 0.09 \\
\hline DJF & LA & 0.16 & 0.15 & 0.00 & 2.66 & 1 & 0.09 & 80767 & 0.09 & 0.47 & 5.42 & -45.15 & 0.20 \\
\hline JJA & LA & 0.15 & 0.30 & -0.14 & -48.22 & 1 & 0.59 & 85518 & -0.07 & 0.74 & 1.25 & -75.21 & 0.17 \\
\hline MAM & LA & 0.22 & 0.29 & -0.07 & -24.56 & 1 & 0.62 & 85518 & -0.18 & 1.36 & 2.19 & -54.69 & 0.19 \\
\hline $\mathrm{SON}$ & LA & 0.13 & 0.22 & -0.10 & -42.97 & 1 & 0.43 & 85518 & -0.01 & 0.63 & 1.47 & -72.91 & 0.15 \\
\hline TOT & LA & 0.17 & 0.24 & -0.08 & -32.09 & 1 & 0.42 & 337321 & -0.01 & 0.71 & 1.71 & -62.23 & 0.18 \\
\hline DJF & NA & 0.41 & 0.15 & 0.26 & 171.38 & 1 & 0.18 & 18360 & 0.26 & 1.02 & 5.62 & 74.32 & 0.37 \\
\hline JJA & NA & 0.32 & 0.40 & -0.09 & -22.08 & 1 & 0.35 & 19440 & 0.10 & 0.52 & 1.48 & -29.75 & 0.16 \\
\hline MAM & NA & 0.52 & 0.39 & 0.13 & 34.17 & 1 & 0.43 & 19440 & 0.17 & 0.90 & 2.12 & 21.60 & 0.27 \\
\hline SON & NA & 0.29 & 0.28 & 0.01 & 4.62 & 1 & 0.32 & 19440 & 0.19 & 0.37 & 1.13 & 4.05 & 0.15 \\
\hline TOT & NA & 0.39 & 0.31 & 0.08 & 25.01 & 1 & 0.22 & 76680 & 0.28 & 0.35 & 1.59 & 16.76 & 0.25 \\
\hline DJF & $\mathrm{NE}$ & 0.03 & 0.15 & -0.11 & -76.94 & 1 & 0.17 & 15691 & 0.02 & 0.12 & 0.68 & -126.86 & 0.11 \\
\hline JJA & $\mathrm{NE}$ & 0.06 & 0.22 & -0.17 & -74.61 & 1 & 0.16 & 16614 & 0.04 & 0.05 & 0.33 & -118.24 & 0.17 \\
\hline MAM & $\mathrm{NE}$ & 0.07 & 0.22 & -0.16 & -70.23 & 1 & 0.16 & 16614 & 0.04 & 0.10 & 0.60 & -110.20 & 0.17 \\
\hline SON & $\mathrm{NE}$ & 0.03 & 0.20 & -0.17 & -82.95 & 1 & -0.09 & 16614 & 0.04 & -0.04 & 0.48 & -141.96 & 0.17 \\
\hline TOT & $\mathrm{NE}$ & 0.05 & 0.20 & -0.15 & -75.92 & 1 & 0.29 & 65533 & 0.02 & 0.15 & 0.50 & -124.28 & 0.16 \\
\hline DJF & $\mathrm{OC}$ & 0.11 & 0.15 & -0.04 & -26.75 & 1 & 0.14 & 47175 & 0.04 & 0.46 & 3.33 & -59.45 & 0.11 \\
\hline JJA & $\mathrm{OC}$ & 0.11 & 0.22 & -0.11 & -48.76 & 1 & 0.65 & 49950 & -0.02 & 0.62 & 0.96 & -73.14 & 0.12 \\
\hline MAM & $\mathrm{OC}$ & 0.15 & 0.25 & -0.10 & -39.00 & 1 & 0.54 & 49950 & -0.04 & 0.77 & 1.44 & -69.48 & 0.15 \\
\hline SON & $\mathrm{OC}$ & 0.09 & 0.18 & -0.09 & -49.72 & 1 & 0.27 & 49950 & 0.03 & 0.32 & 1.20 & -79.25 & 0.12 \\
\hline TOT & $\mathrm{OC}$ & 0.12 & 0.20 & -0.08 & -41.95 & 1 & 0.45 & 197025 & 0.00 & 0.58 & 1.29 & -70.48 & 0.13 \\
\hline
\end{tabular}




\section{Aerosol Asymmetry Factor}

a)

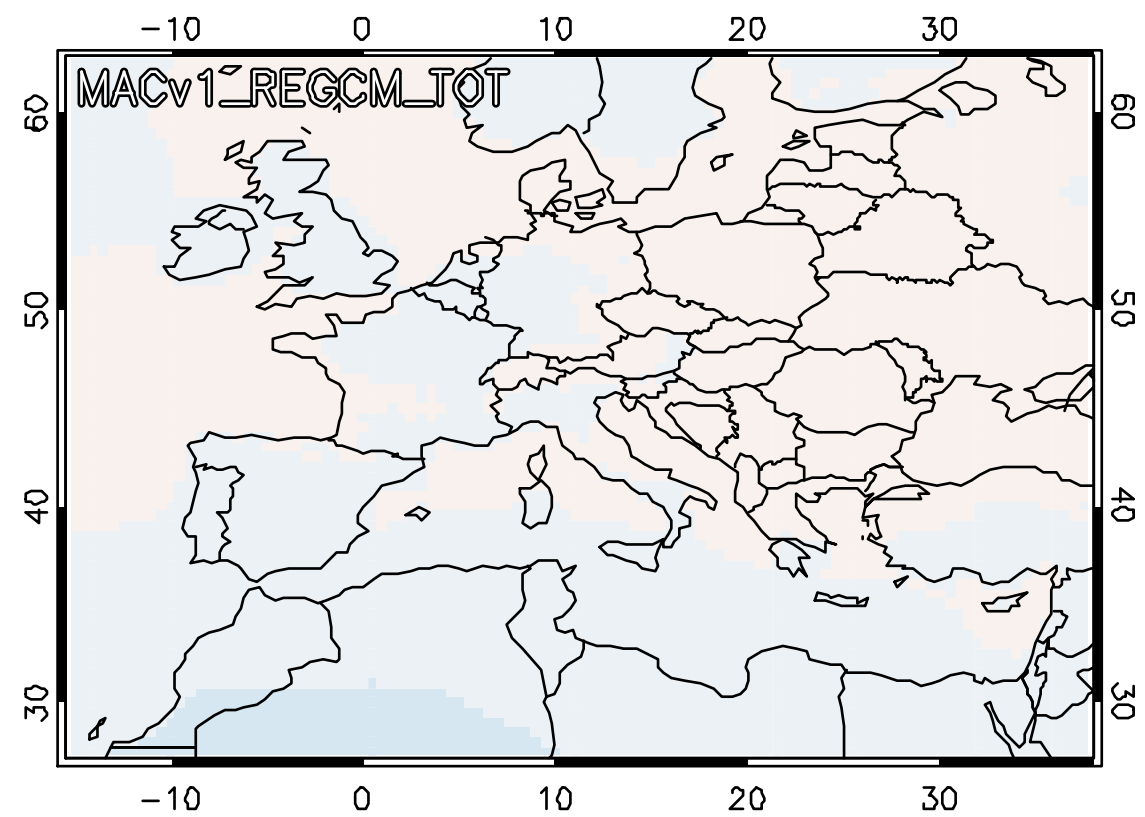

b)

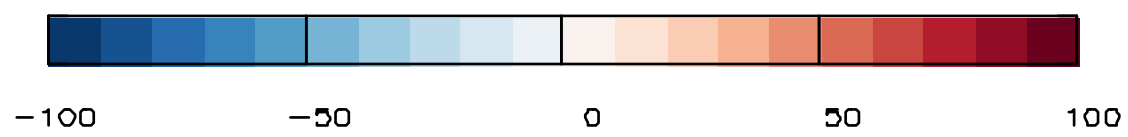

EUROPE_MACV1_REGCM_TOT

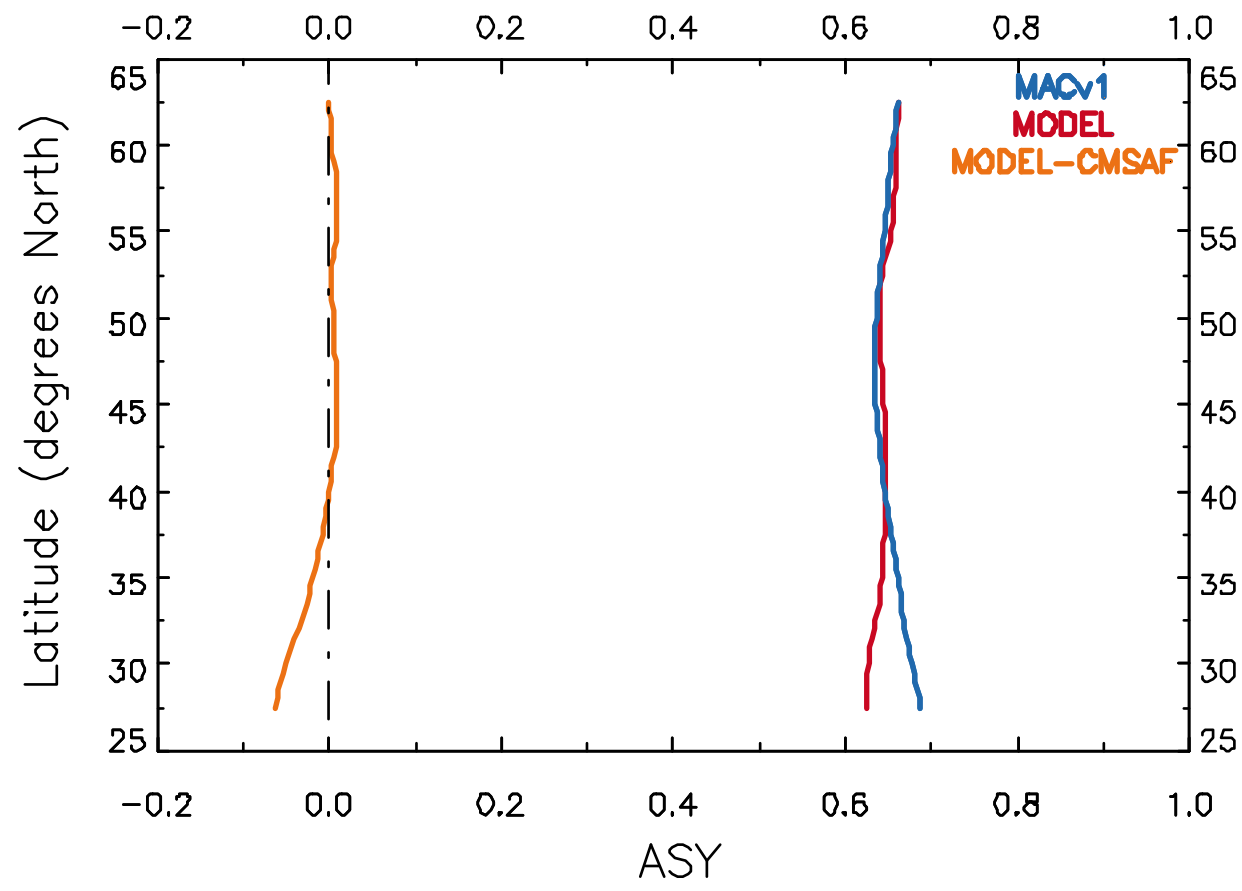

Figure S13. (a) NMB patterns of RegCM4-MACv1 ASY and (b) Latitudinal variability of RegCM4 ASY (red), MACv1 ASY (blue) and their difference (orange) over Europe. 
a)

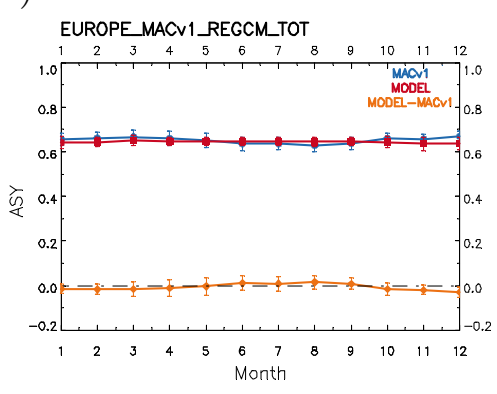

d)

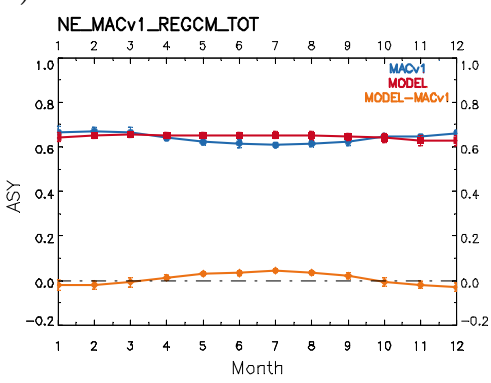

g)

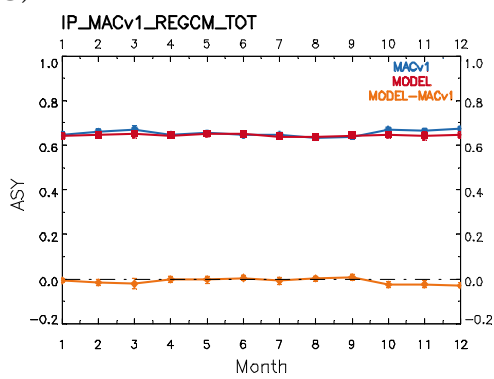

j)

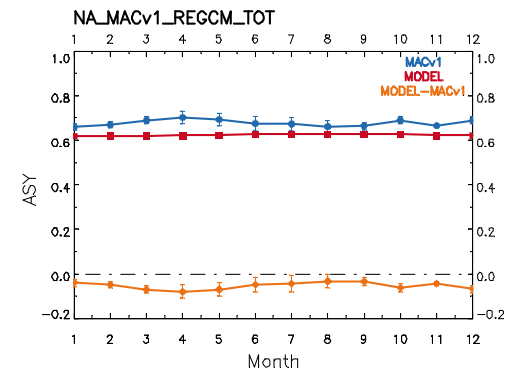

b)

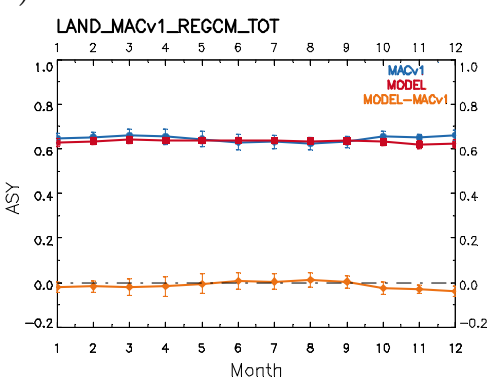

e)

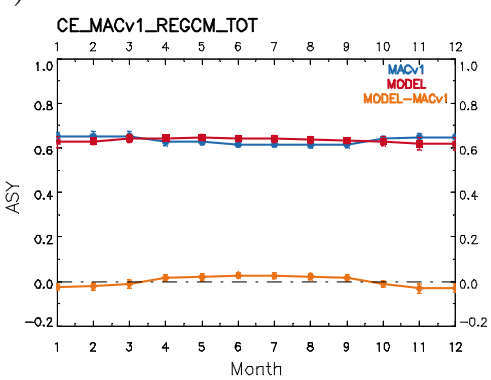

h)

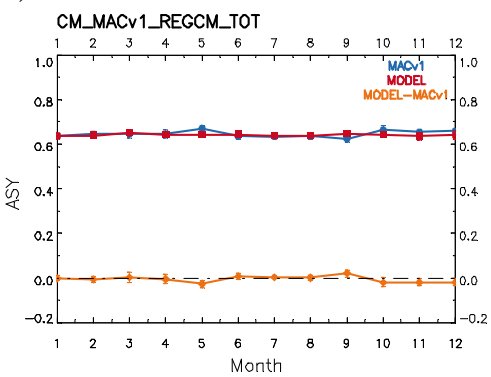

c)

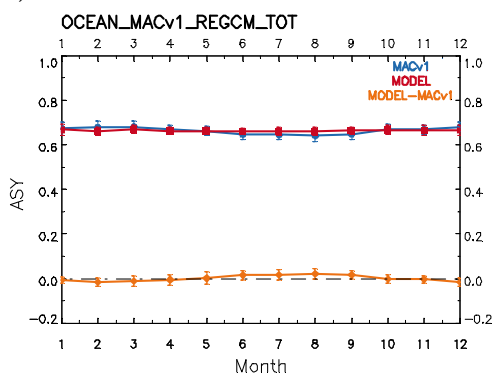

f)

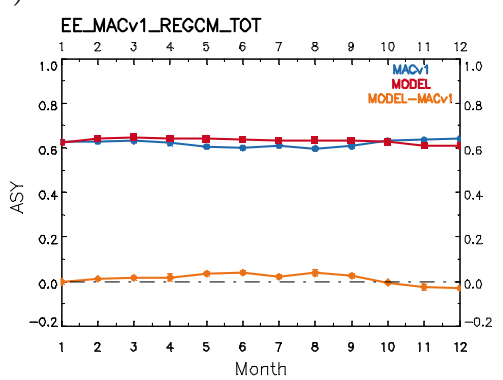

i)

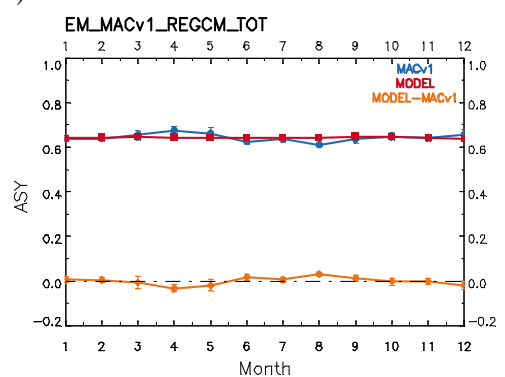

Figure S14. Seasonal variability of RegCM4 ASY (red), MACv1 ASY (blue) and their difference (orange) over (a) the whole Europe, (b) Land, (c) Ocean, (d) NE, (e) CE, (f) EE, (g) IP, (h) CM, (i) EM, (j) NA. 
Table S11. Average RegCM4 ASY, MACv1 ASY, their difference, the corresponding NMB and the statistical significance of the results at the $95 \%$ confidence level due to a two independent sample t-test for each region of interest. The NSD, MNMB and the RMSE are also given along with Pearson's correlation coefficient (R), the number of observations used and the coefficients $\mathrm{a}$ and $\mathrm{b}$ of the regression line $(\mathrm{RegCM} 4=\mathrm{a} \cdot \mathrm{MACv} 1+\mathrm{b})$. The region are listed in alphabetic order.

\begin{tabular}{|c|c|c|c|c|c|c|c|c|c|c|c|c|c|}
\hline Season & Region & REG & MACv1 & Diff. & NMB & Signif. & $\mathrm{R}$ & Obs. & $\mathrm{b}$ & $\mathrm{a}$ & NSD & MNMB & RMSE \\
\hline TOT & $\mathrm{CE}$ & 0.63 & 0.63 & 0.00 & 0.03 & 0 & 0.23 & 36519 & 0.50 & 0.22 & 0.96 & 0.04 & 0.03 \\
\hline TOT & $\mathrm{CM}$ & 0.64 & 0.65 & 0.00 & -0.74 & 1 & 0.11 & 21573 & 0.59 & 0.07 & 0.66 & -0.72 & 0.02 \\
\hline TOT & $\mathrm{EE}$ & 0.63 & 0.62 & 0.01 & 2.08 & 1 & -0.23 & 40467 & 0.76 & -0.21 & 0.92 & 2.06 & 0.03 \\
\hline TOT & EM & 0.64 & 0.64 & 0.00 & -0.04 & 1 & 0.07 & 52029 & 0.62 & 0.03 & 0.41 & 0.01 & 0.02 \\
\hline TOT & $\mathrm{EU}$ & 0.64 & 0.65 & -0.01 & -1.07 & 1 & 0.30 & 353722 & 0.50 & 0.22 & 0.74 & -1.02 & 0.03 \\
\hline TOT & IP & 0.64 & 0.65 & -0.01 & -1.52 & 1 & 0.30 & 16779 & 0.48 & 0.25 & 0.83 & -1.52 & 0.02 \\
\hline TOT & NA & 0.62 & 0.68 & -0.05 & -7.87 & 1 & -0.16 & 50760 & 0.66 & -0.06 & 0.36 & -8.13 & 0.06 \\
\hline TOT & LA & 0.63 & 0.64 & -0.01 & -1.79 & 1 & -0.09 & 223297 & 0.66 & -0.05 & 0.52 & -1.72 & 0.04 \\
\hline TOT & $\mathrm{NE}$ & 0.65 & 0.64 & 0.01 & 1.03 & 1 & 0.21 & 43381 & 0.55 & 0.15 & 0.71 & 1.07 & 0.03 \\
\hline TOT & $\mathrm{OC}$ & 0.66 & 0.66 & 0.00 & 0.13 & 1 & 0.53 & 130425 & 0.42 & 0.36 & 0.69 & 0.17 & 0.02 \\
\hline
\end{tabular}




\section{Aerosol Single Scattering Albedo}

a)

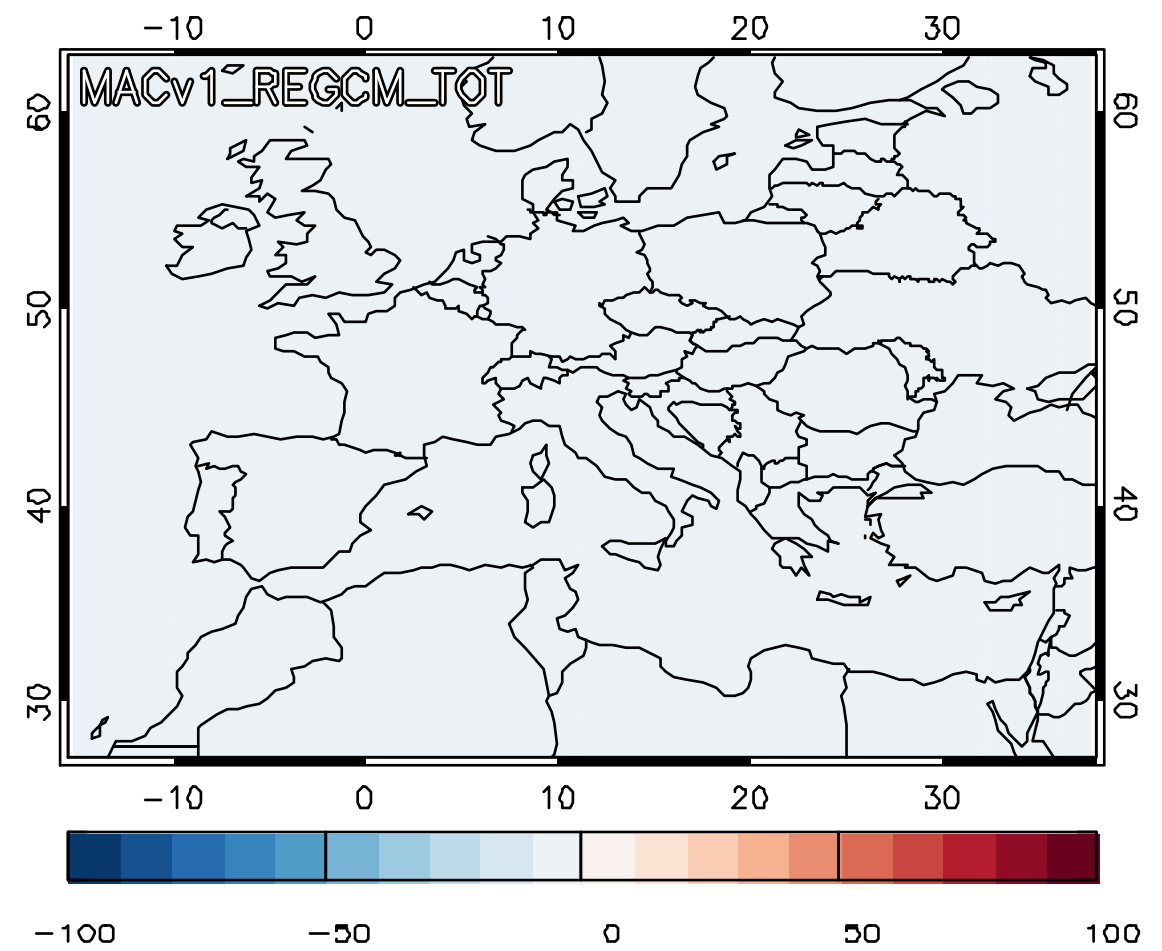

b)

\section{EUROPE_MACV1_REGCM_TOT}

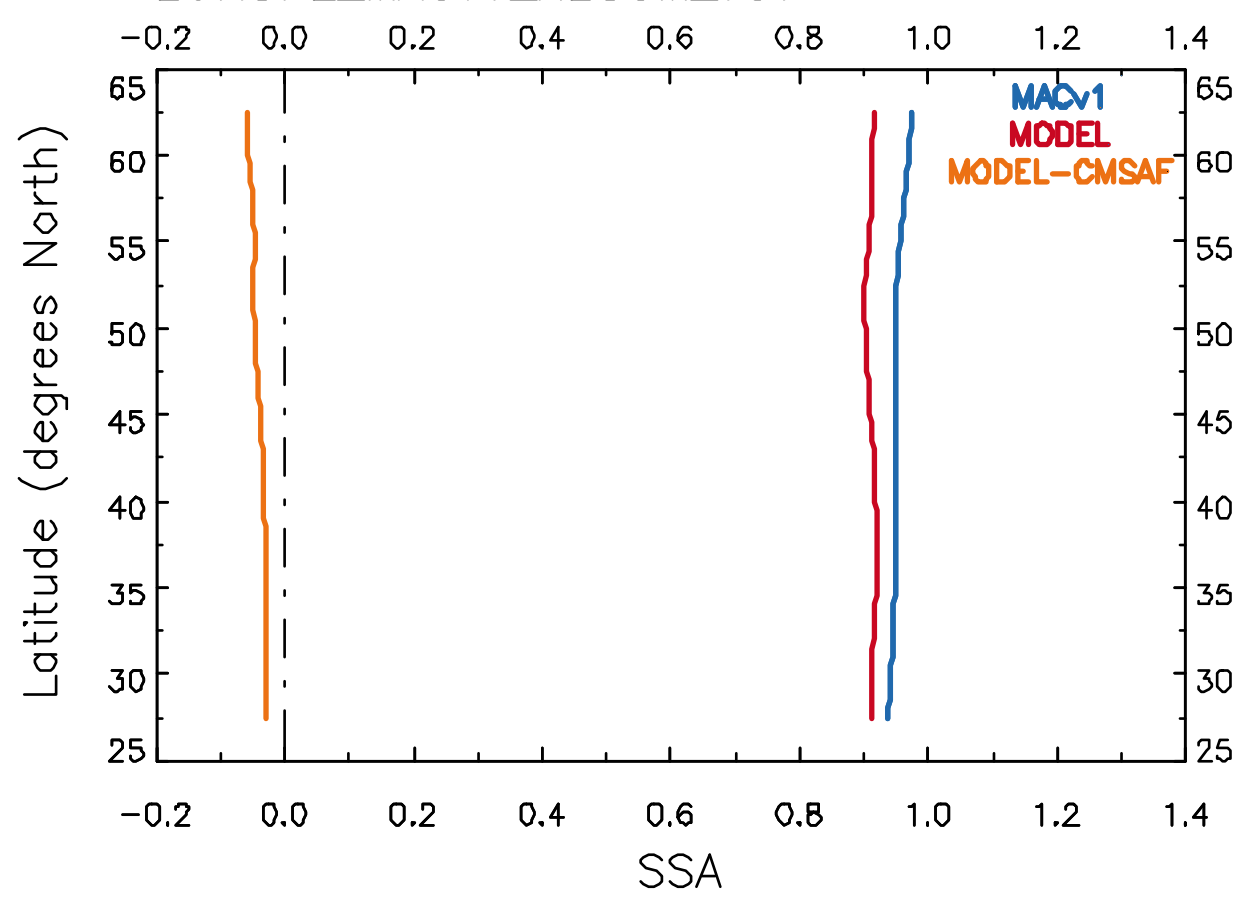

Figure S15. (a) NMB patterns of RegCM4-MACv1 SSA and (b) Latitudinal variability of RegCM4 SSA (red), MACv1 SSA (blue) and their difference (orange) over Europe. 
a)

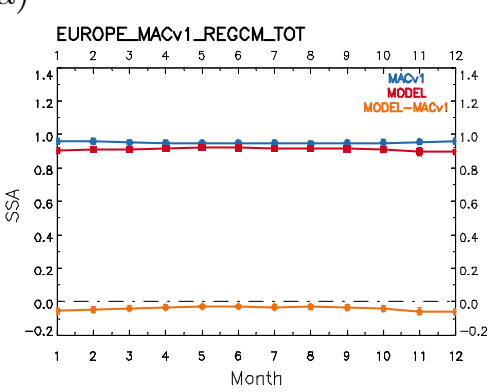

d)

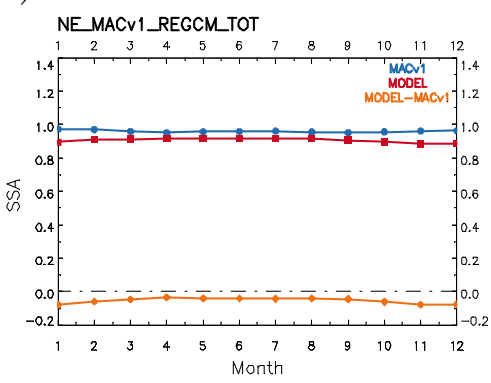

g)

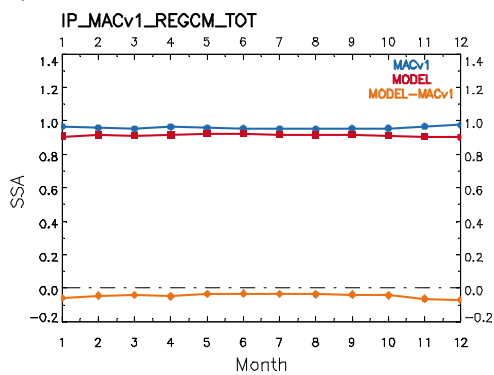

j)

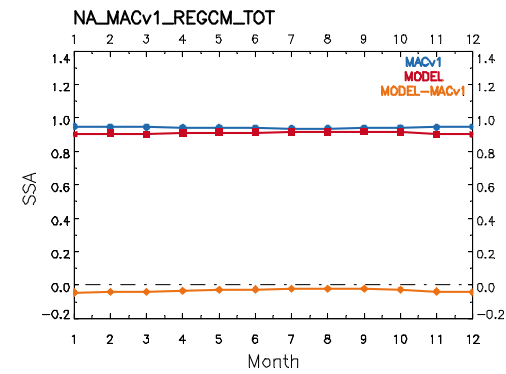

b)

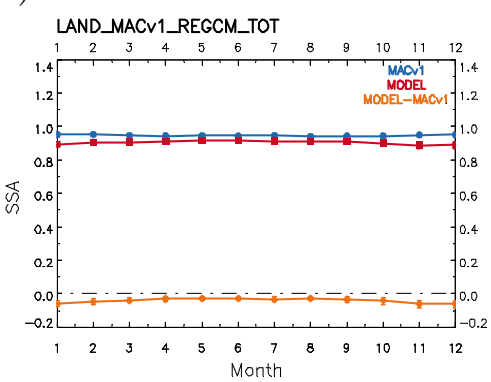

e)

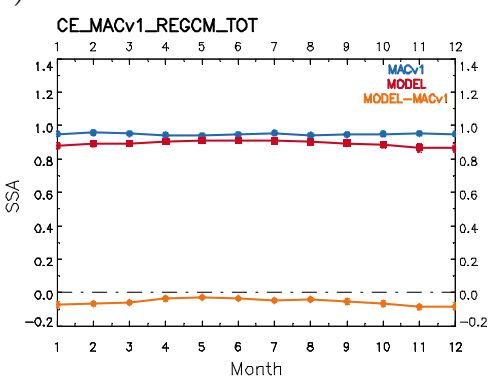

h)

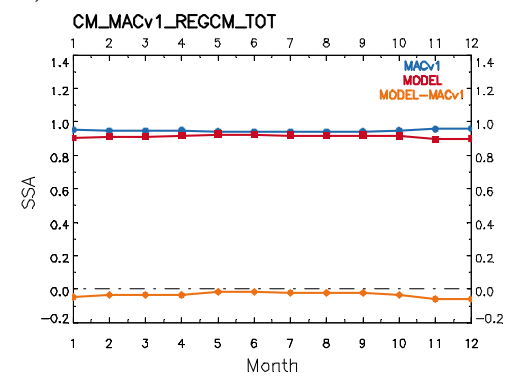

c)

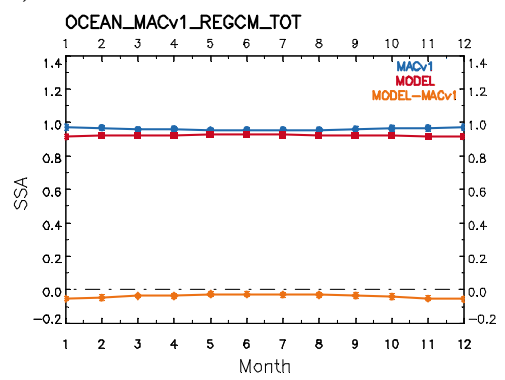

f)

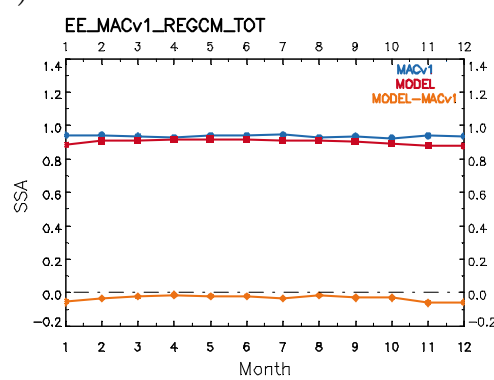

i)

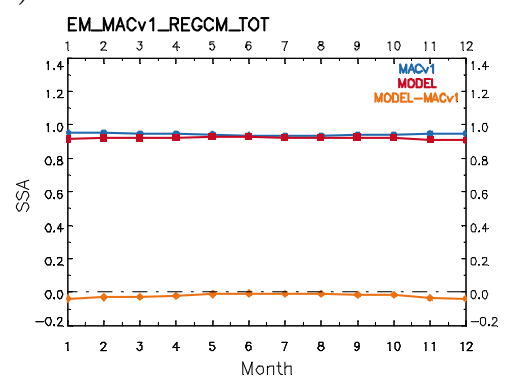

Figure S16. Seasonal variability of RegCM4 SSA (red), MACv1 SSA (blue) and their difference (orange) over (a) the whole Europe, (b) Land, (c) Ocean, (d) NE, (e) CE, (f) EE, (g) IP, (h) CM, (i) EM, (j) NA. 
Table S12. Average RegCM4 SSA, MACv1 SSA, their difference, the corresponding NMB and the statistical significance of the results at the $95 \%$ confidence level due to a two independent sample t-test for each region of interest. The NSD, MNMB and the RMSE are also given along with Pearson's correlation coefficient (R), the number of observations used and the coefficients $\mathrm{a}$ and $\mathrm{b}$ of the regression line $(\mathrm{RegCM} 4=\mathrm{a} \cdot \mathrm{MACv} 1+\mathrm{b})$. The region are listed in alphabetic order.

\begin{tabular}{|c|c|c|c|c|c|c|c|c|c|c|c|c|c|}
\hline Season & Region & REG & MACv1 & Diff. & NMB & Signif. & $\mathrm{R}$ & Obs. & $\mathrm{b}$ & $\mathrm{a}$ & NSD & MNMB & RMSE \\
\hline TOT & $\mathrm{CE}$ & 0.89 & 0.95 & -0.06 & -5.88 & 1 & 0.26 & 36519 & 0.54 & 0.37 & 1.46 & -6.07 & 0.06 \\
\hline TOT & $\mathrm{CM}$ & 0.91 & 0.95 & -0.03 & -3.53 & 1 & -0.30 & 21573 & 1.37 & -0.49 & 1.61 & -3.60 & 0.04 \\
\hline TOT & $\mathrm{EE}$ & 0.90 & 0.94 & -0.03 & -3.54 & 1 & 0.10 & 40467 & 0.72 & 0.20 & 2.03 & -3.62 & 0.04 \\
\hline TOT & EM & 0.92 & 0.94 & -0.02 & -2.32 & 1 & -0.19 & 52029 & 1.11 & -0.20 & 1.06 & -2.34 & 0.03 \\
\hline TOT & $\mathrm{EU}$ & 0.91 & 0.95 & -0.04 & -4.23 & 1 & 0.29 & 353722 & 0.65 & 0.28 & 0.97 & -4.33 & 0.04 \\
\hline TOT & IP & 0.91 & 0.96 & -0.05 & -4.75 & 1 & -0.14 & 16779 & 1.05 & -0.14 & 1.05 & -4.87 & 0.05 \\
\hline TOT & NA & 0.91 & 0.94 & -0.03 & -3.46 & 1 & -0.23 & 50760 & 1.11 & -0.22 & 0.96 & -3.52 & 0.03 \\
\hline TOT & $\mathrm{NE}$ & 0.91 & 0.96 & -0.05 & -5.57 & 1 & 0.13 & 43381 & 0.75 & 0.16 & 1.25 & -5.73 & 0.06 \\
\hline TOT & LA & 0.90 & 0.95 & -0.04 & -4.33 & 1 & 0.03 & 223297 & 0.87 & 0.04 & 1.22 & -4.43 & 0.05 \\
\hline TOT & $\mathrm{OC}$ & 0.92 & 0.96 & -0.04 & -4.07 & 1 & 0.22 & 130425 & 0.81 & 0.12 & 0.53 & -4.14 & 0.04 \\
\hline
\end{tabular}




\section{Water Vapor}

a)

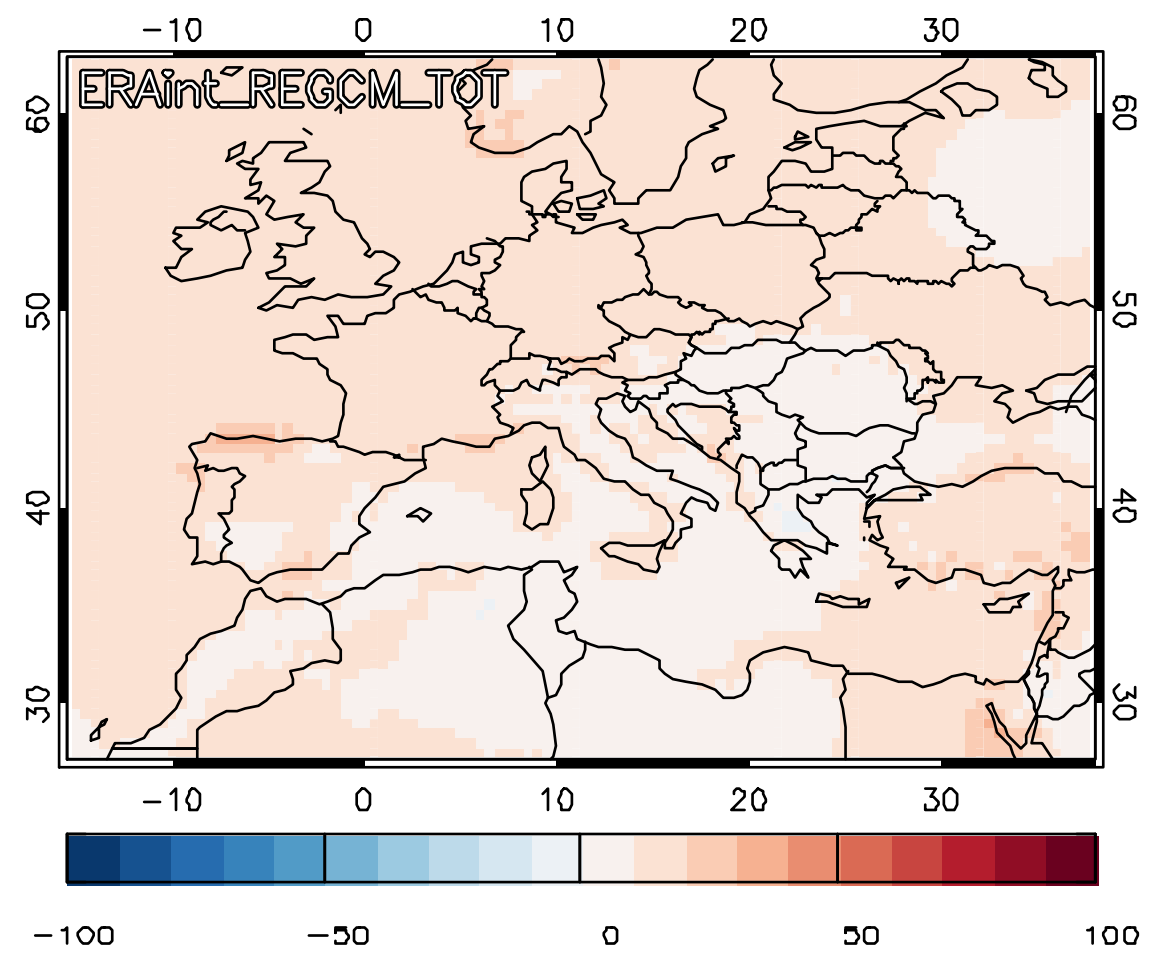

b)

\section{EUROPE ERAint_REGCM_TOT}

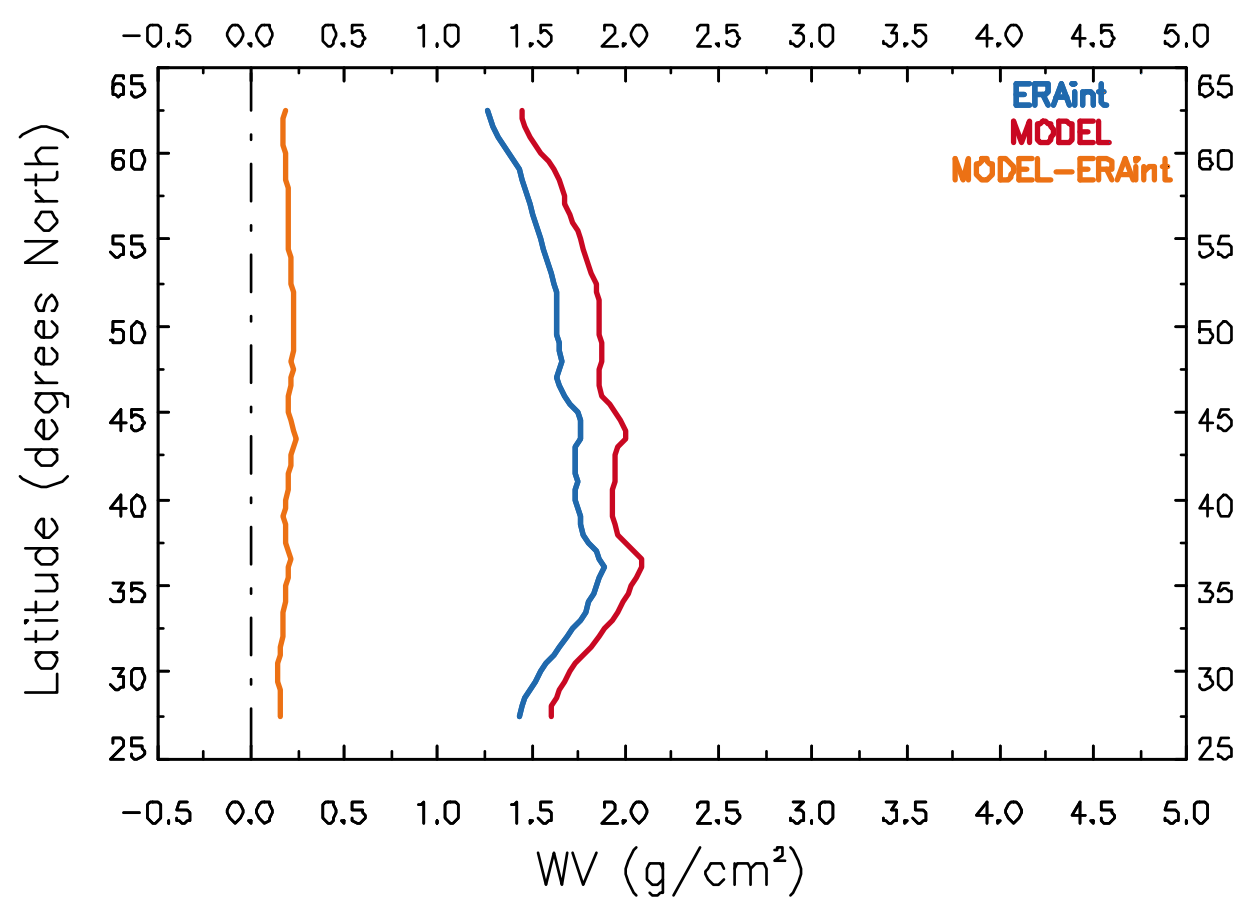

Figure S17. (a) NMB patterns of RegCM4-ERA-Interim WV and (b) Latitudinal variability of RegCM4 WV (red), ERA-Interim WV (blue) and their difference (orange) over Europe. 
a)

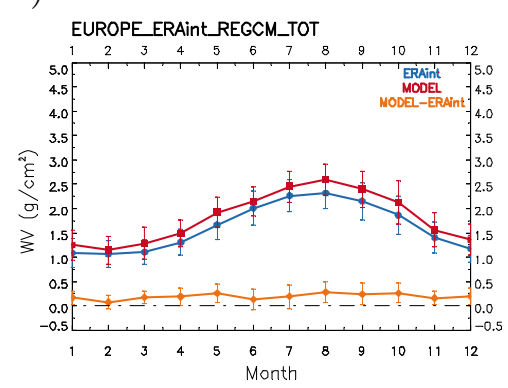

d)

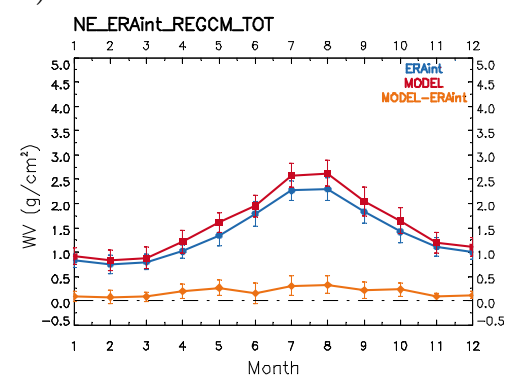

g)

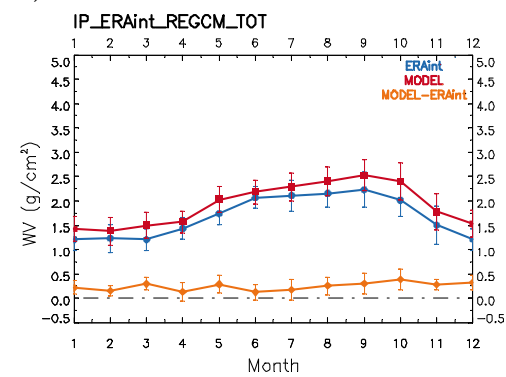

j)

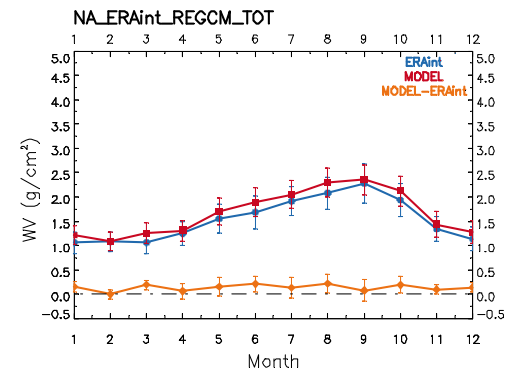

b)

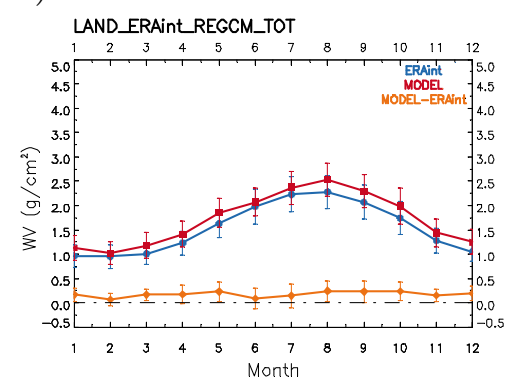

e)

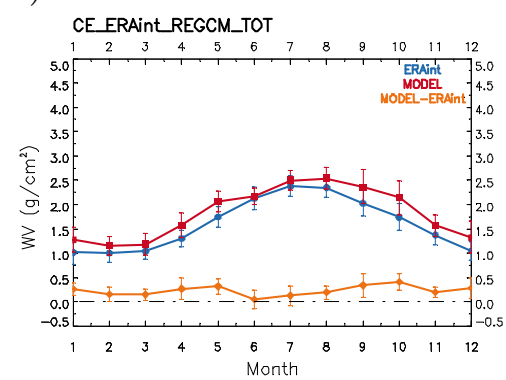

h)

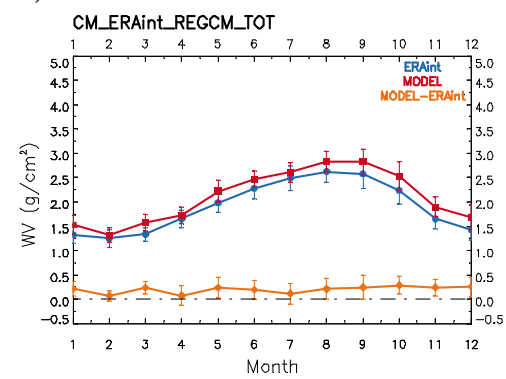

c)

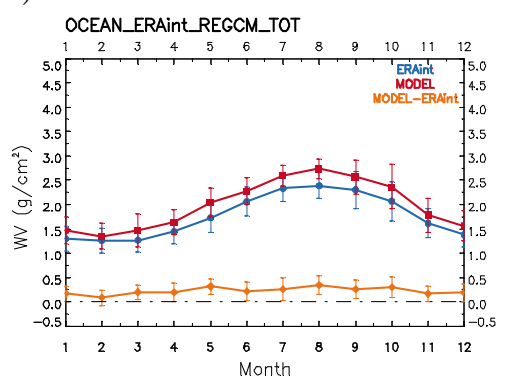

f)

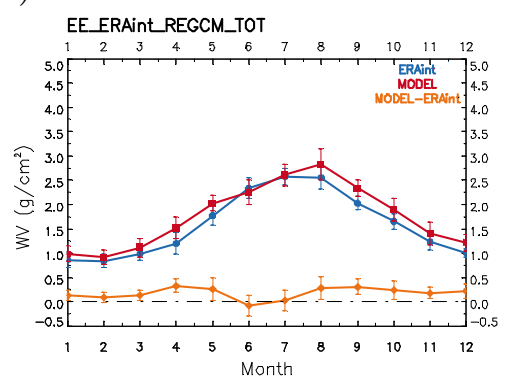

i)

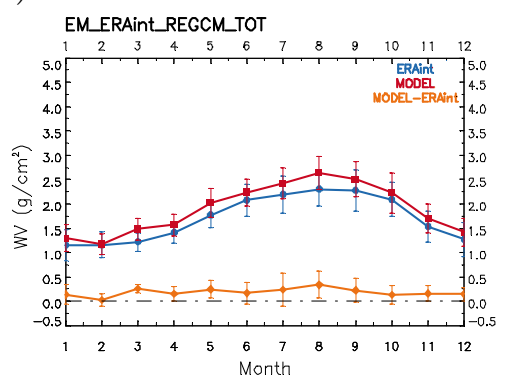

Figure S18. Seasonal variability of RegCM4 WV (red), ERA-Interim WV (blue) and their difference (orange) over (a) the whole Europe, (b) Land, (c) Ocean, (d) NE, (e) CE, (f) EE, (g) IP, (h) CM, (i) EM, (j) NA. 
Table S13. Average RegCM4 WV, ERA-Interim WV, their difference, the NMB and the statistical significance of the results at the $95 \%$ confidence level due to a two independent sample t-test for each region of interest. The NSD, MNMB and the RMSE are also given along with Pearson's correlation coefficient (R), the number of observations used and the coefficients $\mathrm{a}$ and $\mathrm{b}$ of the regression line $(\mathrm{RegCM} 4=\mathrm{a} \cdot \mathrm{ERAint}+\mathrm{b})$. The region are listed in alphabetic order.

\begin{tabular}{|c|c|c|c|c|c|c|c|c|c|c|c|c|c|}
\hline Season & Region & REG & ERAint & Diff. & NMB & Signif. & $\mathrm{R}$ & Obs. & $\mathrm{b}$ & $\mathrm{a}$ & NSD & MNMB & RMSE \\
\hline TOT & $\mathrm{CE}$ & 1.83 & 1.60 & 0.23 & 14.02 & 1 & 0.94 & 36519 & 0.29 & 0.96 & 1.02 & 14.18 & 0.30 \\
\hline TOT & $\mathrm{CM}$ & 2.11 & 1.91 & 0.20 & 10.42 & 1 & 0.94 & 21573 & 0.28 & 0.96 & 1.03 & 10.42 & 0.28 \\
\hline TOT & $\mathrm{EE}$ & 1.77 & 1.60 & 0.17 & 10.82 & 1 & 0.95 & 40467 & 0.23 & 0.96 & 1.01 & 11.50 & 0.27 \\
\hline TOT & EM & 1.90 & 1.72 & 0.19 & 10.85 & 1 & 0.92 & 52029 & 0.23 & 0.97 & 1.05 & 10.87 & 0.29 \\
\hline TOT & $\mathrm{EU}$ & 1.82 & 1.63 & 0.19 & 11.98 & 1 & 0.95 & 353722 & 0.18 & 1.01 & 1.07 & 11.71 & 0.28 \\
\hline TOT & IP & 1.93 & 1.68 & 0.24 & 14.43 & 1 & 0.93 & 16779 & 0.31 & 0.96 & 1.03 & 14.27 & 0.31 \\
\hline TOT & LA & 1.72 & 1.54 & 0.18 & 11.42 & 1 & 0.95 & 223297 & 0.20 & 0.98 & 1.04 & 11.56 & 0.26 \\
\hline TOT & NA & 1.67 & 1.54 & 0.13 & 8.66 & 1 & 0.94 & 50760 & 0.21 & 0.95 & 1.01 & 8.98 & 0.22 \\
\hline TOT & $\mathrm{NE}$ & 1.56 & 1.38 & 0.18 & 13.05 & 1 & 0.97 & 43381 & 0.03 & 1.11 & 1.14 & 11.80 & 0.25 \\
\hline TOT & $\mathrm{OC}$ & 1.99 & 1.77 & 0.23 & 12.81 & 1 & 0.94 & 130425 & 0.16 & 1.04 & 1.11 & 11.97 & 0.30 \\
\hline
\end{tabular}




\section{Surface Albedo}

a)

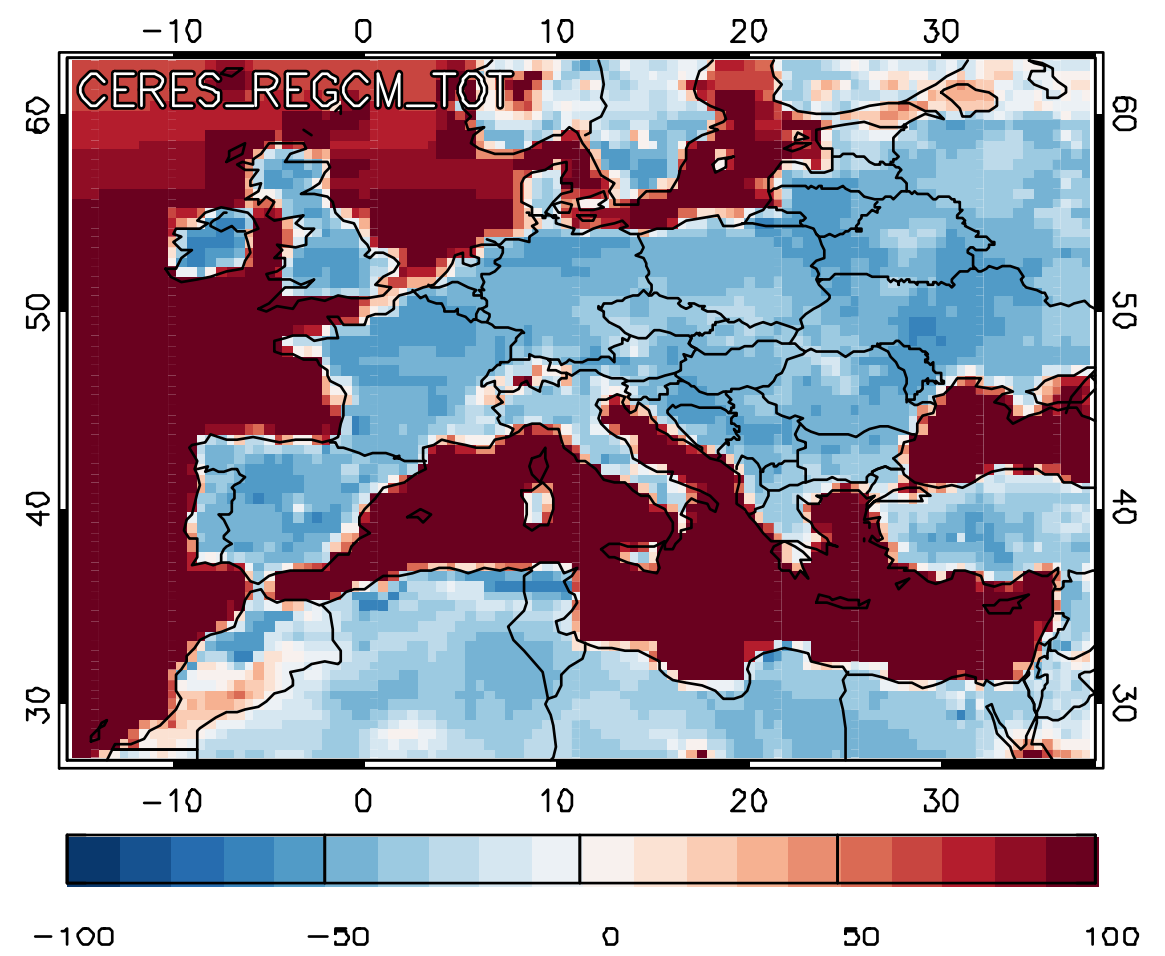

b)

\section{EUROPE_CERES_REGCM_TOT}

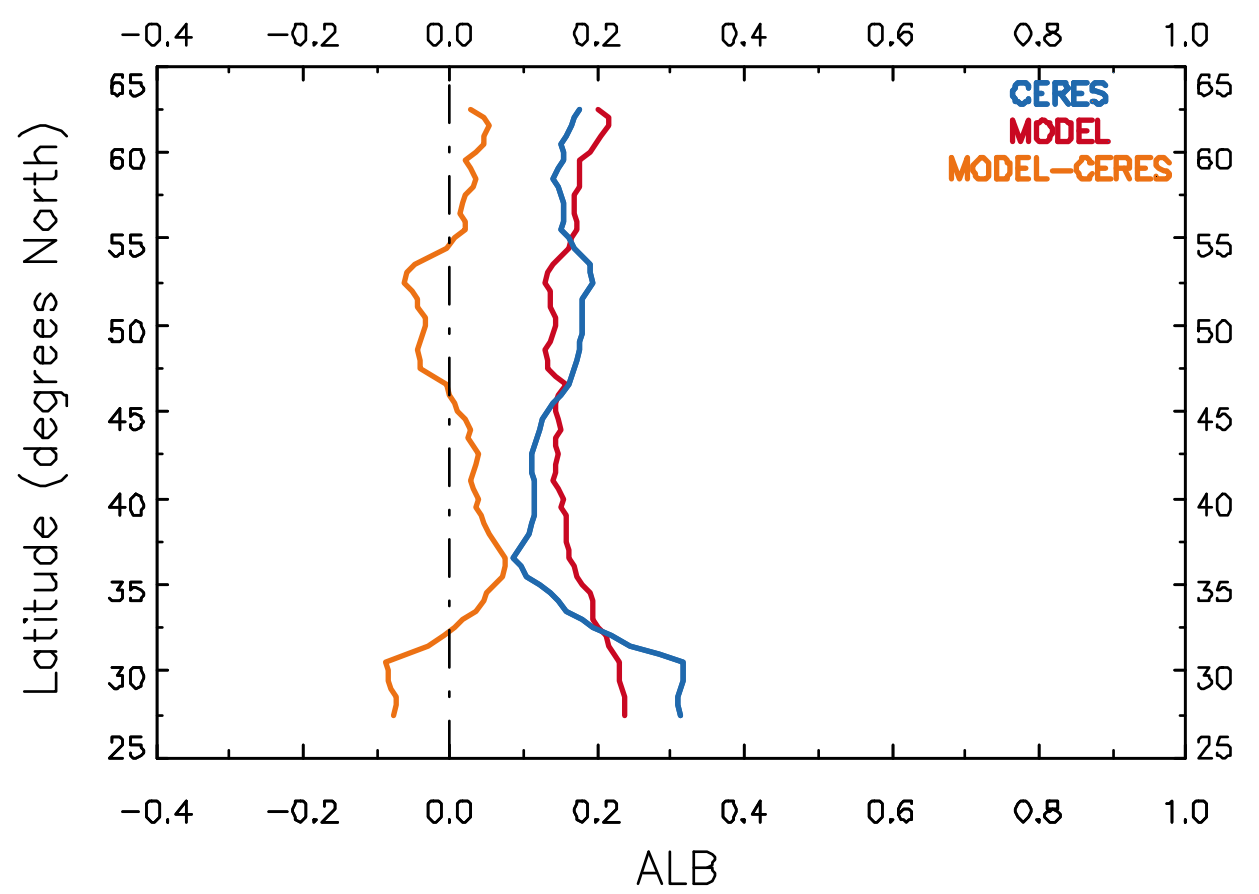

Figure S19. (a) NMB patterns of RegCM4-CERES ALB and (b) Latitudinal variability of RegCM4 ALB (red), CERES ALB (blue) and their difference (orange) over Europe. 
a)

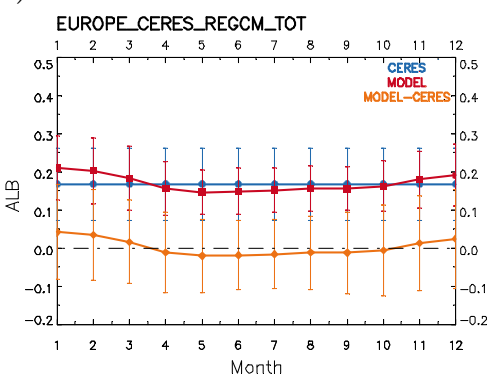

d)

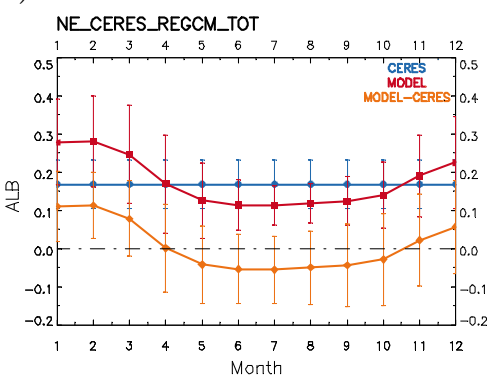

g)

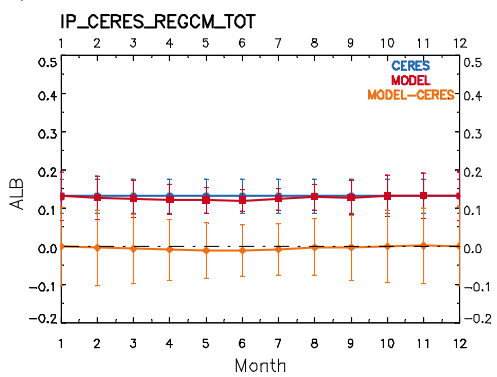

j)

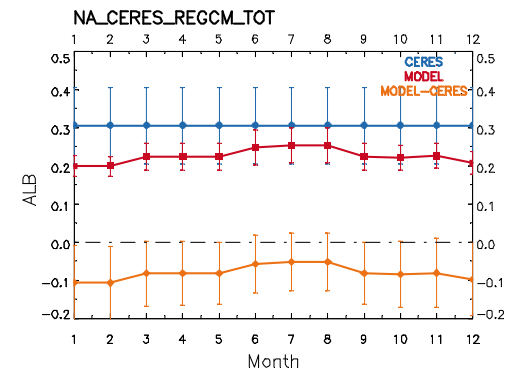

b)

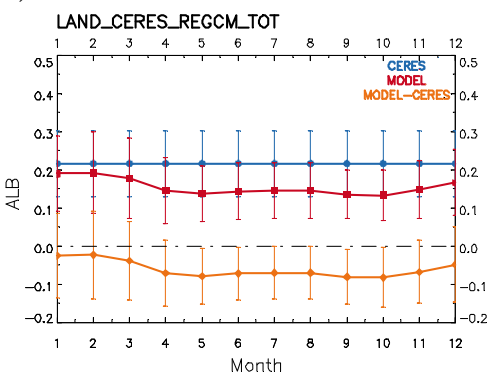

e)

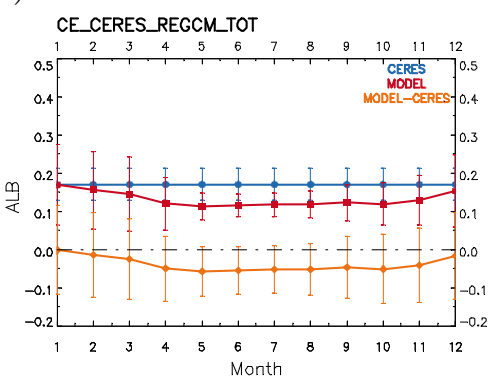

h)

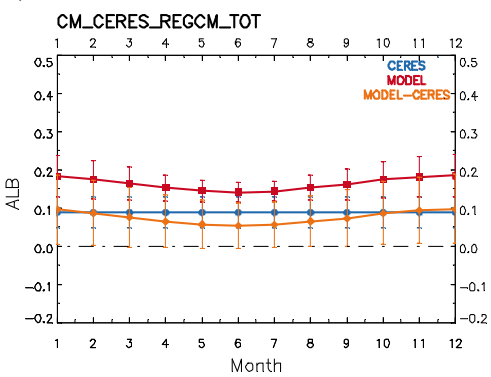

c)

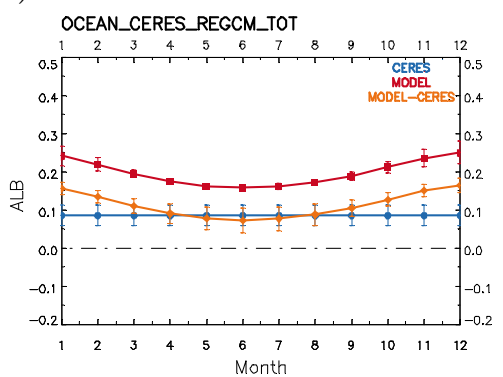

f)

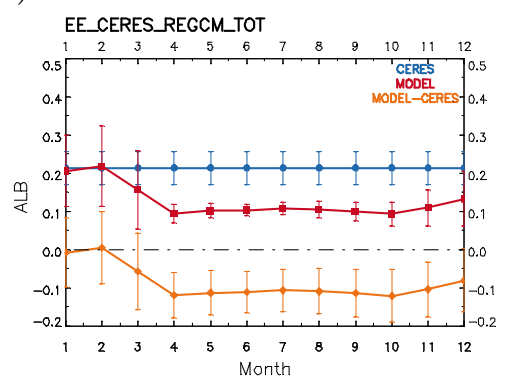

i)

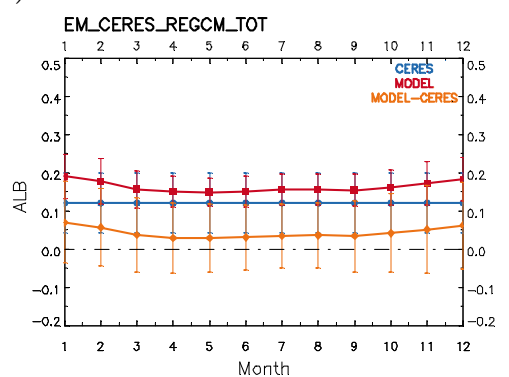

Figure S20. Seasonal variability of RegCM4 ALB (red), CERES ALB (blue) and their difference (orange) over (a) the whole Europe, (b) Land, (c) Ocean, (d) NE, (e) CE, (f) EE, (g) IP, (h) CM, (i) EM, (j) NA. 
Table S14. Average RegCM4 ALB, CERES ALB, their difference, the corresponding NMB and the statistical significance of the results at the $95 \%$ confidence level due to a two independent sample t-test for each region of interest. The NSD, MNMB and the RMSE are also given along with Pearson's correlation coefficient (R), the number of observations used and the coefficients $a$ and $b$ of the regression line $(\operatorname{RegCM} 4=a \cdot C E R E S+b)$. The region are listed in alphabetic order.

\begin{tabular}{|c|c|c|c|c|c|c|c|c|c|c|c|c|c|}
\hline Season & Region & REG & CER & Diff. & NMB & Signif. & $\mathrm{R}$ & Obs. & $\mathrm{b}$ & $\mathrm{a}$ & NSD & MNMB & RMSE \\
\hline TOT & $\mathrm{CE}$ & 0.13 & 0.17 & -0.04 & -22.71 & 1 & -0.26 & 37296 & 0.21 & -0.45 & 1.74 & -30.05 & 0.10 \\
\hline TOT & $\mathrm{CM}$ & 0.16 & 0.09 & 0.08 & 85.92 & 1 & -0.66 & 22032 & 0.23 & -0.73 & 1.11 & 59.75 & 0.11 \\
\hline TOT & $\mathrm{EE}$ & 0.13 & 0.21 & -0.09 & -40.68 & 1 & -0.02 & 41328 & 0.13 & -0.03 & 1.67 & -56.04 & 0.12 \\
\hline TOT & EM & 0.16 & 0.12 & 0.04 & 35.43 & 1 & -0.17 & 53136 & 0.18 & -0.11 & 0.64 & 38.34 & 0.11 \\
\hline TOT & $\mathrm{EU}$ & 0.17 & 0.17 & 0.00 & 1.58 & 1 & 0.13 & 361248 & 0.15 & 0.10 & 0.79 & 7.11 & 0.11 \\
\hline TOT & IP & 0.13 & 0.13 & 0.00 & -3.76 & 1 & -0.78 & 17136 & 0.24 & -0.84 & 1.08 & -4.75 & 0.09 \\
\hline TOT & LA & 0.15 & 0.22 & -0.06 & -28.27 & 1 & 0.46 & 223297 & 0.06 & 0.46 & 1.00 & -35.62 & 0.11 \\
\hline TOT & NA & 0.23 & 0.31 & -0.08 & -26.38 & 1 & 0.49 & 51840 & 0.16 & 0.20 & 0.40 & -24.21 & 0.12 \\
\hline TOT & $\mathrm{NE}$ & 0.18 & 0.17 & 0.01 & 5.24 & 1 & 0.20 & 44304 & 0.12 & 0.36 & 1.82 & -4.34 & 0.12 \\
\hline TOT & $\mathrm{OC}$ & 0.20 & 0.08 & 0.11 & 131.07 & 1 & 0.21 & 130425 & 0.17 & 0.27 & 1.30 & 80.29 & 0.12 \\
\hline
\end{tabular}




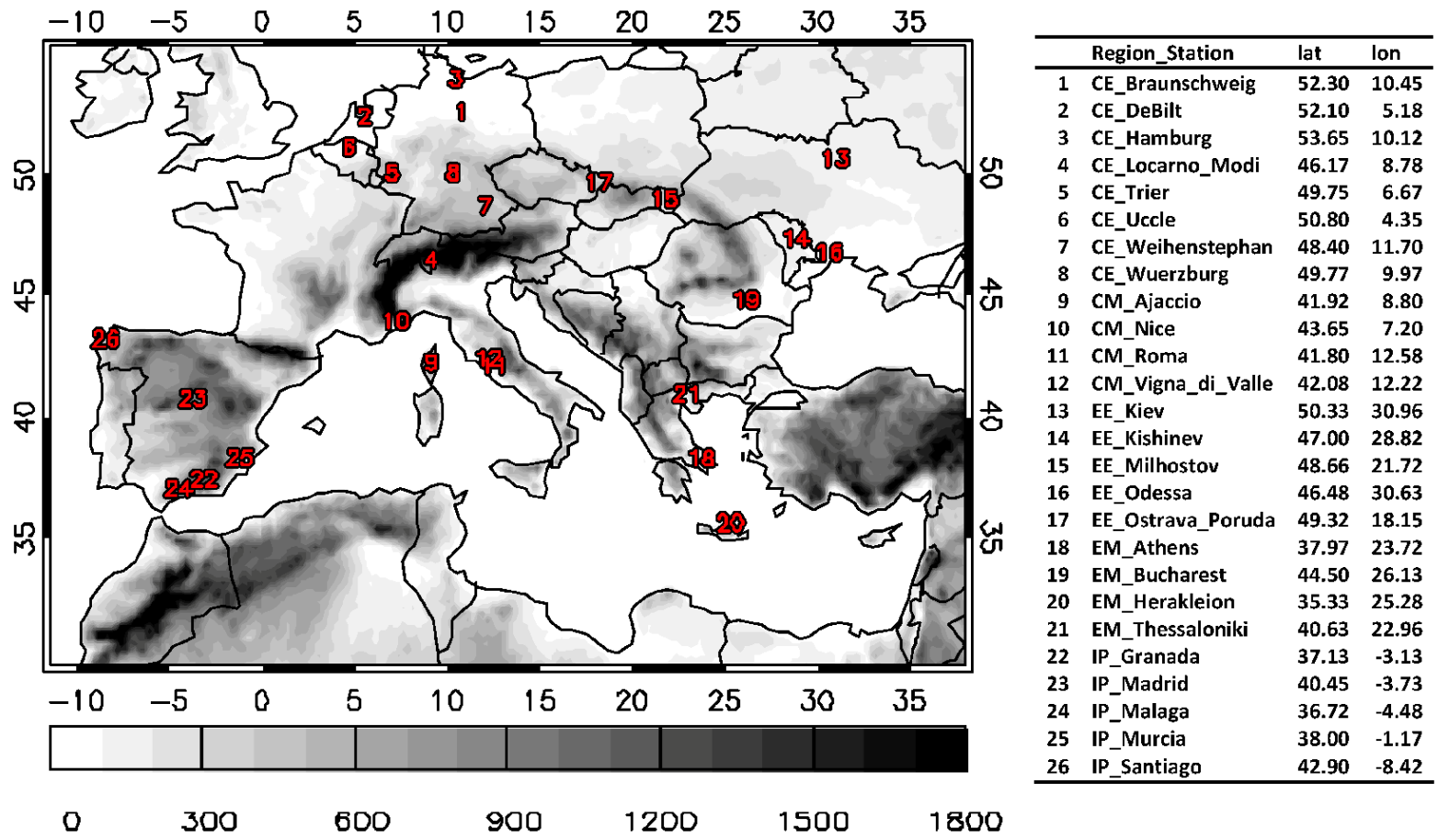

Figure S21. Elevation map (in meters) with the position of the 23 WRDC and 3 independent ground stations which are used for the evaluation of the CM SAF SSR data. The number and the name of each station along with the region where they belong and the exact geolocation are also given. 


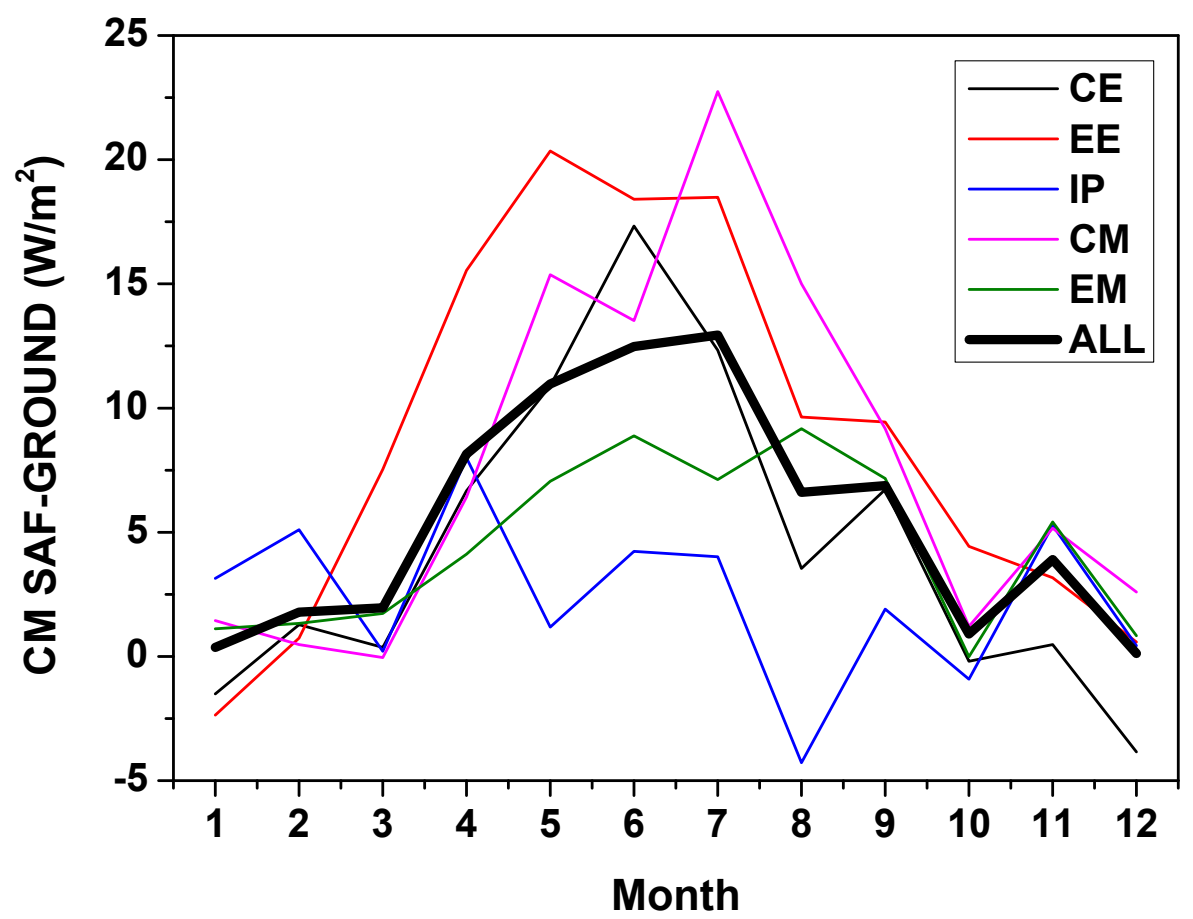

Figure S22. Mean bias between the CM SAF SSR data from MSG and ground-based observations for the period 2006-2009 for the five sub-regions appearing in Fig. 10. 

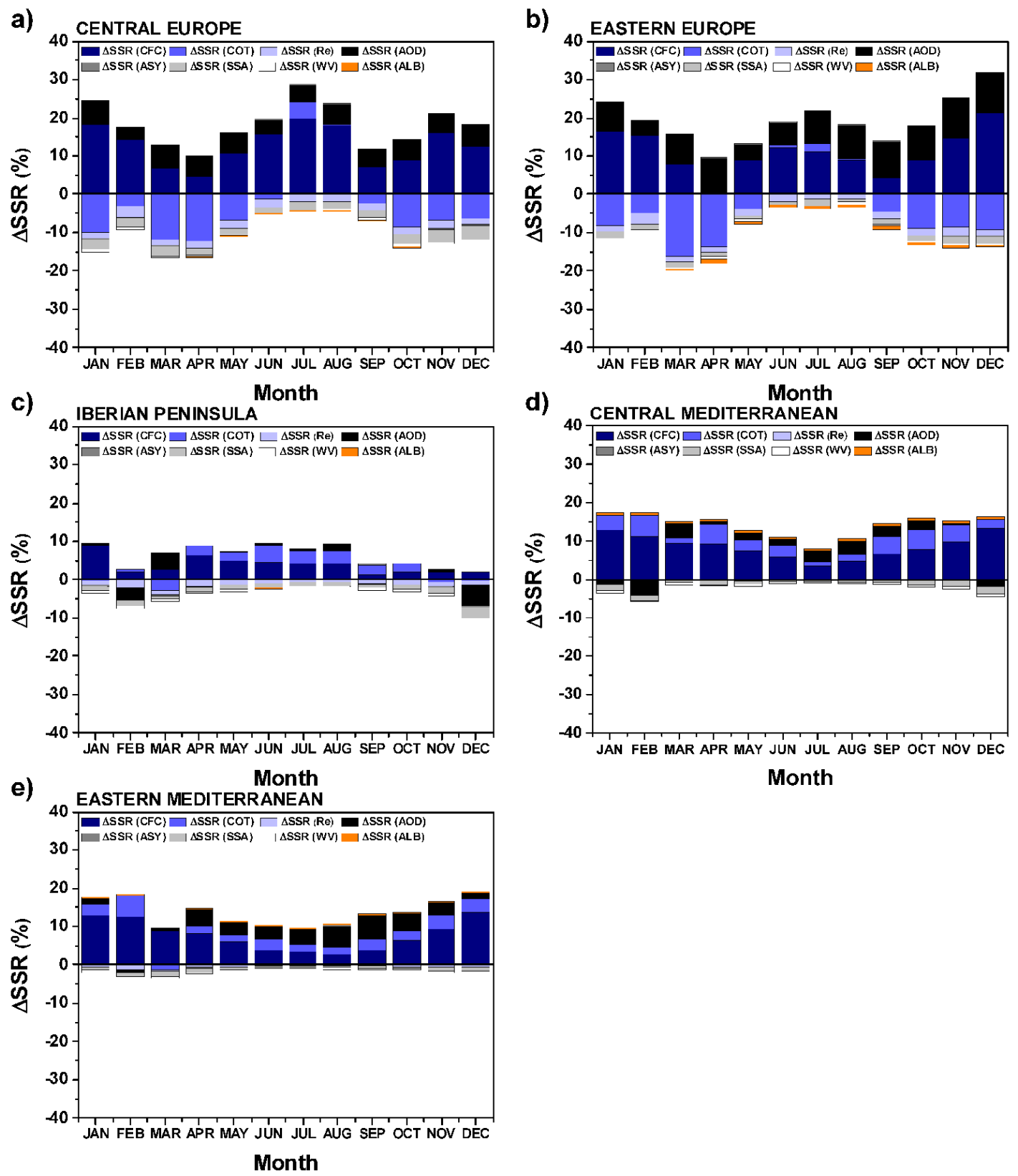
Month

Figure S23. SSR (\%) caused by CFC, COT, Re, AOD, ASY, SSA, WV and ALB for (a) CE, (b) EE, (c) IP, (d) CM and (e) EM. These results were produced assuming the simulated SSR fields with all the CM SAF, MACv1 and ERA-Interim input data as the control run and replacing each time the corresponding parameter with data from RegCM4 (inverse procedure from the one followed for the production of Fig. 10). 
a) 40 CENTRAL EUROPE

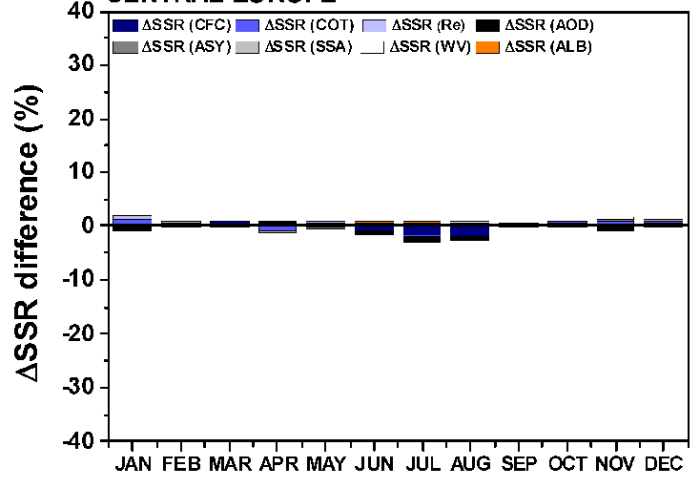

c)

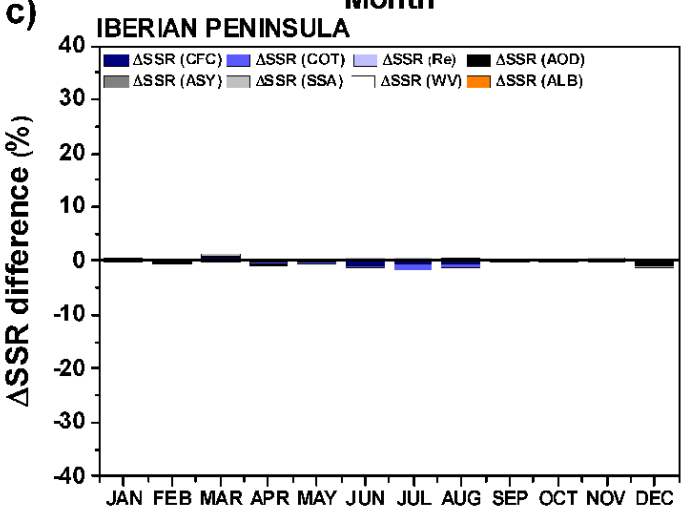

e) Month

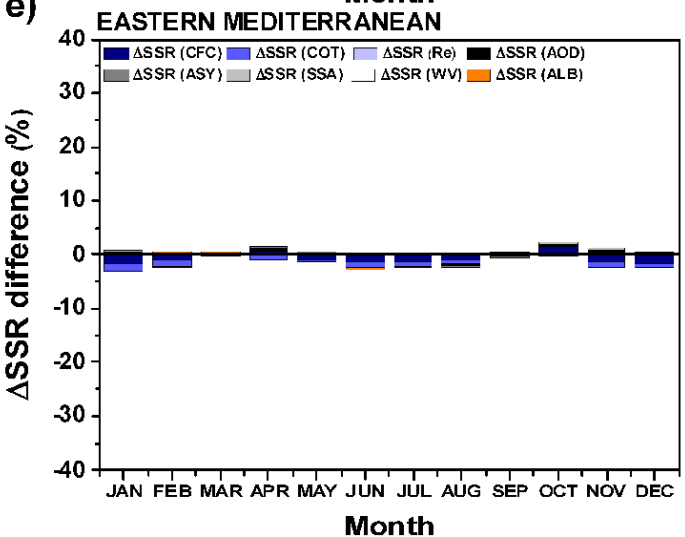

b) 40 EASTERN EUROPE

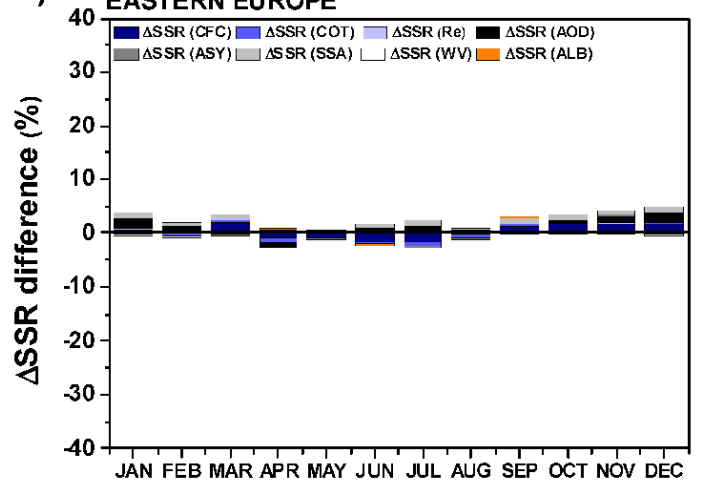

d)

Month

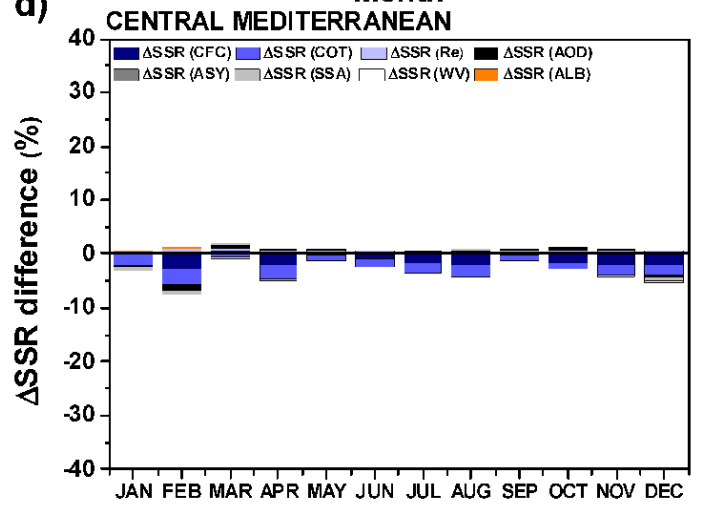

Month

Figure S24. Difference between the results presented in Fig. 10 and Fig. S21. 